Florida International University FIU Digital Commons

9-12-1996

\title{
Theoretical analysis of cross joint geometries and their classification
}

Taixu Bai

Florida International University

DOI: $10.25148 /$ etd.FI14050407

Follow this and additional works at: https://digitalcommons.fiu.edu/etd

Part of the Geology Commons

\section{Recommended Citation}

Bai, Taixu, "Theoretical analysis of cross joint geometries and their classification" (1996). FIU Electronic Theses and Dissertations. 1370. https://digitalcommons.fiu.edu/etd/1370

This work is brought to you for free and open access by the University Graduate School at FIU Digital Commons. It has been accepted for inclusion in FIU Electronic Theses and Dissertations by an authorized administrator of FIU Digital Commons. For more information, please contact dcc@fiu.edu. 


\title{
FLORIDA INTERNATIONAL UNIVERSITY
}

\author{
Miami, Florida
}

\section{THEORETICAL ANALYSIS OF CROSS JOINT GEOMETRIES AND THEIR CLASSIFICATION}

\begin{abstract}
A thesis submitted in partial satisfaction of the requirements for the degree of MASTER OF SCIENCE IN

GEOLOGY by
\end{abstract}

Taixu Bai 
To: Dean Arthur W. Herriott

College of Arts and Sciences

This thesis, written by Taixu Bai, and entitled THEORETICAL

ANALYSIS OF CROSS JOINT GEOMETRIES AND THEIR

CLASSIFICATION, having been approved in respect to style and intellectual content, is referred to you for judgment.

We have read this thesis and recommend that it be approved.

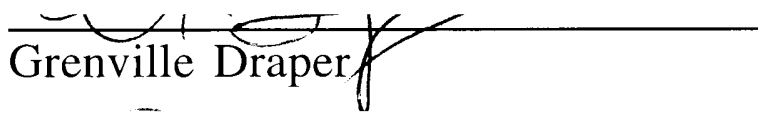

Dean Whitman

Míchael R. Gross, Major Professor

Date of Defense: September 12, 1996

The thesis of Taixu Bai is approved.

Dean Arthur W. Herriott

College of Arts and Sciences

Dr. Richard L. Campbdll

Dean of Graduate Studies

Florida International University, 1996 
드 COPYRIGHT 1996 by Taixu Bai

All rights reserved 
To my parents 


\section{ACKNOWLEDGMENTS}

I would like to express my deepest appreciation to my advisor, Dr. Michael R. Gross, for all his help during my two years at Florida International University. With his advisement and support, I greatly enjoyed my experiences at FIU and the city of Miami.

I wish to thank the other two members of my committee, Dr. Grenville Draper and Dr. Dean Whitman, for their helpful comments, advisement and patience in reading this thesis. I wish to thank our laboratory managers Ms. Lois Geier and Ms. Diane Pirie for their help in improving my teaching skills and research. Also, I wish to thank all of the professors, engineers, secretaries and all of my fellow graduate students for creating such a nice academic environment in our department.

I have greatly benefited from discussions with Dr. Genady Cherepanov, involving both my coursework and research. I feel fortunate to have been associated with such a nice and knowledgeable professor in my life. I thank Dr. Alexander Becker for providing me with the cross joint examples from Israel.

Finally, I would like to thank my wife Jie Liu for her support and encouragement. She joined me at the expense of losing her faculty position at the Beijing Institute of Technology. I am greatly indebted to my daughter Yanran Bai, who is still living with her grandmother in China.

This research was partially supported by the U.S.-Israel Binational Science Foundation grant No. 94-00396. 


\title{
ABSTRACT OF THE THESIS
}

\section{THEORETICAL ANALYSIS OF CROSS JOINT GEOMETRIES AND THEIR CLASSIFICATION}

\author{
by \\ Taixu Bai \\ Florida International University, 1996 \\ Miami, Florida \\ Professor Michael R. Gross, Major Professor
}

Joints as opening-mode fractures play important roles as indicators of tectonic stress fields and as pathways for underground fluid flow. This thesis analytically investigates the relationships among cross joint geometry, orientations and ratios of remote principal stresses, and fluid pressure. Results show that main trends of cross joints are perpendicular to the least far field stresses during cross joint formation, and cross joint paths can be used to determine relative magnitudes of remote principal stresses. Based on the theoretical derivation, cross joint geometries are grouped into five main categories: curving-parallel, curving-perpendicular, quasi-curving-parallel, quasi-curving-perpendicular and non-curving geometries. By introducing the concepts of effective stress and effective remote principal stress ratio, it is demonstrated that connectivity between cross joints and the pre-existing joint is improved for joints that form under relatively high pore pressures. 


\section{TABLE OF CONTENTS}

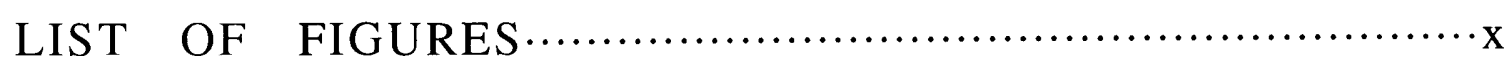

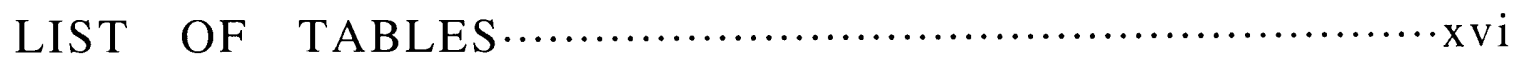

LIST OF SYMBOLS………......................................

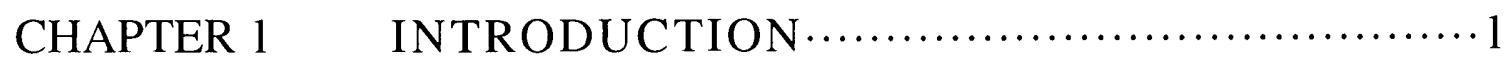

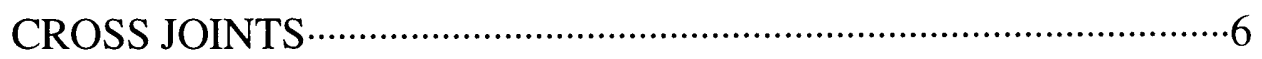

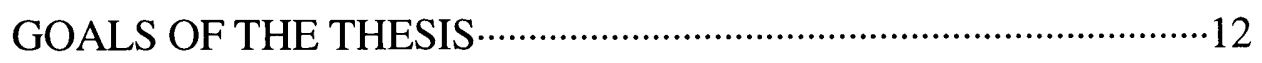

CHAPTER 2 DYER'S (1988) ANALYSIS ….........................14

CHAPTER 3 THEORETICAL DERIVATION….....................25

COORDINATE SYSTEM AND ASSUMPTIONS IN THIS STUDY ………..26 CONDITIONS FOR OPEN AND CLOSED PRE-EXISTING JOINTS ……..30 CROSS JOINT GEOMETRY AS A FUNCTION OF REMOTE PRINCIPAL

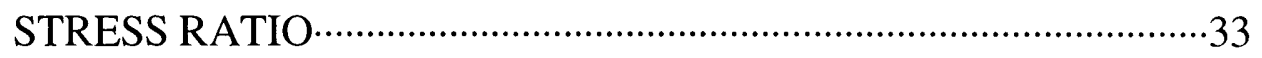

I. OPEN PRE-EXISTING JOINT CASE ……………………………....33

(1). Conditions for curving-parallel and curvingperpendicular geometries.……………………………..33

(2). Local stresses adjacent to the pre-existing joint ………...37

(3). Cross joint trajectories ……………………………………....40

II. CLOSED PRE-EXISTING JOINT CASE …………………………….....42

(1). Local stresses adjacent to the pre-existing joint ………...47

(2). General formulation of local cross joint angle and cross joint geometry classification. 
(3). Cross joint geometry and the coefficient of friction along pre-existing joint_—detailed cross joint geometry classification

COMPRESSIVE ZONE: CONSTRAINTS ON CROSS JOINT

GROWTH

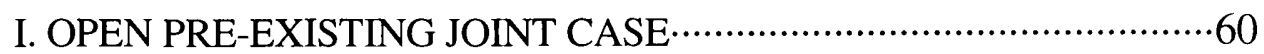

II. CLOSED PRE-EXISTING JOINT CASE………................................64

INFLUENCES OF PORE PRESSURE ON CROSS JOINT GROWTH $\cdots \cdots \cdots . .70$

I. EFFECTIVE STRESS AND EFFECTIVE REMOTE PRINCIPAL

STRESS RATIO

II. CROSS JOINT GEOMETRY AND PORE PRESSURE .76

SUMMARY

\section{CHAPTER 4}

APPLICATION OF CROSS JOINT THEORY TO

FIELD EXAMPLES

INTRODUCTION

PROCEDURES FOR FIELD DATA COLLECTION AND ANALYSIS $\cdots .88$

EXAMPLE 1: CURVING PARALLEL AND CURVING-

PERPENDICULAR CROSS JOINTS FROM THE

MONTEREY FORMATION, CALIFORNIA

I. CROSS JOINT ANALYSIS.

II. INTERPRETATION OF CROSS JOINTS IN THE MONTEREY FORMATION

EXAMPLE 2: A QUASI-CURVING-PARALLEL CROSS

JOINT FROM THE GEROFIT FORMATION, ISRAEL 108 
EXAMPLE 3: NON-CURVING AND CURVING PERPENDICULAR

CROSS JOINTS FROM BELMONT, NEW YORK

SUMMARY

CHAPTER 5 DISCUSSION AND SUMMARY $\cdots \cdots \cdots \cdots \cdots \cdots \cdots \cdots \cdots \cdots \cdots$

CROSS JOINT GEOMETRY AND FLUID CONDUCTIVITY ……………125

SIGNIFICANCE OF COMPRESSIVE ZONE ……....................................129

SIGNIFICANCE OF THE RELATION BETWEEN CROSS JOINT

GEOMETRY AND FLUID PRESSURE..........................................1 131

LIMITATIONS IN USING THE THEORETICAL RESULTS ……………....132

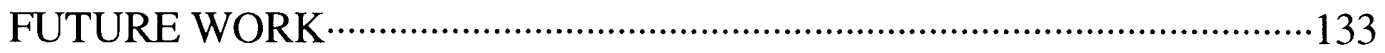

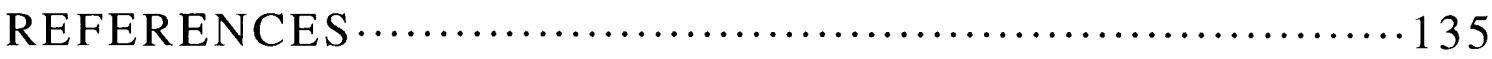

APPENDIX A HOW TO CONSTRUCT A THEORETICAL CROSS

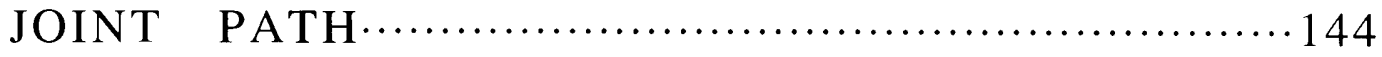

VITA 


\section{LIST OF FIGURES}

Fig. 1-1. Principal stress configuration during joint growth …...........2

Fig. 1-2. Regional joint pattern on the Appalachian Plateau …..............4

Fig. 1-3. (a) Dike patterns at Spanish Peaks, Colorado. (b) Theoretical regional stress field at Spanish Peaks, Colorado ……………......5

Fig. 1-4. Some cross joint examples from the literature.....................

Fig. 1-5. Schematic diagram showing systematic pre-existing joints and cross joints observed by Dyer (1988)...............................9

Fig. 1-6. Several of the joint-system architectural styles on bedding planes described by Hancock (1985) …..................................11

Fig. 2-1. Dyer's conceptual model …….............................. 15

Fig. 2-2. Dyer's stress configuration and coordinate system ………....16

Fig. 2-3. The four cross joint subclasses of Dyer (1988) ……............19

Fig. 2-4. Dyer's curving-parallel and curving-perpendicular cases for an

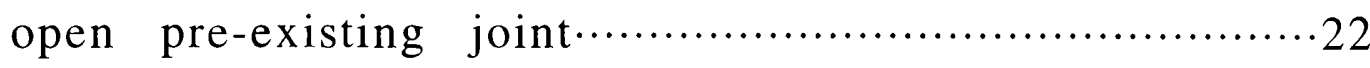

Fig. 2-5. Dyer's frictional and frictionless cases for a closed pre-existing joint

Fig. 3-1. Coordinate system and the stress state in forming the pre-existing joint used in this study 
Fig. 3-2. Formation of cross joint after rotation of the horizontal remote principal stresses

Fig. 3-3. Tripolar coordinate system used in deriving local stresses …..38

Fig. 3-4. Schematic diagram showing the definitions of take off angle $(\varsigma)$

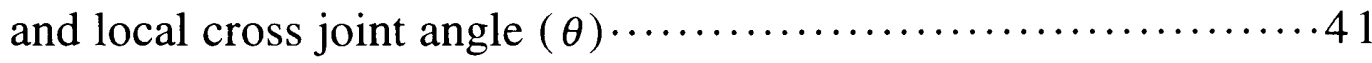

Fig. 3-5. Relationships between local cross joint angle ( $\theta)$ and the preexisting joint) and the normalized distance from the pre-existing joint $(y / c)$ for different remote principal stress ratios $(\eta)$, a take off angle $(\varsigma)$ of $30^{\circ}$, and under the conditions for an open pre-existing joint

Fig. 3-6. Physical views of cross joint propagation paths corresponding to Fig.

Fig. 3-7. Relationships between local cross joint angle $(\theta)$ and the normalized distance from the pre-existing joint $(y / c)$ for different remote principal stress ratios $(\eta)$, a take off angle $(\varsigma)$ of $60^{\circ}$, and under the conditions for an open pre-existing joint

Fig. 3-8. Physical views of cross joint propagation paths corresponding to Fig. 3-7. The takeoff angle $(\varsigma)$ is $60^{\circ}$

Fig. 3-9. Relationships between local cross joint angle $(\theta)$ and the normalized distance from the pre-existing joint $(y / c)$ for different remote principal stress ratios $(\eta)$, a take off angle $(\varsigma)$ of $30^{\circ}$, and under the conditions for a closed pre-existing joint 
Fig. 3-10. Physical views of cross joint propagation paths corresponding

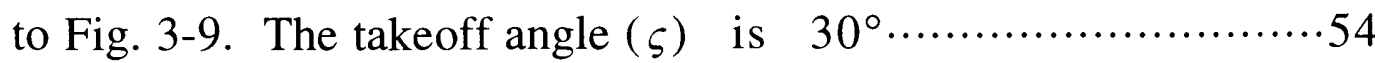

Fig. 3-11. Relations between the critical value of the coefficient of friction $\left(C_{0}^{*}\right)$ along the pre-existing joint and the remote principal stress ratio

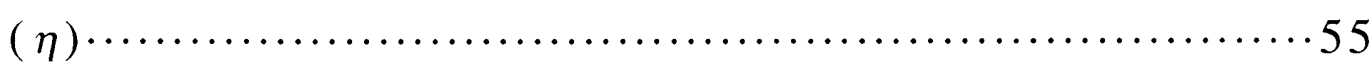

Fig. 3-12. Relationships between local cross joint angle $(\theta)$ and the normalized distance from the pre-existing joint $(y / c)$ for different remote principal stress ratios $(\eta)$, a take off angle $(\varsigma)$ of $60^{\circ}$, and under the conditions for a closed pre-existing joint using a coefficient of friction along the pre-existing joint of $0.6 \cdots \cdots \cdots \cdots . . .57$

Fig. 3-13. Physical views of cross joint propagation paths corresponding

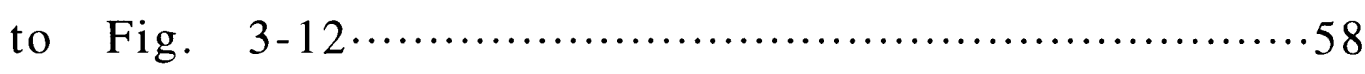

Fig. 3-14. Cross joint geometry classification based on takeoff angle ( $\varsigma)$ and remote principal stress ratio $(\eta)$

Fig. 3-15. (a) Dimension of compressive zone $\left(t_{0}\right)$ as a function of remote principal stress ratio $(\eta)$

Fig. 3-16. Cross joint geometries and their characteristics as a function of takeoff angle $(\varsigma)$ and remote principal stress ratio $(\eta) \ldots \ldots \ldots \ldots . . .71$

Fig. 3-17. (a) Relationships between the effective remote principal stress ratio $\left(\eta^{*}\right)$ and the remote principal stress ratio $(\eta)$.(b) Relationships between the effective remote principal stress ratio $\left(\eta^{*}\right)$ and the pore pressure to the least principal stress ratio $\left(P_{p} / \sigma_{3}^{\infty}\right) \ldots \ldots \ldots \ldots \ldots . \ldots \ldots$ 
Fig. 3-18. Cross joint geometry variation as a function of pore pressure....

Fig. 3-19. Cross joint geometry variation as a function of pore

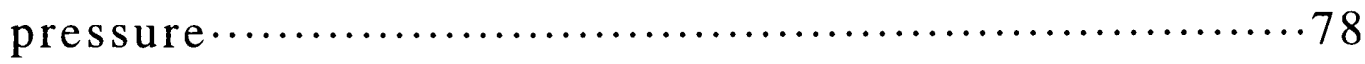

Fig. 3-20. Cross joint geometry variation as a function of pore pressure....

Fig. 3-21. Cross joint geometry variation as a function of pore pressure....

Fig. 3-22. Cross joint geometry variation as a function of pore pressure....

Fig. 4-1. Procedures for applying the analytical solutions to cross joints observed in the field

Fig. 4-2. Regional structural map of the western Transverse Ranges.....93

Fig. 4-3. (a) A photo of cross joint examples from the Monterey formation, California. (b) Sketch of the pre-existing joint and cross

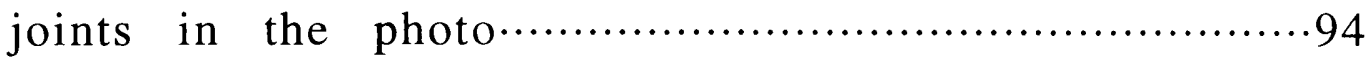

Fig. 4-4. (a) Calculated cross joint paths for different remote principal stress ratios with a takeoff angle of $39^{\circ}$. (b) Comparison of observed and calculated cross joint paths ……..............................96

Fig. 4-5. (a) Calculated cross joint paths for different remote principal stress ratios with a takeoff angle of $34^{\circ}$. (b) Comparison of observed and calculated cross joint paths....................................97 
Fig. 4-6. (a) Calculated cross joint paths for different remote principal stress ratios with a takeoff angle of $37^{\circ}$. (b) Comparison of observed and calculated cross joint paths......................................98

Fig. 4-7. (a) Calculated cross joint paths for different remote principal stress ratios with a takeoff angle of $34^{\circ}$. (b) Comparison of observed and calculated cross joint paths ………...........................99

Fig. 4-8. (a) Plot of effective remote principal stress ratio $\left(\eta^{*}\right)$ versus fluid pressure/least principal stress ratio $\left(\mathrm{P}_{\mathrm{p}} / \sigma_{3}^{\infty}\right)$ at a constant remote principal stress ratio $(\eta)$. (b) Mohr circle diagram showing stress state variation as a function of fluid pressure $\left(\mathrm{P}_{\mathrm{p}}\right)$ with constant remote principal stresses $\left(\sigma_{2}^{\infty}\right.$ and $\sigma_{3}^{\infty}$ ). (c) Mohr circle diagram showing stress state variation as a function of maximum horizontal stress $\left(\sigma_{2}^{\infty}\right)$ with constant minimum horizontal stress $\left(\sigma_{3}^{\infty}\right)$ and fluid

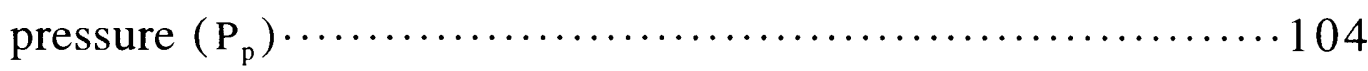

Fig. 4-9. (a) Block diagram of a joint surface showing arrest lines, plume axis and fracture propagation direction. (b) Fluid pressure variation through time during incremental joint propagation..................106

Fig. 4-10. (a) A photo of cross joint example from Israel. (b) Sketch of the pre-existing joint and the cross joint in the photo ………......109

Fig. 4-11. A simplified structural map of Southern Israel showing the locations of Zin Fault and Haluza Anticline 
Fig. 4-12. Plots of dimension of compression versus remote effective principal stress ratio for Closed Case \#1 (Table 2-3) with a take off angle of $28^{\circ}$

Fig. 4-13. (a) Calculated cross joint paths for different remote principal stress ratios with a takeoff angle of $28^{\circ}$. (b) Comparison of observed and calculated cross joint paths ………....................... 114

Fig. 4-14. (a) Map showing the location of Belmont, New York. (b) Sketch diagram showing the three joint sets in Canadaway Group siltstones in Genesee River bed at Belmont, New York

Fig. 4-15. Relation between the maximum value of remote effective principal stress ratio and the coefficient of friction along the preexisting joint for the non-curving cross joint, i.e., cross joint (3b), in Fig. $\quad 4-10$

Fig. 5-1. Sketch diagrams showing the relationships between cross joint geometries and fluid conductivity of rocks

Fig. 5-2. Cross joint geometries and their relative lengths of their paths 


\section{LIST OF TABLES}

Table 3-1. Conditions for open and closed pre-existing joints ……......32

Table 3-2. Classification of cross joint geometries............................36

Table 3-3. Summary of cross joint geometries and their characteristics $* 63$

Table 4-1. Principal stress ratios estimated from the cross joint examples in Fig. $4-1$

Table 4-2. Remote effective principal stress and pre-existing joint

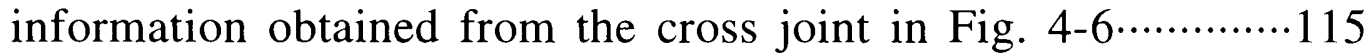

Table 4-3. Remote effective principal stress and pre-existing joint information obtained from cross joint (3a) and (3b) in Fig. 


\section{LIST OF SYMBOLS}

$c$

$r$

$r_{1}$

$r_{2}$

$S$

$t$

$t_{0}$

$x, y, z$,

$C_{0}$

$C_{0}^{*}$

K

$K_{i}$

$K_{j}$

$K_{s}$

$P_{p}$

$R$

$\alpha$

$\delta_{i j}$ half height of a pre-existing joint or a cross joint distance from the center of the pre-existing joint to a point in the $x y$-plane, $r=\sqrt{x^{2}+y^{2}}$

distance from the left end of the pre-existing joint (Fig.

2-14) to a point in the $x y$-plane, $r_{1}=\sqrt{(x+c)^{2}+y^{2}}$

distance from the right end of the pre-existing joint

(Fig. 2-14) to a point in the $x y$-plane, $r_{2}=\sqrt{(x-c)^{2}+y^{2}}$

shear stress ratio defined by $s=\sigma_{\mathrm{yz}}(x=0) / \sigma_{\mathrm{vz}}^{\infty}$

normalized distance from the pre-existing joint, $y / c$

normalized dimension of compressive zone

axes and coordinates with $x$-axis is vertical and parallel

to the pre-existing joint, $y$-axis is horizontal and

perpendicular to the pre-existing joint, and $z$-axis is

horizontal and parallel to the pre-existing joint

coefficient of friction

critical value of coefficient of friction

bulk modulus of saturated rock

fluid conductivity in $i$ direction

fluid conductivity in $j$ direction

intrinsic modulus of rock material

fluid pressure

defined as $R=\sqrt{r_{1} r_{2}}$

Biot pore-pressure coefficient

Kroenecker's delta 
$\psi_{1}$

$\psi_{2}$

take-off angle of a cross joint

far field principal stress ratio, $\sigma_{2}^{\infty} / \sigma_{3}^{\infty}$

far field effective principal stress

local cross joint angle

Poisson ratio

local maximum principal stress

local intermediate principal stress

local minimum principal stress

far field maximum principal stress

far field intermediate principal stress

far field minimum principal stress

local normal stress in $i(i=x, y, z)$ direction

local shear stress in $j(j=x, y, z)$ direction and acting on

a plane normal to $i(i=x, y, z)$ direction

far field normal stress in $i(i=x, y, z)$ direction

far field shear stress in $i(i=x, y, z)$ direction and acting

on a plane normal to $j(j=x, y, z)$ direction

effective stress

angle between a line from the center of the pre-existing joint to a point in the $x y$-plane and the $x$-axis

angle between a line from the left end of the pre-

existing joint (Fig. 2-14) to a point in the $x y$-plane and the $x$-axis

angle between a line from the right end of the preexisting joint (Fig. 2-14) to a point in the $x y$-plane and the $x$-axis 


\section{Chap?e⿻上丨}

\section{\{}

\section{INTRODUCTION}

The general term "fracture" refers to any planar to curvi-planar discontinuity found in rock, and includes a wide variety of features such as joints, faults, shear fractures, and veins. Although fracture type depends upon applied forces, mechanical properties, sense of displacement, and fluid chemistry, all fractures are manifestations of brittle deformation. Brittle deformation mechanisms operate under relatively low temperatures and differential stresses (e.g., Twiss and Moores, 1992), and thus fractures most commonly develop in the earth's upper crust (upper 10-15 km).

"Joints" are unmineralized fractures that display no appreciable shear displacement. Because displacement is normal to the fracture plane, joints are referred to as "opening-mode", or "mode-I" fractures in the fracture mechanics literature (Engelder, 1987; Pollard and Aydin, 1988). Joints always propagate normal to the local least principal stress $\left(\sigma_{3}\right)$ and in the plane containing maximum $\left(\sigma_{1}\right)$ and intermediate $\left(\sigma_{2}\right)$ principal stresses 


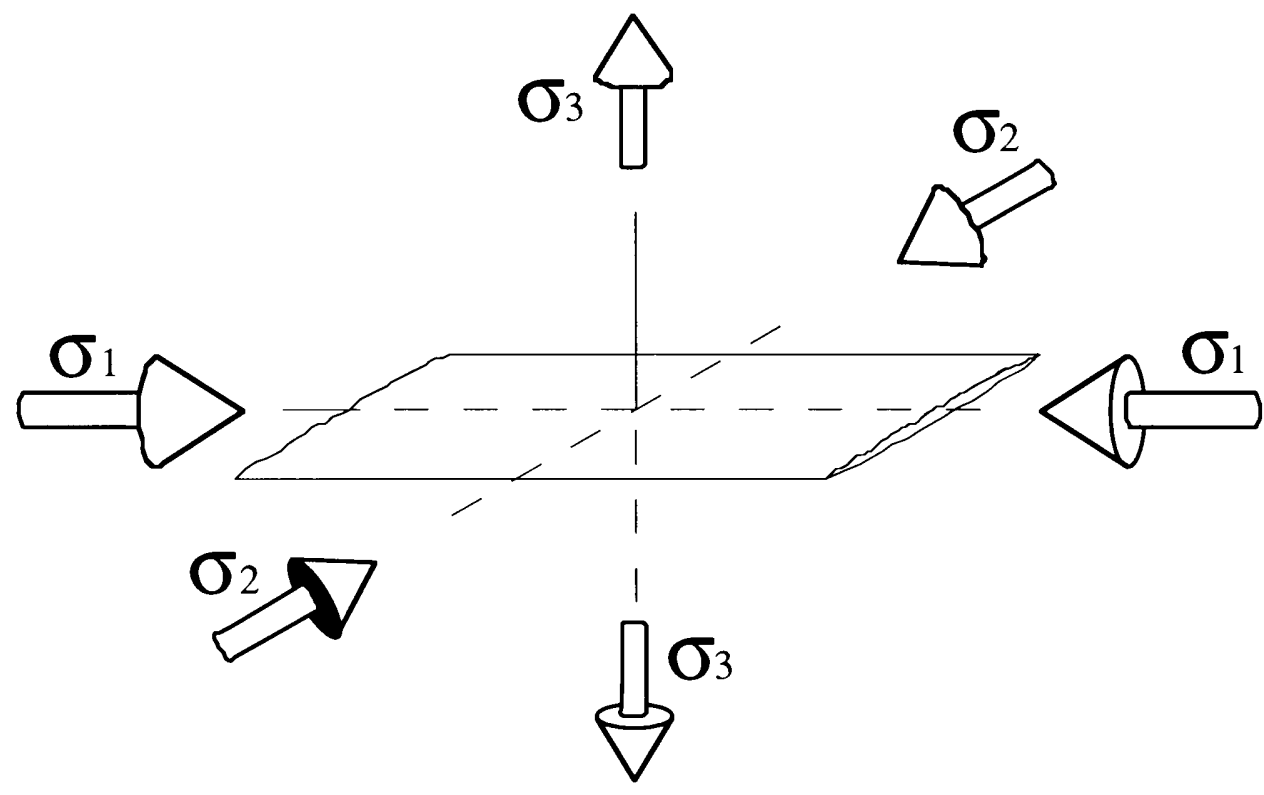

Fig. 1-1. Principal stress configuration during joint growth. Joints as mode-I fractures always propagate perpendicular to the least principal stress $\left(\sigma_{3}\right)$ and in the plane containing the maximum $\left(\sigma_{1}\right)$ and intermediate principal stress $\left(\sigma_{2}\right)$. The sign convention of positive for compressive stresses is used in this figure and throughout the thesis. 
(Fig. 1-1). Consequently, systematic changes in joint orientation may reflect changes in the local stress field near the tip of a propagating joint. Therefore, joints can be used as sensitive indicators of both local and regional stress fields at the time of brittle failure.

Two examples of how mode-I fractures describe past tectonic stress fields are shown in Figs. 1-2 and 1-3. The arcuate pattern of regional joint orientations on the Appalachian Plateau of southern New York and western Pennsylvania may be used to construct trajectories of maximum horizontal stress $\left(S_{H}\right)$ during the Alleghenian orogeny (Engelder and Geiser, 1980). As expected, the stress trajectories trend toward the hinterland of the orogen (Fig. 1-2). A second example of mode-I fractures serving as indicators of stress orientation is found in dike patterns at Spanish Peaks, Colorado (Odé 1957; Johnson, 1968; Muller and Pollard, 1977). Dike trends reflect a combination of local stresses resulting from igneous intrusion in addition to a regional ENE tectonic compressive stress. As shown in Fig. 1-3, theoretical stress trajectories computed by superimposing stresses around a pressurized hole (simulating an igneous intrusion) onto a rectilinear stress field (simulating regional tectonic stress) compare favorably with the observed dike pattern.

Mode-I fractures have also been used to estimate stress magnitude during brittle deformation. For example, Segall and Pollard (1983) calculated the minimum principal stress and fluid pressure required to drive a set of opening-mode fractures in granitic rock based on vein apertures and fracture mechanics theory. By analyzing the fluid inclusion geochemistry of opening-mode veins, Srivastava and Engelder (1990) were able to constrain fluid pressures and local states of stress during progressive 


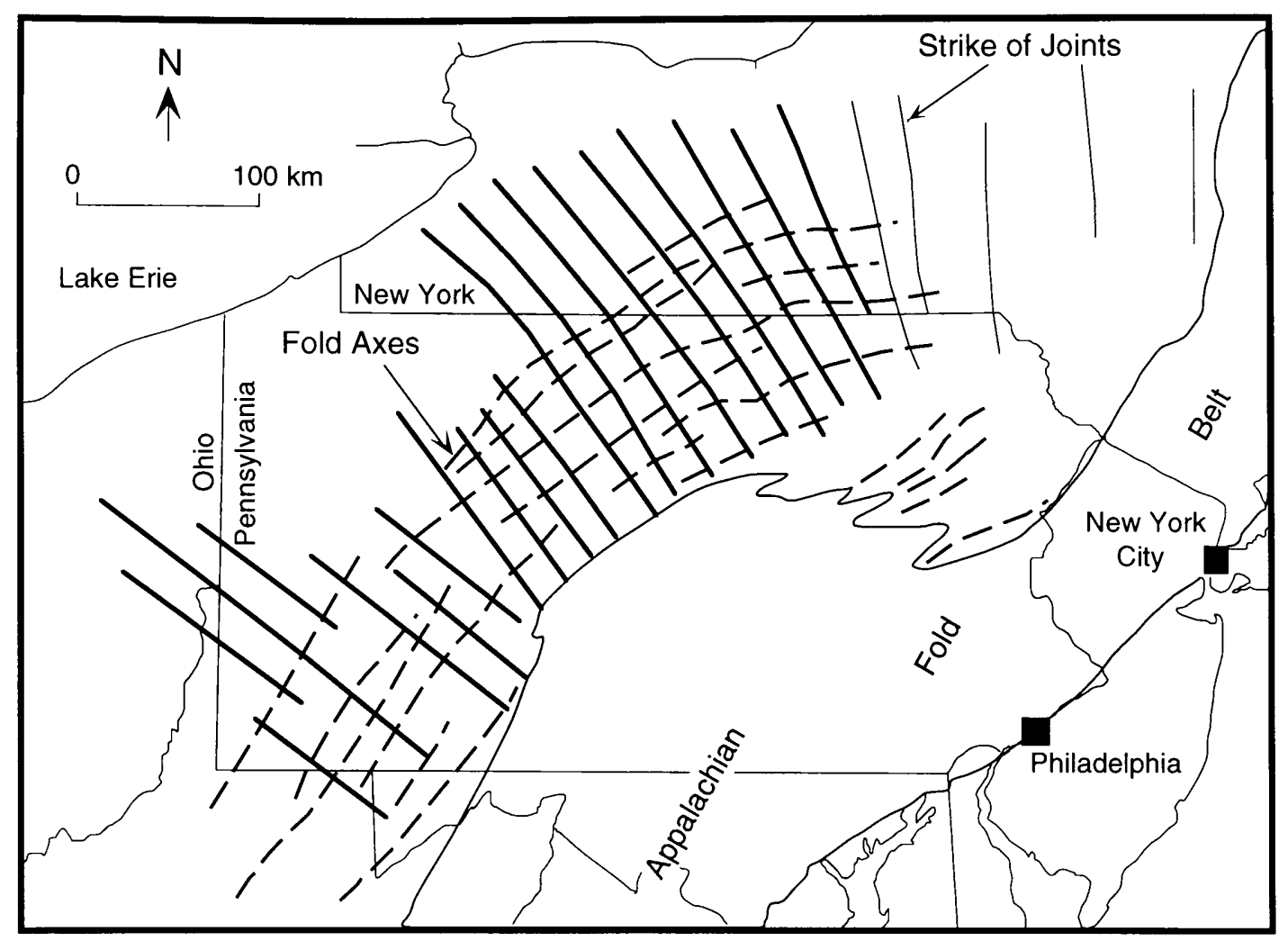

Fig. 1-2. Regional joint pattern on the Appalachian Plateau. Data from Nickelsen and Hough (1967), Rodgers (1970), and Engelder and Geiser (1980). The figure shows strikes of Joint Set Ib as described by Engelder and Geiser (1980). The joint set is perpendicular to the regional fold axes, which formed during the Alleghenian orogeny. The maximum horizontal stress trajectories drawn parallel to joint trends converge southeast toward the hinterland. Redrawn from Suppe (1985). 

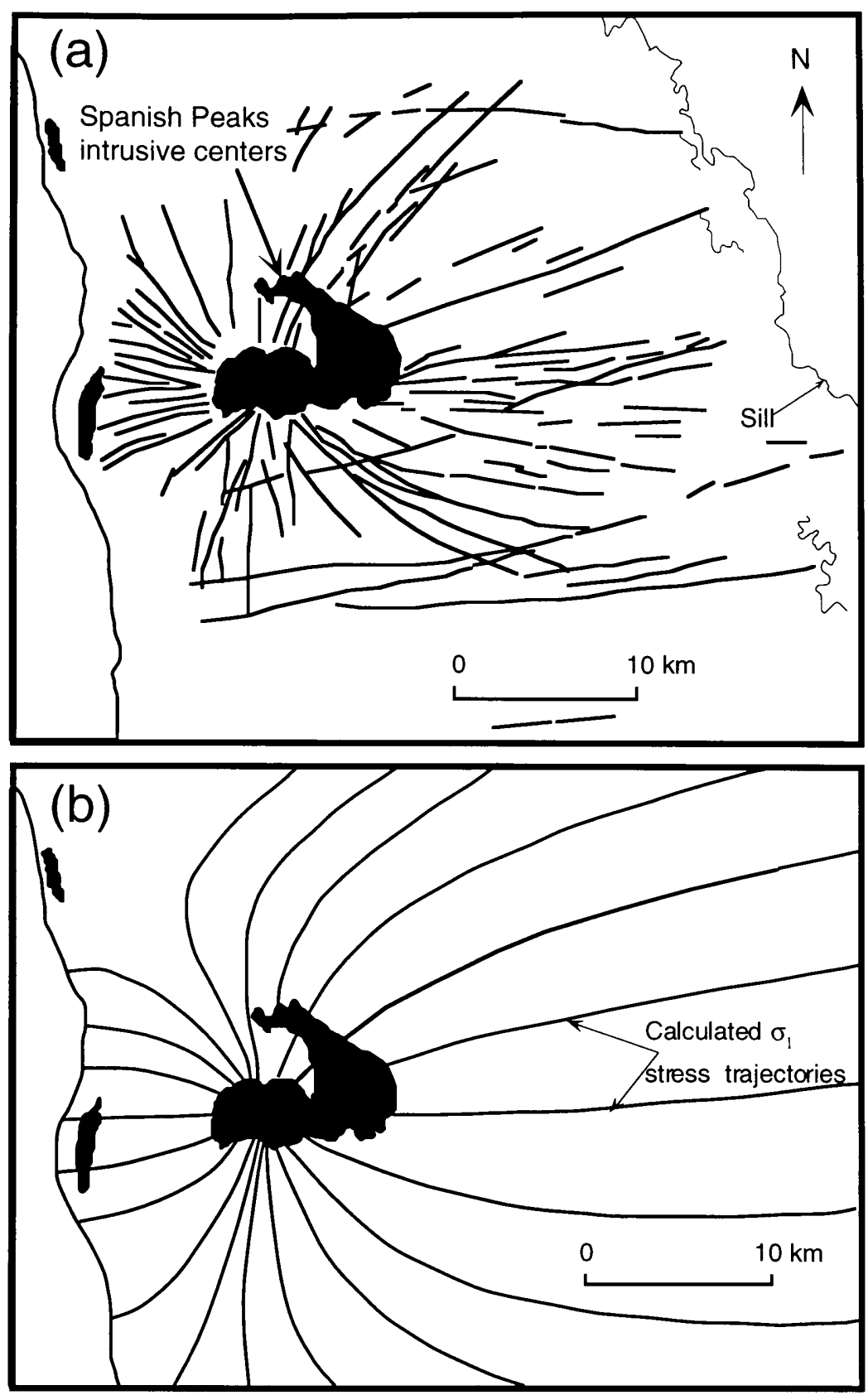

Fig. 1-3. (a) Dike patterns at Spanish Peaks, Colorado (Johnson, 1968).

Theoretical regional stress field at Spanish Peaks (Muller and Pollard, 1977). Note dike trends reflect a combination of local stresses resulting from igneous intrusion in addition to a regional ENE tectonic compressive stress. Redrawn from Suppe (1985). 
development of a fault-bend fold. In yet another example, Olson and Pollard (1989) developed a method to predict relative magnitude of differential stress during joint propagation based on the "hooking geometries" of interacting crack tips.

This thesis focuses on the relationship between state of stress and cross joint geometry. Cross joints are a specific type of fracture that grow in between pairs of pre-existing systematic joints. Their growth is affected by the perturbed stress field adjacent to the pre-existing joints, where local principal stresses often deviate dramatically from the remote stress configuration. As a result, cross joints often exhibit a curving geometry because they are mode-I fractures that propagate normal to local $\sigma_{3}$.

\section{CROSS JOINTS}

According to Hodgson (1961), joints may be classified as systematic or non-systematic, with cross joints representing an important variety of non-systematic joints. Cross joints were originally defined by Hodgson as joints that are subnormal to systematic joints, without cutting across the systematic joints (Fig. 1-4a, Hodgson, 1961). Compared to systematic joints, cross joints often exhibit non-planar surfaces and irregular, curved traces on bedding plane surfaces (Figs. 1-4b \& c, Gross, 1993a). Consequently, they are often less consistent in orientation than systematic joints (Fig. 1-4c). In some locations, however, cross joints also display properties belonging to systematic joints, such as a regular spacing (Gross, 1993a), and planar or curvi-planar surfaces aligned in an en echelon pattern 

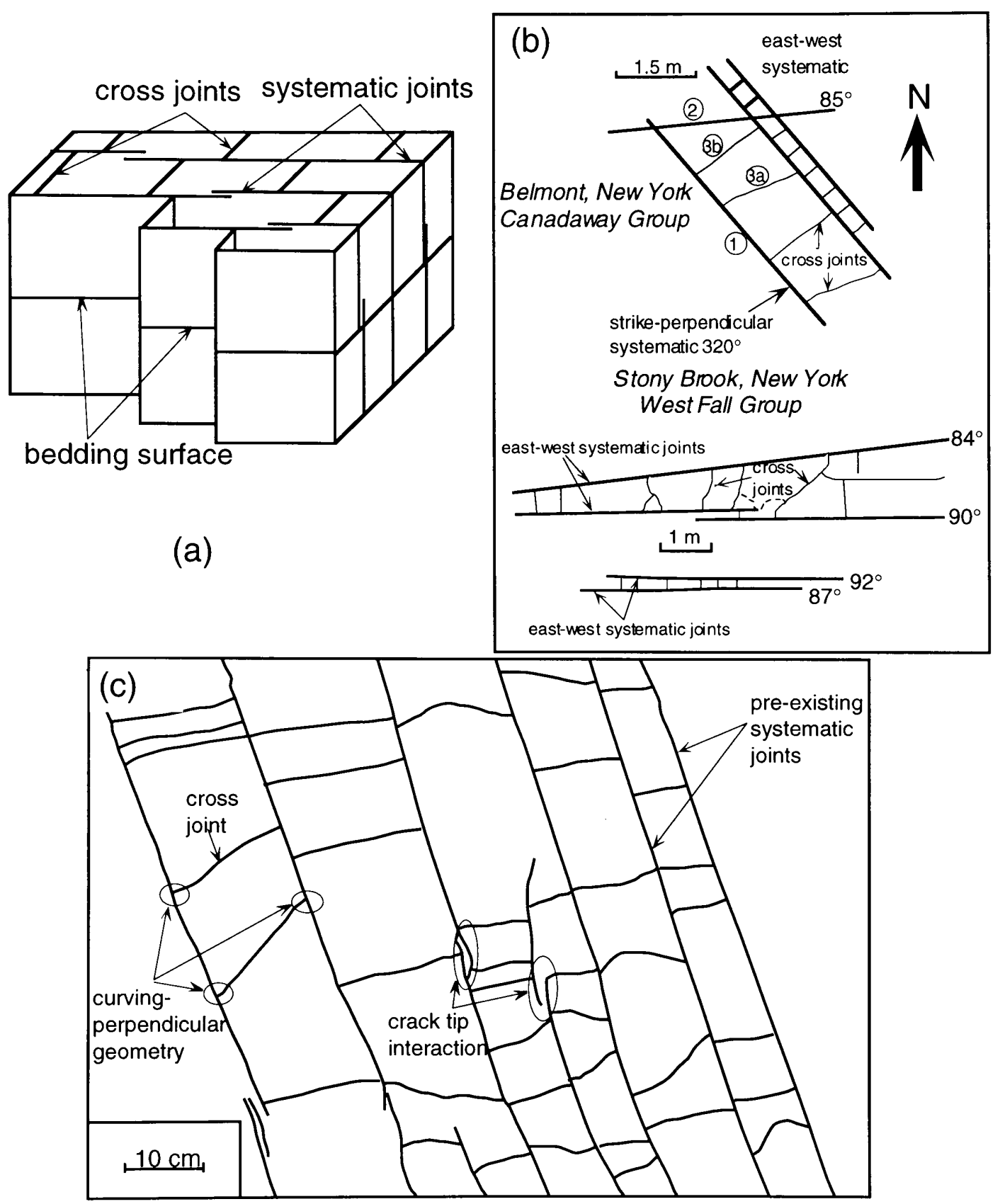

Fig. 1-4. Some cross joint examples from the literature. (a) Schematic block diagram showing cross joints defined by Hodgson (1961) and their relations to systematic pre-existing joints. (b) Curving cross joints observed by Engelder and Gross (1993). (c) Systematic joints and cross joints at Alegria, California (Gross, 1993). 
(Dyer 1988). In most cases, cross joints are initially oriented at high angles to systematic joints (Hodgson 1961; Hancock, 1985; Engelder and Geiser, 1980; Gross, 1993a; Engelder and Gross, 1993), however, low angles (i.e., $<45^{\circ}$ ) between cross joints and systematic joints are also observed in the field (Fig. 1-5).

In order to establish consistent nomenclature, I herein define cross joints as joints that extend across intervals between pre-existing joints, without cutting across the pre-existing joints. Arrays of cross joints typically resemble a "ladder-like" pattern on outcrop, as described by Rawnsley et al (1992). The usage of the term cross joint in this thesis should not be confused with "cross-strike" and "cross fold" coined by Engelder and Geiser (1980) and Srivastava and Engelder (1990) to describe vertical joints trending at high angles to fold axes, nor to the "cross joints" defined by Cloos (1922) to describe lineation-perpendicular joints in granitic plutons. Several important characteristics of cross joints are noteworthy. First, cross joints can be either non-systematic or systematic. Compare, for example, the irregular cross joints in Fig. 1-4c to the regular en echelon patterns observed by Dyer (1988) in Fig. 1-5. Second, cross joints develop in between two pre-existing systematic joints. They may abut against the pre-existing systematic joints, but they do not cut across them. Thus, cross joint lengths are limited by intervals between pre-existing systematic joints. In fact, the distance between adjacent systematic joints often serves as a mechanical layer thickness, which is proportional to cross joint spacing (Gross, 1993a). Third, from their cross-cutting relationship with systematic joints, cross joints clearly formed later in time than the systematic joints. Fourth, the angles between the main segment of the cross 

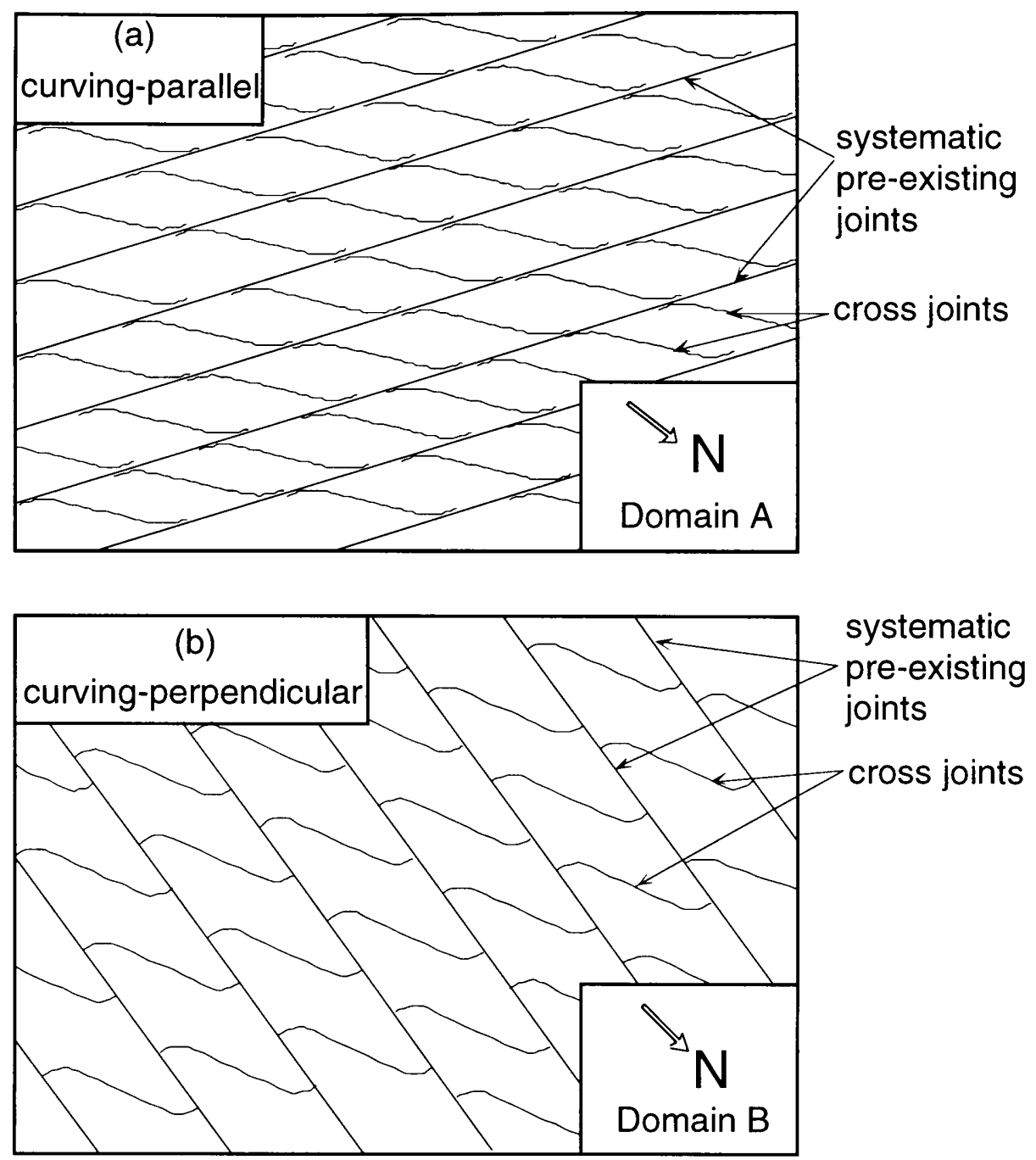

Fig. 1-5. Schematic diagram showing systematic pre-existing joints and cross joints observed by Dyer (1988). (a) Curving-parallel geometry. (b) Curving-perpendicular geometry. Patterns are based on the information provided in Dyer's Fig. 2. Not drawn to scale. 
joints and pre-existing systematic joints can vary from acute to subnormal, depending upon the orientation of the remote stress field in which they formed (see, e.g., Dyer, 1988; Rawnsley et al, 1992; Gross, 1993a).

Because cross joints are oriented differently from, and consistently abut against, pre-existing joints, it is reasonable to assume they neither formed at the same time nor under the same stress conditions. This is because (1) younger joints abut against older joints, and, (2) as mentioned in the introduction, joints are mode-I fractures that always propagate in the direction perpendicular to the least principal stress. Therefore, cross joints in many cases develop in a stress field different from that which prevailed during propagation of the pre-existing systematic set (e.g., Dyer, 1988). Using these principles, Engelder and Gross (1993) proposed that a set of late-formed cross joints can serve as potential indicators of a neotectonic stress field.

Joint abutments (i.e., the intersection points among different joints) have been used to describe a component of the "architectural style" of fractured outcrops. Hancock (1985) noted that shapes often resemble capital letters of the Roman alphabet, as shown schematically in Fig. 1-6. In some cases, joints pass through each other, whereas in other instances one joint terminates against another (e.g., "T", "H", "Y", "A" intersections in Fig. 1-6). In the latter case, cross cutting relationships can be used to determine the relative timing of joint propagation. However, these descriptions do not account for subtle changes in joint geometry near points of intersection.

Field observations demonstrate that in some cases cross joints approach pre-existing joints with a curving-perpendicular geometry 

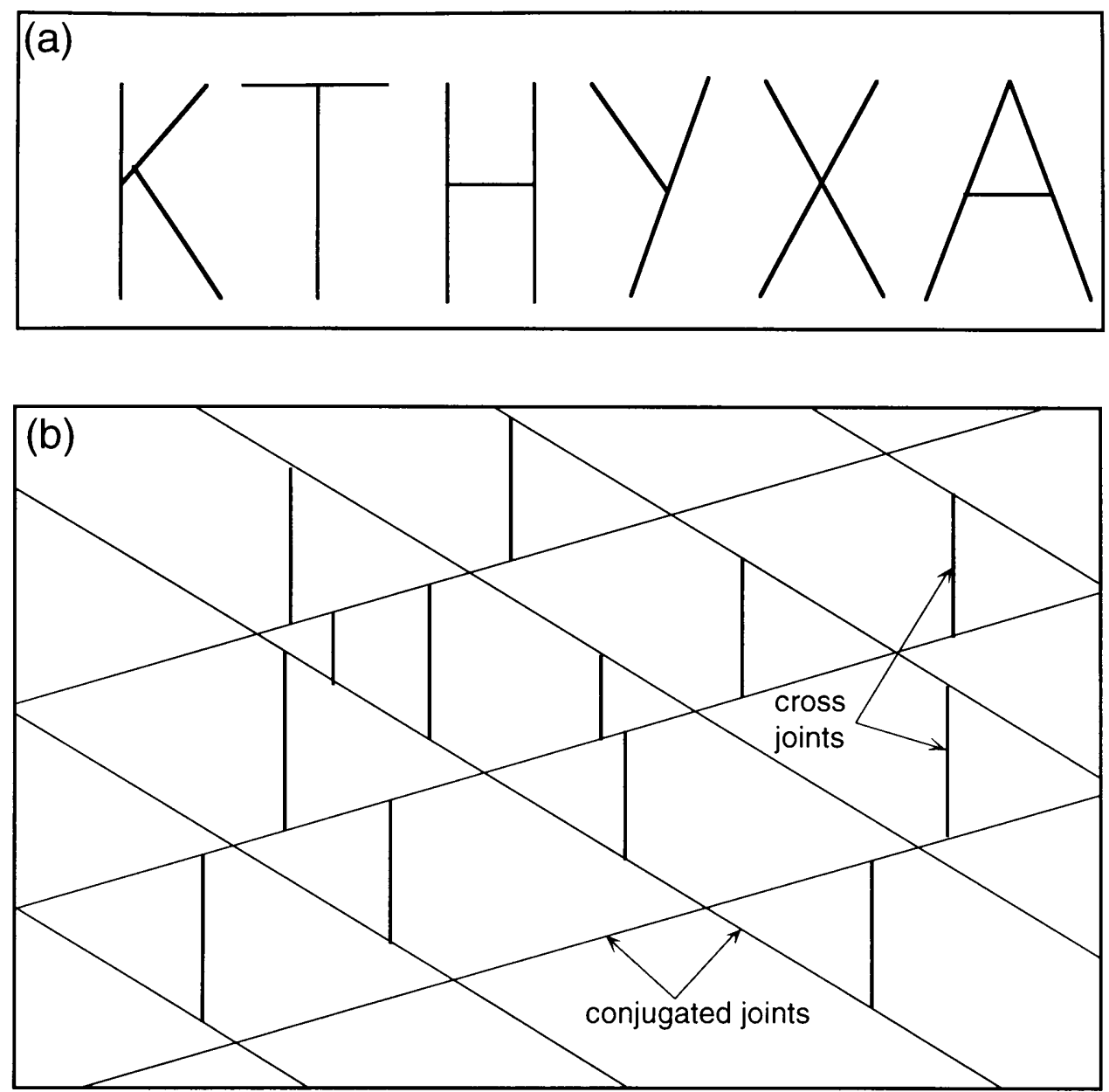

Fig. 1-6. Several of the joint-system architectural styles on bedding planes described by Hancock (1985). (a) Letter shapes of joint abutments.

(b) A-shaped patterns resulting from regular cross joints superimposed on pre-existing conjugated joints. Based on descriptions of Hancock (1985). 
(Figs. 1-4b \& c, Fig. 1-5b,), whereas in other cases a curving-parallel geometry is displayed (Fig. 1-5a). These changes in joint trajectory are thought to arise from local stress perturbations created by the pre-existing joint. The paths taken by cross joints result from a combination of remote and local stresses. Cross joints initiate at some distance away from the preexisting joint, and thus initial trends are strongly controlled by the remote tectonic stress field. However, cross joint paths often deviate from the regional trend in zones adjacent to pre-existing joints due to changes in the local stress field. In other words, initial cross joint trend reflects a change in the orientation of the tectonic stress field subsequent to formation of the systematic joints, whereas the curving geometry indicates changes in local stress orientations encountered during cross joint growth.

\section{GOALS OF THE THESIS}

The first analytical treatment of cross joint geometry was provided by Dyer (1988), who derived relationships for cross joint growth as a function of orientation and relative magnitude of the remote principal stresses. The primary goal of this thesis is to augment Dyer's contributions by solving analytically for the following:

1) The angle of cross joint growth as a function of distance from a preexisting joint, which yields theoretical cross joint trajectories

2) The size of the compressive zone that develops in the vicinity of preexisting joints; this zone tends to inhibit cross joint growth 
3) The influence of internal fluid pressure on the growth and geometry of cross joints; elevated fluid pressures are the dominant mechanism for joint propagation at depth, where all three principal stresses are generally compressive.

Another goal of the thesis is to apply cross joint theory to several field examples. Thus, one can evaluate the usefulness of the theoretical analysis of cross joints in estimating tectonic stress and the effect of mechanical interactions.

Cross joints have received little attention in the geologic literature primarily because they are limited in size and are subsidiary to the more prominent and throughgoing systematic joints. However, cross joints play a critical role in enhancing fluid flow in fractured rock, as they provide connectivity among otherwise isolated joints. Therefore, understanding the formation and resulting geometries of cross joints can lead to a better understanding of the subsurface flow of hydrocarbons, groundwater, and contaminants in rocks that have undergone brittle deformation. In addition, the orientation and relative magnitude of remote stresses derived from the study of cross joints may give important insights into the tectonic development of a region. 


\section{2}

\section{DYER'S (1988) ANALYSIS}

Dyer's field observations revealed that cross joints can either curve in a parallel or a perpendicular fashion as they approach pre-existing joints (Fig. 1-5, Dyer, 1988). He concluded that cross joints form after a rotation of the remote horizontal principal stresses that prevailed during formation of the pre-existing joints. Furthermore, Dyer considered that the initial cross joint trend is parallel to the local maximum horizontal stress, and the curving geometry of cross joints in the vicinity of the pre-existing joints is caused by a perturbation in the local stress field due to the pre-existing joints. Therefore, following the assumption that joints always propagate perpendicular to the least principal stress, he was able to calculate principal stress trajectories in the vicinity of the pre-existing joints and to predict the propagation paths of cross joints (Fig. 2-1).

In Dyer's model the pre-existing systematic joint is represented by a through crack of infinite length and of constant height $(2 c)$ in an infinite 

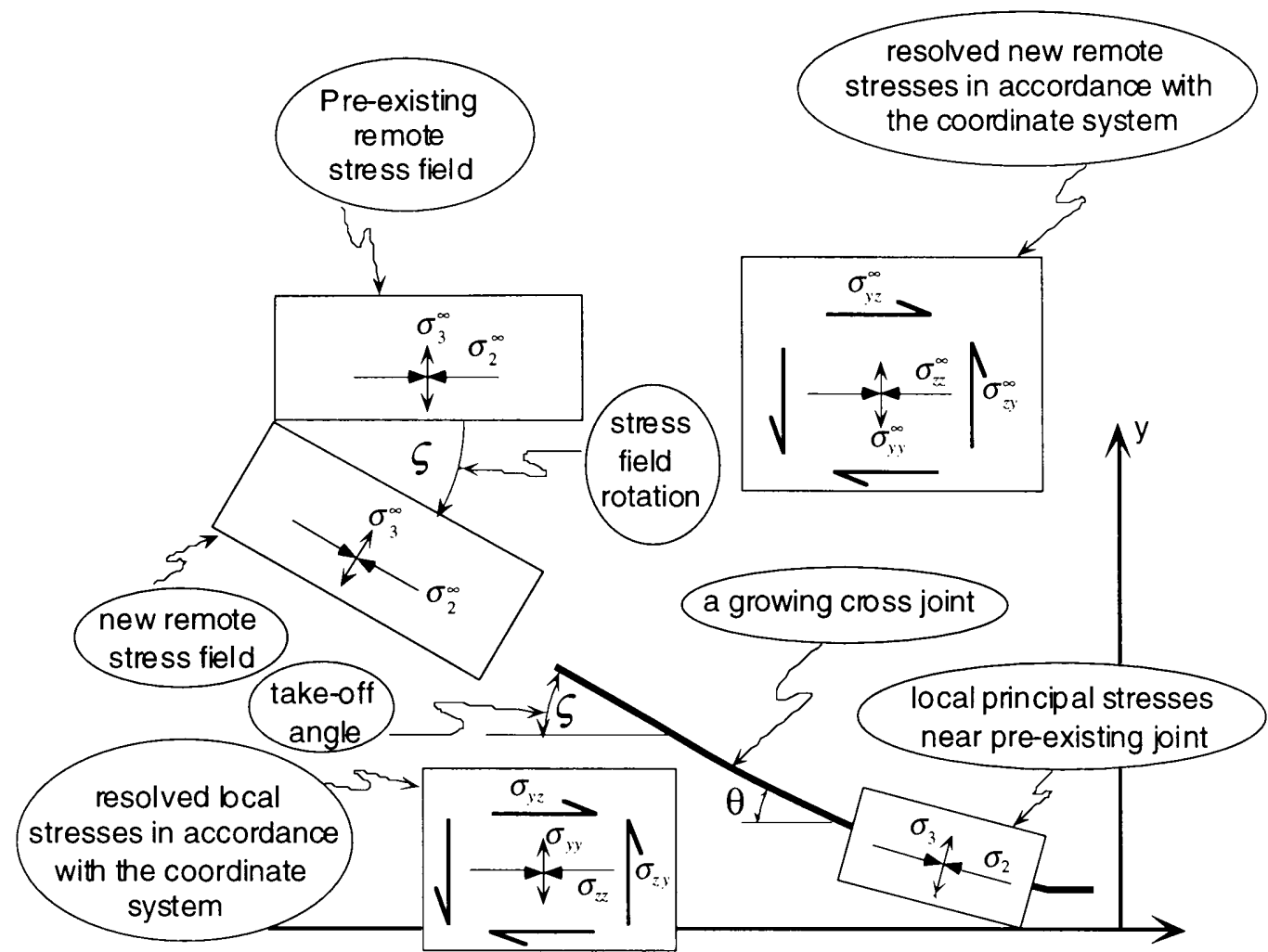

$z$ axis (colinear with the pre-existing fracture)

Fig. 2-1. Dyer's conceptual model. In the pre-existing stress state, $\sigma_{2}^{\infty}$ was parallel to the pre-existing joint, while $\sigma_{3}^{\infty}$ was perpendicular to the pre-existing joint. After formation of the pre-existing joint, the remote stresses rotated through an angle of $\varsigma$. Cross joints formed in accordance with the new stress field. The figure shows that the initial trend of the cross joint is perpendicular to the new remote least principal stress. As the cross joint approaches the pre-existing joint, a curving geometry occurs in response to the local stress field near the pre-existing joint. The figure also shows the resolved remote and local stresses with respect to the coordinate system shown in Fig. 2-2. 


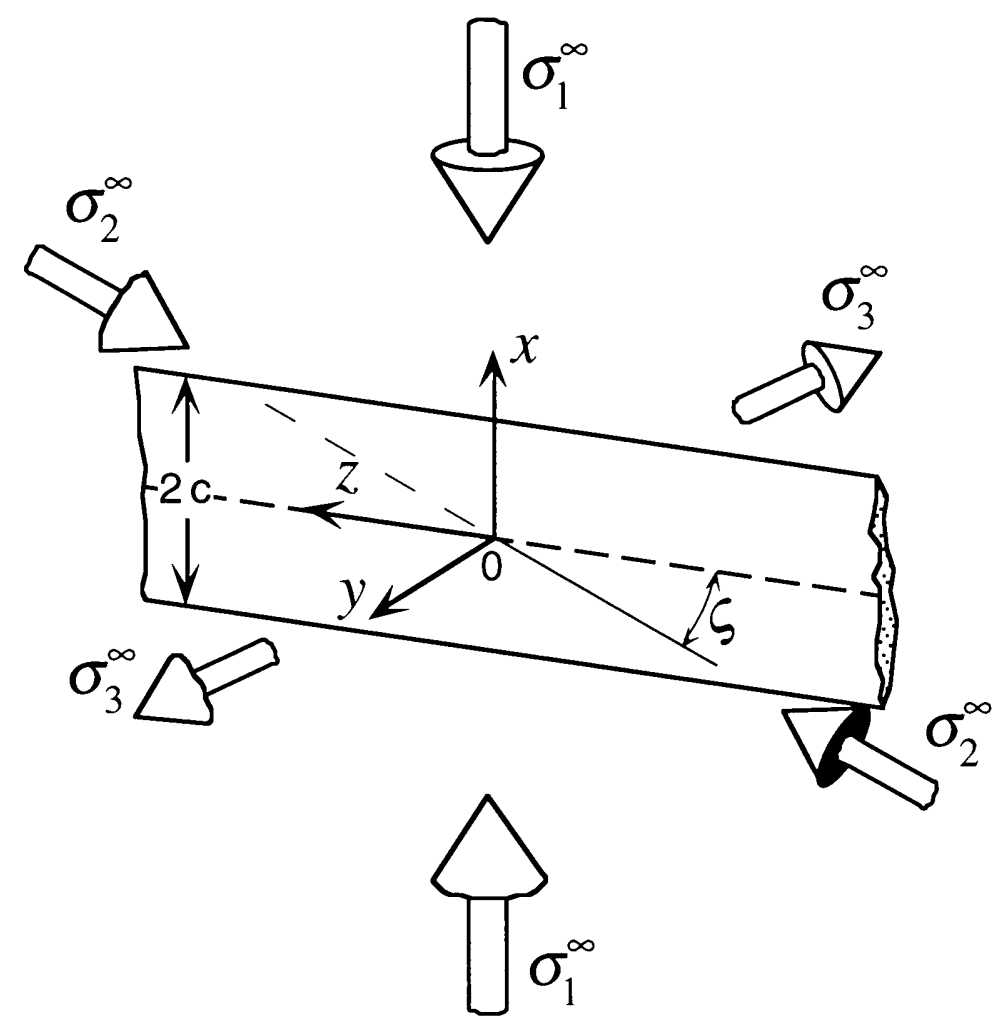

Fig. 2-2. Dyer's stress configuration and coordinate system. The figure shows a joint of infinite length and of height $2 \mathrm{c}$ subjected to a remote stress field in which $\sigma_{1}^{\infty}$ is vertical, and $\sigma_{2}^{\infty}$ and $\sigma_{3}^{\infty}$ are horizontal. After Dyer (1988). 
homogeneous and isotropic elastic medium. The elastic medium containing the pre-existing joint is subjected to remote stresses where the maximum principal stress is vertical, and the intermediate and least principal stresses are horizontal (Fig. 2-2). After the formation of the initial joint set and the subsequent rotation of the tectonic stress about $x$-axis, the horizontal principal stresses become re-aligned, with $\varsigma$ representing the acute angle between the intermediate principal stress and the pre-existing joint. Note that the coordinate system defined by Dyer is left-handed, in which the $x$ axis is vertical and lies in the plane of the pre-existing joint, the $y$-axis is horizontal and perpendicular to the plane of the pre-existing joint, and the $z$-axis is horizontal and lies in the plane of the pre-existing joint (Fig. 2-2).

Dyer made three important assumptions in his theoretical treatment of cross joints. First, a tensile minimum principal stress $\left(\sigma_{3}\right)$ is required for joint growth. In the case of internal fluid pressure, this can be an effective tensile stress. Second, the orientation of the growing cross joint is perpendicular to the local minimum horizontal stress $\left(\sigma_{3}\right)$. Third, there is no interaction between the pre-existing joint and the growing cross joint, which means the stresses around the pre-existing joint are not affected by the presence of the cross joint.

Based on the stress configuration, the coordinate system defined in Fig. 2-2 and using the equations of Mohr circle construction, Dyer first obtained the resolved remote stresses in the directions of the coordinate system (Fig. 2-2). They are

$$
\sigma_{y y}^{\infty}=\left[\left(\sigma_{2}^{\infty}+\sigma_{3}^{\infty}\right)-\left(\sigma_{2}^{\infty}-\sigma_{3}^{\infty}\right) \cos 2\left(90^{\circ}-\varsigma\right)\right] / 2,
$$




$$
\begin{aligned}
& \sigma_{z z}^{\infty}=\left[\left(\sigma_{2}^{\infty}+\sigma_{3}^{\infty}\right)+\left(\sigma_{2}^{\infty}-\sigma_{3}^{\infty}\right) \cos 2\left(90^{\circ}-\varsigma\right)\right] / 2, \\
& \sigma_{y z}^{\infty}=\left[\left(\sigma_{2}^{\infty}-\sigma_{3}^{\infty}\right) \sin 2\left(90^{\circ}-\varsigma\right)\right] / 2,
\end{aligned}
$$

(Dyer's equation 1, 2, 3). The "take-off" angle, $\varsigma$, is the angle between the maximum horizontal stress and the pre-existing joint (i.e., $z$-axis, Fig. 2-2), $\sigma_{2}^{\infty}$ and $\sigma_{3}^{\infty}$ are the maximum and minimum remote horizontal stresses respectively, and $\sigma_{y y}^{\infty}, \sigma_{z z}^{\infty}$ and $\sigma_{y z}^{\infty}$ represent the remote normal stresses and the shear stress corresponding to the coordinate system in Fig. 2-2.

Dyer assumed that the pre-existing joint is open when $\sigma_{y y}^{\infty}$ is tensile $\left(\sigma_{y y}^{\infty}<0\right)$ and closed when $\sigma_{y y}^{\infty}$ is compressive $\left(\sigma_{y y}^{\infty}>0\right)$. This division results in four specific stress states relevant to cross joint growth (Fig. 2-3). There are two subclasses in the open case according to the value of $\sigma_{z 2}^{\infty}$. When $\sigma_{z z}^{\infty}>0$, curving-parallel geometry occurs, whereas when $\sigma_{z z}^{\infty}<0$, curving-perpendicular geometry occurs.

In the closed case, the opposing rock walls are in contact, and two subclasses can also be distinguished based on the coefficient of friction along the pre-existing joint. One is the frictional sliding case (i.e., a shear stress exists along the pre-existing joint), where the frictional coefficient of the pre-existing joint is not zero. The other is the frictionless case, where there is no shear stress on the joint surface, and hence the coefficient of friction is zero.

Using the above considerations, Dyer then analytically solved for the local stresses with respect to the coordinate system (Fig. 2-2) in the $x=0$ plane, which for the open case are: 


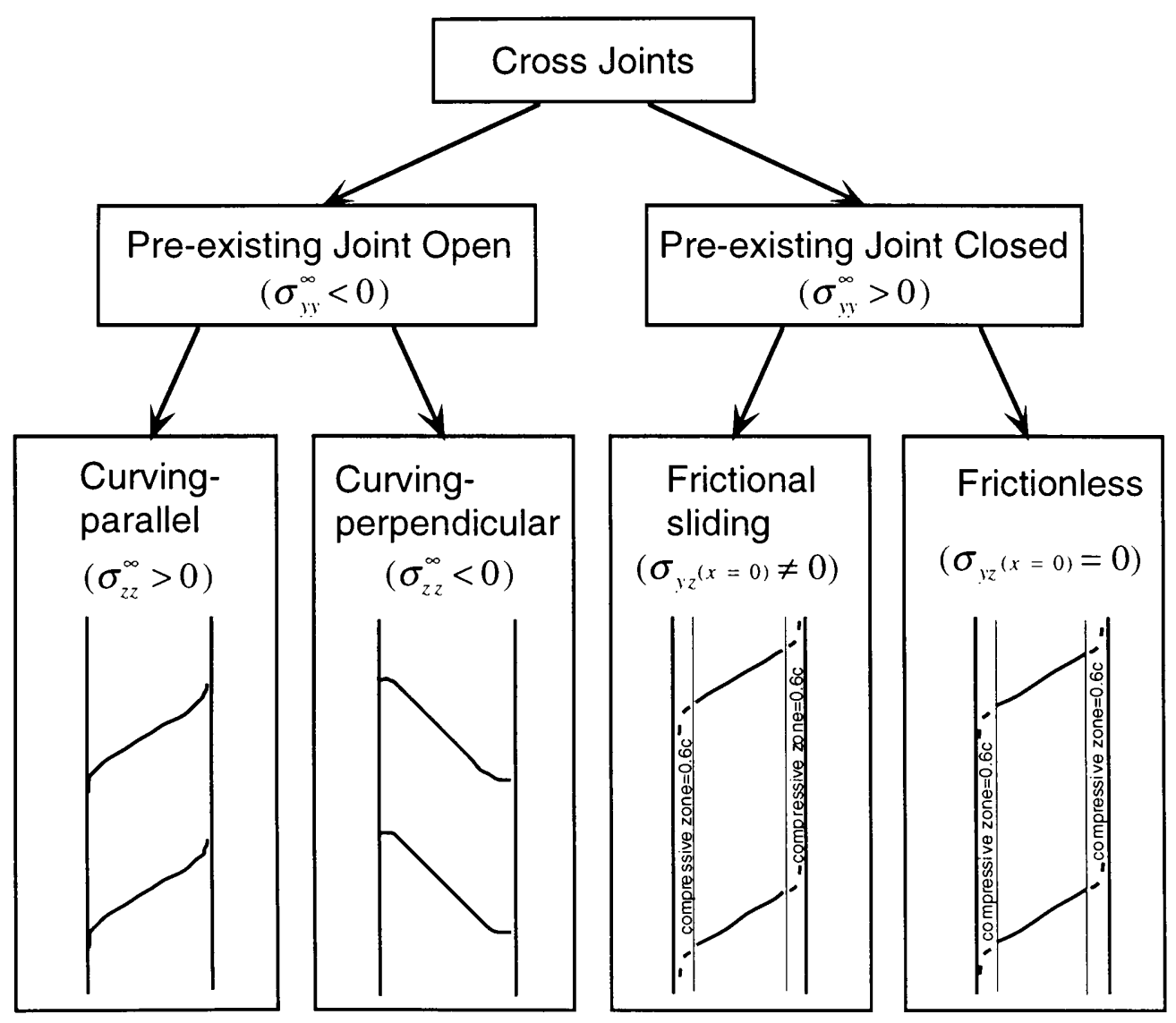

Fig. 2-3. The four cross joint subclasses of Dyer (1988). Note that cross joints are inhibited from growing in compressive zones. 


$$
\begin{aligned}
& \sigma_{y y}(x=0)=\sigma_{y y}^{\infty}\left[\left|y^{3}\right| /\left(y^{2}+c^{2}\right)^{3 / 2}\right], \\
& \sigma_{x x}(x=0)=\sigma_{y y}^{\infty}\left[|y|\left(y^{2}+2 c^{2}\right)^{3 / 2} /\left(y^{2}+c^{2}\right)^{3 / 2}-1\right], \\
& \sigma_{y z}(x=0)=\sigma_{y z}^{\infty}\left[|y| /\left(y^{2}+c^{2}\right)^{1 / 2}\right], \\
& \sigma_{z z}(x=0)=\bar{\sigma}_{z z}^{\infty}+v\left(\sigma_{x x}+\sigma_{y y}\right),
\end{aligned}
$$

(Dyer's equation 9, 10, 11, 12), and for the closed case are:

$$
\begin{aligned}
& \sigma_{y y}=\sigma_{y y}^{\infty}, \\
& \sigma_{x x}=\sigma_{x x}^{\infty}, \\
& \sigma_{y z}=\sigma_{y z}^{\infty}\left[s+(1+s) y /\left(y^{2}+c^{2}\right)^{1 / 2}\right], \\
& \sigma_{z z}(x=0)=\bar{\sigma}_{z z}^{\infty}+v\left(\sigma_{x x}+\sigma_{y y}\right),
\end{aligned}
$$

which are the equation 21 and equation group 22 in Dyer's (1988) paper. The terms $\sigma_{x x}, \sigma_{y y}$ and $\sigma_{z z}$ are local normal stresses, $\sigma_{y z}$ is local shear stress corresponding to the coordinate system defined in Fig. $2-2, \quad \bar{\sigma}_{z z}^{\infty}$ is the remote stress in the direction of $z$ axis (i.e., parallel to the pre-existing joint), $c$ is the half height of the pre-existing joint, and $v$ is Poisson ratio of the elastic medium. Friction is accounted through the shear stress ratio $(s)$, defined as $s=\sigma_{y z}(x=0) / \sigma_{y z}^{\infty}$, where $\sigma_{y z}(x=0)$ is the shear stress along the pre- 
existing joint. Because $\sigma_{y z}(x=0)=C_{0} \sigma_{y y}^{\infty}$, we have $s=C_{0} \sigma_{y y}^{\infty} / \sigma_{y z}^{\infty}$, where $C_{0}$ is the coefficient of friction belonging to the pre-existing joint surface.

After analytically deriving the magnitudes of local stresses as functions of distance from the pre-existing joint and remote stresses, Dyer then used the standard Mohr circle construction to determine the local principal stresses for the special case of $\varsigma=30^{\circ}$, which is the angle between the maximum horizontal stress and the pre-existing joints.

In the open case (i.e., $\sigma_{v v}<0$ ) with $\varsigma=30^{\circ}$, he found that (1) curvingparallel geometry occurs when the remote principal stress ratio is between -3 and $-1 / 3$ (Fig. 2-4a); (2) curving-perpendicular geometry occurs when the remote principal stress ratio is between $-1 / 3$ and 1 (Fig. 2-4b); and (3) there is no compressive zone in this case (Fig. 2-4a \& b). In the closed case, systematic changes in the directions of the local principal stresses also occur (Fig. 2-5a \& b), however a compressive zone exists in both the frictional sliding and frictionless subclasses. The width of this compressive zone is approximately $0.6 c$ (Fig. $2-5 a \&$ b).

From the above description, we can see that Dyer's work provides an excellent first order approximation of the relationships between cross joint geometry and remote principal stress ratios. These simple results can be easily applied to outcrops in an attempt to map the tectonic stress field. The initial cross joint trend gives the orientation of the maximum horizontal stress, and the curving geometry provides the relative magnitude of the ratio between the maximum and minimum horizontal stresses.

Several workers have applied Dyer's study of cross joints in Arches National Park, Utah, to cross joints found elsewhere. Gross (1993a) studied cross joints in the Monterey Formation of California, and concluded that the 


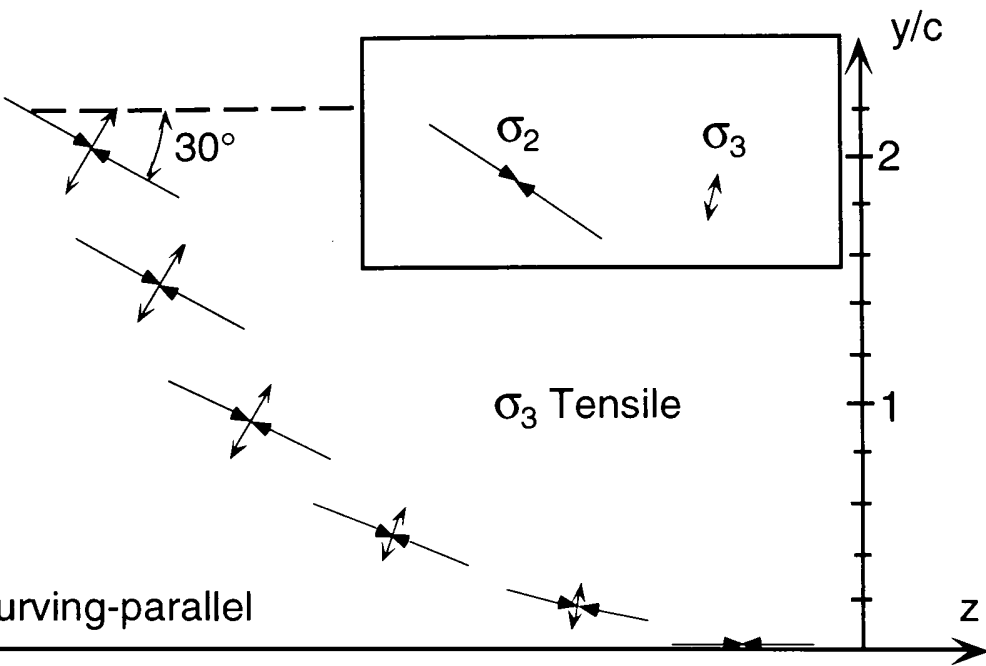

(a) curving-parallel

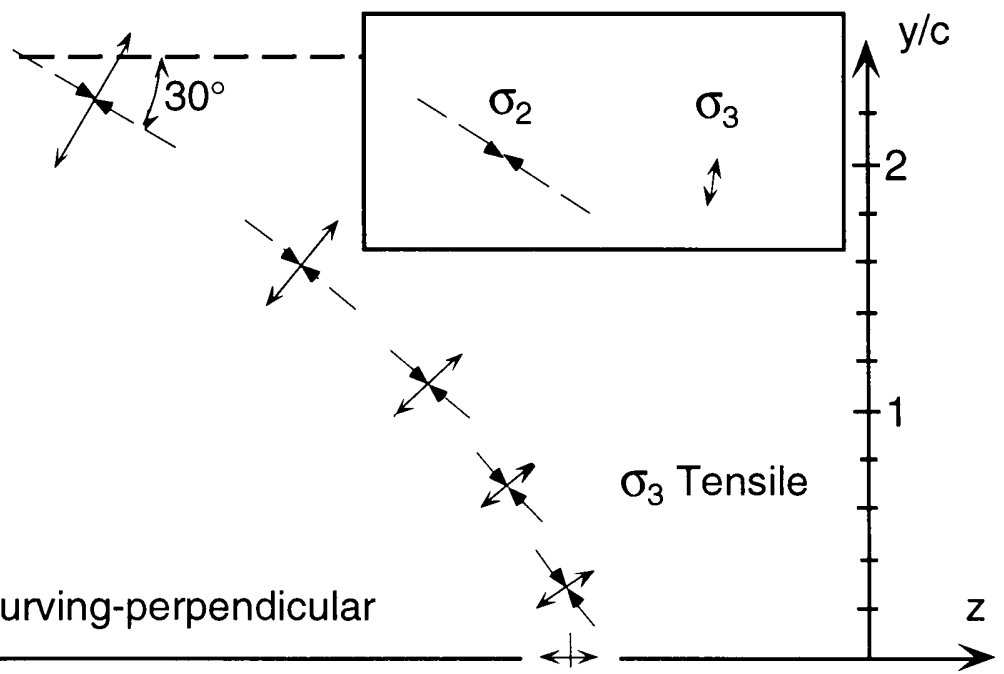

Fig. 2-4. Dyer's curving-parallel and curving-perpendicular cases for an open pre-existing joint. (a) Curving-parallel case. (b) Curvingperpendicular case. The figures show the orientations of local principal stresses as a function of normalized distance from the preexisting joint $(y / c)$. The remote principal stress ratio is -2 . The angle between the pre-existing joint (collinear with $z$ axis) and the remote maximum horizontal stress is $30^{\circ}$. Note $\sigma_{3}$ remains tensile everywhere in both cases. After Dyer (1988). 


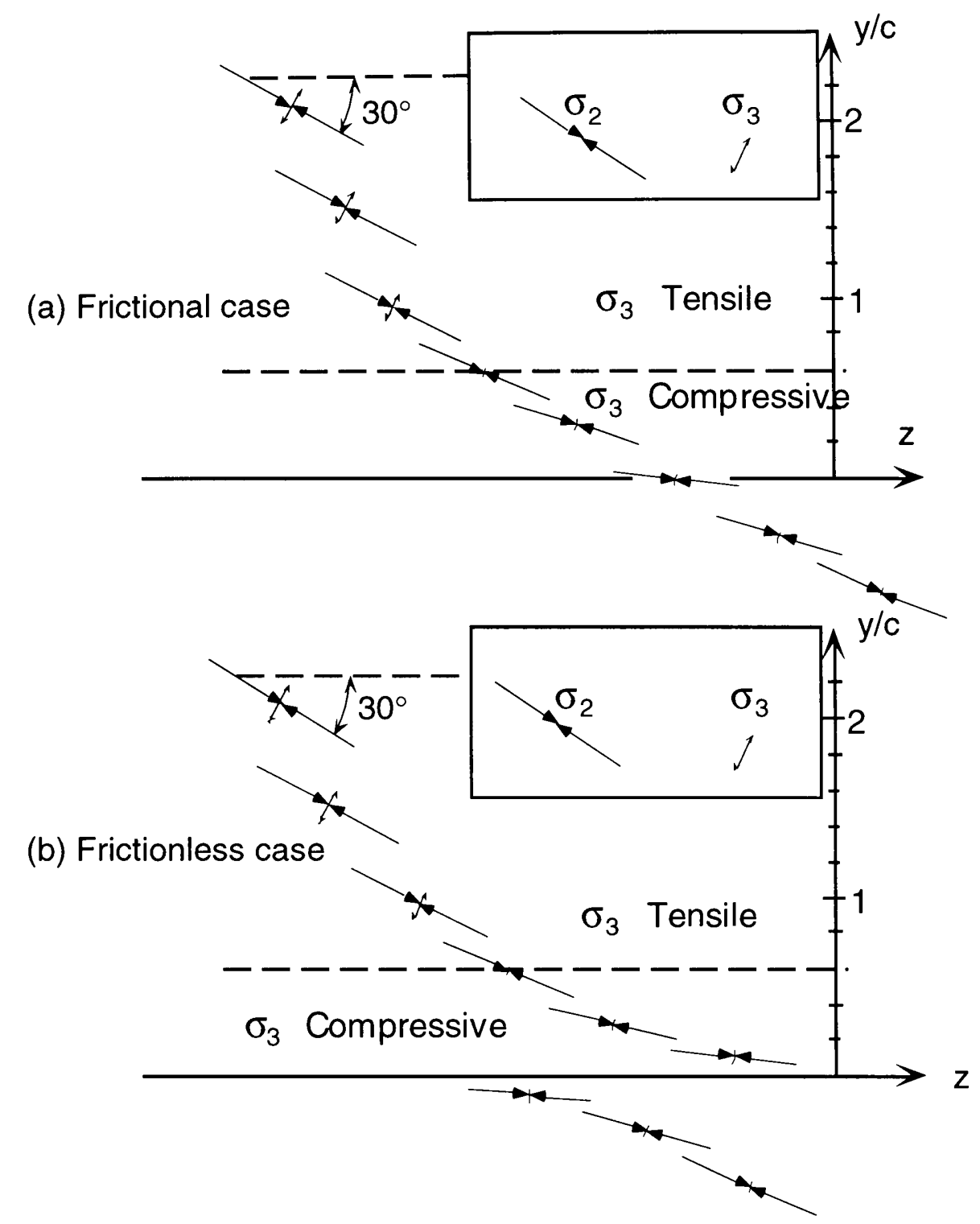

Fig. 2-5. Dyer's frictional and frictionless cases for a closed pre-existing joint. (a) Frictional case. (b) Frictionless case. The remote principal stress ratio is -5 . Note the change in principal stress orientations. The angle between the pre-existing joint (collinear with $z$-axis) and the remote maximum stress is $30^{\circ}$. In the frictional case, a Byerlee-type friction law with a coefficient of 0.6 is used. Note $\sigma_{3}$ changes sign at $y / c \approx 0.6$ in both cases. After Dyer (1988). 
ratio of maximum stress to minimum stress in the bedding plane was between $-1 / 3$ and 1 during the formation of the cross joints. In another example, Engelder and Gross (1993) studied the orientations of the neotectonic stress field and the differential stress based on cross joint traces observed on bedding-plane surfaces of Devonian Catskill clastic rocks of the Appalachian Plateau of western New York state. These studies demonstrate the applicability of Dyer's approach to studying both the orientations and relative magnitudes of stress fields.

Dyer's study, however, focused primarily on a special case in which the angle between the initial cross joint trend and the pre-existing joint is $30^{\circ}$. Furthermore, relationships between cross joint geometry and fluid pressure were not fully considered in his study. Thus, there are several limitations to the general application of Dyer's analysis. For example, what is the range of the remote principal stress ratio when the takeoff angle is a value other than $30^{\circ}$ ? How does cross joint geometry vary with depth where the principal stresses are all compressive? What is the meaning of a cross joint geometry which is neither curving-parallel nor curvingperpendicular? Is the width of the compressive zone in the closed case always approximately $0.6 c$ ? What controls the width of this compressive zone? In the following chapters, I will attempt to answer these questions. 


\section{2 \\ Chap?

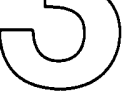

\section{THEORETICAL DERIVATION}

The goal of this chapter is to study the relationships between cross joint geometry and remote principal stresses as well as pore pressure. To achieve this goal, I first clarify the theoretical model and assumptions used in the derivations. Then, based on the defined theoretical model and coordinate system, I discuss the general conditions for open and closed preexisting joints. After that, I solve for cross joint geometry as a function of remote principal stress for the open and closed pre-existing joint cases, respectively. Based on the assumption that joints cannot propagate into a compressive area (i.e., where all principal stresses are compressive), I discuss constrains on cross joint propagation in the fourth step. Finally, I examine the role of pore pressure in controlling cross joint geometry by defining the concept of the effective remote stress ratio. The term "remote stresses" refers to stress configurations located at a point sufficiently far away from any pre-existing joint, such that it is mechanically unaffected by 
a pre-existing joint. Remote stresses do not necessarily coincide with the tectonic stresses. Rather, "remote stresses" is used in this study to mean the same as "far field stresses" in the mechanics literature, which was defined based on the Saint-Venant Principal (Timoshenko and Goodier, 1970).

\section{COORDINATE SYSTEM AND ASSUMPTIONS IN THIS STUDY}

Before proceeding with analytical solutions for cross joint geometries, I outline the boundary conditions and assumptions in the model to be employed, which differ slightly from those of Dyer's (1988) analysis. Following Dyer's example, my model supposes a through crack of infinite length and of constant height (2c) formed in an infinite homogeneous and isotropic elastic medium. This crack represents the systematic joint that existed prior to cross joint propagation (Fig. 3-1), and in layered rocks the height (2c) often corresponds to bed thickness (e.g., Price, 1966; Narr and Suppe, 1991). Whereas Dyer used a left-handed coordinate system (Fig. 22), I designate a right-handed coordinate system where the $x$-axis is vertical and lies in the plane of the pre-existing joint, the $y$-axis is horizontal and perpendicular to the plane of the pre-existing joint, and the $z$-axis is horizontal and lies in the plane of the pre-existing joint (Fig. 3-1).

In order to avoid unnecessary complications with theoretical derivations, the model considers the simple geologic case of vertical fractures developing in horizontal strata. Thus, the pre-existing joint developed in a stress field where the maximum principal stress was vertical, the medium principal stress was horizontal and parallel to the pre-existing 

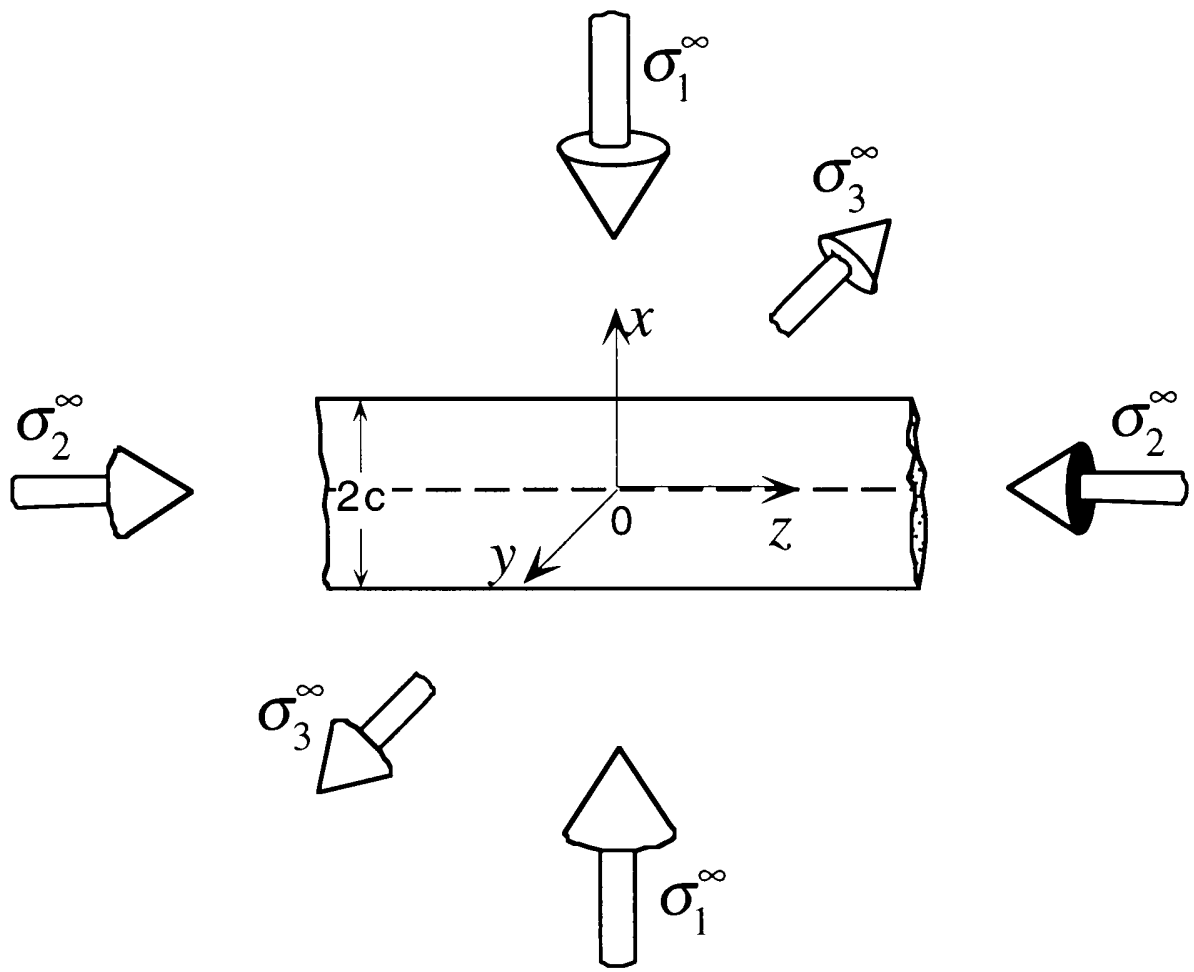

Fig. 3-1. Coordinate system and stress state that prevailed during formation of the pre-existing joint adopted for use in this study. Note that the coordinate system is right-handed in contrast to Dyer's left-handed coordinate system in Fig. 2-2. 
joint, and the least principal stress was horizontal and perpendicular to the pre-existing joint (Fig. 3-1). Due to temporal changes in the remote stress field, the principal stress directions changed subsequent to formation of the pre-existing joint. Suppose there was a clockwise rotation in the horizontal principal stress directions as shown in Fig. 3-2, then new joints would form in accordance with the new remote stress field. Here I designate the maximum, medium and least principal stresses of the new remote stress field as $\sigma_{1}^{\infty}, \sigma_{2}^{\infty}$ and $\sigma_{3}^{\infty}$, respectively. The initial trend of the new joints, which are the cross joints, lies in the plane of $\sigma_{1}^{\infty}$ and $\sigma_{2}^{\infty}$ as shown in Fig. 3-2. The angle between the pre-existing joint and the initial trend of the cross joint is called the takeoff angle, $\varsigma$.

In all the following derivations, I will use three assumptions, which are: (1) a cross joint path is perpendicular to the local least horizontal stress in terms of the theoretical model (Fig. 3-2), which is based on the definition of joints (Pollard and Segall, 1987), (2) there is no interaction between a growing cross joint and the pre-existing joint, and (3) there is no interaction between two adjacent cross joints. Assumptions (2) and (3) mean that the stress field in the vicinity of the pre-existing joint is not affected by introducing any cross joint. Therefore, cross joint paths can be constructed just based on stress trajectories. 


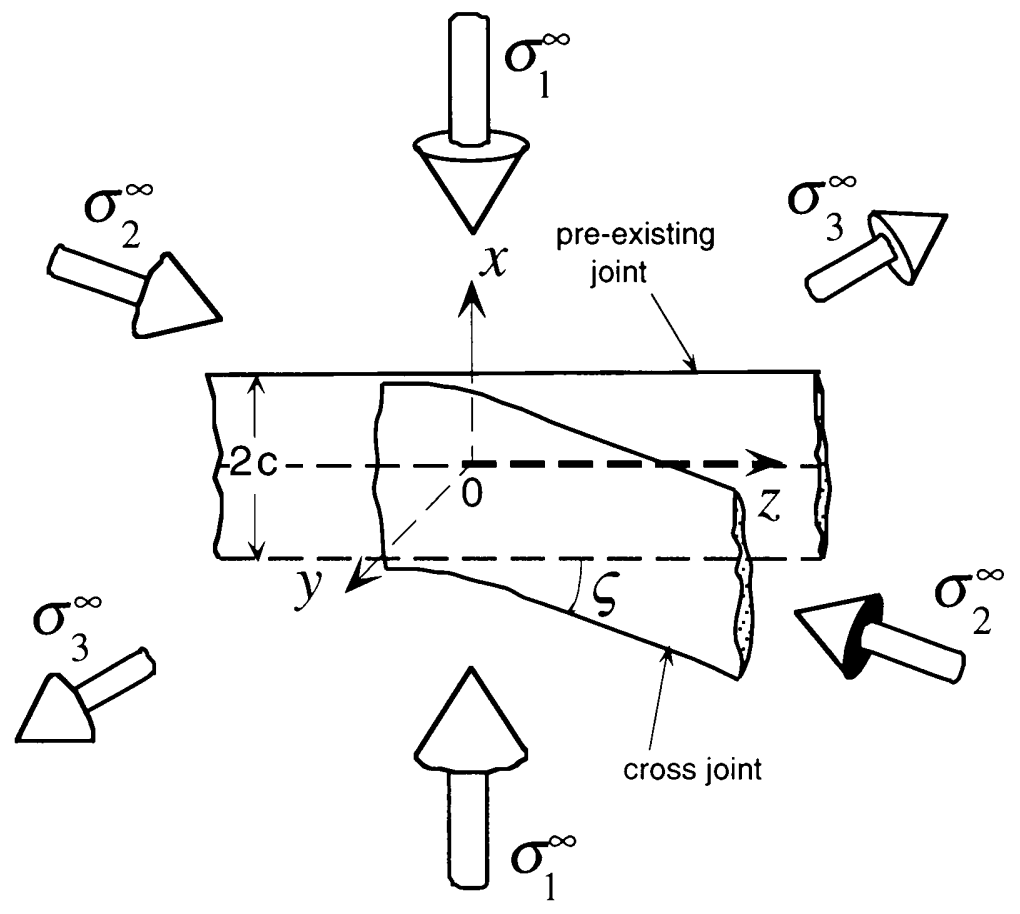

Fig. 3-2. Formation of a cross joint after rotation of the horizontal remote principal stresses. The figure shows the coordinate system, the rotated remote horizontal stresses, the pre-existing joint and a cross joint with a curving-parallel geometry. 


\section{CONDITIONS FOR OPEN AND CLOSED \\ PRE-EXISTING JOINTS}

In Dyer's special case where the take-off angle $(\varsigma)$ equals $30^{\circ}$, curving-parallel geometries occur when the ratio of maximum to minimum horizontal stress (i.e., $\sigma_{2}^{\infty} / \sigma_{3}^{\infty}$ ) ranges from -3 to $-1 / 3$ (Figs. $2-4,2-5$ ). On the other hand, curving perpendicular geometries occur when the ratio is $-1 / 3$ to 1 . In the following analysis I consider the general case in which the cross joint initiates from an arbitrary take-off angle.

Based on the coordinate system, the stress configuration defined in Fig. 3-2 and using the equations of Mohr circle construction (Jaeger and Cook, 1979), one can obtain the resolved remote stresses in the directions of the coordinate system. They are

$$
\begin{aligned}
& \sigma_{y y}^{\infty}=\left[\left(\sigma_{2}^{\infty}+\sigma_{3}^{\infty}\right)-\left(\sigma_{2}^{\infty}-\sigma_{3}^{\infty}\right) \cos 2 \varsigma\right] / 2, \\
& \sigma_{z z}^{\infty}=\left[\left(\sigma_{2}^{\infty}+\sigma_{3}^{\infty}\right)+\left(\sigma_{2}^{\infty}-\sigma_{3}^{\infty}\right) \cos 2 \varsigma\right] / 2, \\
& \sigma_{y z}^{\infty}=\left[\left(\sigma_{2}^{\infty}-\sigma_{3}^{\infty}\right) \sin 2 \varsigma\right] / 2 .
\end{aligned}
$$

Here $\sigma_{y y}^{\infty}, \sigma_{z z}^{\infty}$ and $\sigma_{y z}^{\infty}$ represent the remote normal stresses and the shear stress corresponding to our coordinate system in Fig. 3-2. These solutions are slightly different from Dyer's solutions (shown in equations 2-1, 2-2 and 2-3). In fact, there is a minor mistake in Dyer's equations, and $\sigma_{z z}^{\infty}$ of his solution should be replaced by $\sigma_{y y}^{\infty}$. 
According to Dyer (1988), when $\sigma_{y y}^{\infty}$ is tensile (i.e., $\sigma_{y y}^{\infty}<0$ ), the preexisting joint is open. In contrast, the pre-existing joint is closed when $\sigma_{y}^{\infty}$ is compressive (i.e., $\sigma_{y v}^{\infty}>0$ ). Using equation (3-1), and considering the conditions in forming the cross joints $\left(\sigma_{3}^{\infty}<0\right)$, as well as the convention $\sigma_{2}^{\infty} \geq \sigma_{3}^{\infty}$, the condition for an open pre-existing joint is

$$
-(1+\cos 2 \varsigma) /(1-\cos 2 \varsigma)<\sigma_{2}^{\infty} / \sigma_{3}^{\infty} \leq 1,
$$

and the condition for a closed pre-existing joint is

$$
\sigma_{2}^{\infty} / \sigma_{3}^{\infty}<-(1+\cos 2 \varsigma) /(1-\cos 2 \varsigma) .
$$

Note that $\cos 2 \varsigma \geq 0$ when $0^{\circ} \leq \varsigma \leq 45^{\circ}$, and $\cos 2 \varsigma \leq 0$, when $45^{\circ} \leq \varsigma \leq 90^{\circ}$. For convenience in the following discussion, the absolute value of $\cos 2 \varsigma$ is used to define the conditions for open and closed pre-existing joints. The conditions for an open and a closed pre-existing joint are shown in Table 31. From Table 3-1, we can see that an open or closed status for the preexisting joint not only depends upon the remote principal stress ratio, but also upon the takeoff angle $\varsigma$. For example, when the remote principal stress ratio is -2 , the pre-existing joint is open when $\varsigma=30^{\circ}$. However, for the same stress ratio, the pre-existing joint is closed when $\varsigma=60^{\circ}$. 
Table 3-1. Conditions for open and closed pre-existing joints. In this table, $\varsigma$ represents the take off angle, $\sigma_{2}^{\infty} / \sigma_{3}^{\infty}$ is the remote principal stress ratio. The numbers in the parentheses are just for the convenience in the following discussions. $S=-(1+|\cos 2 \varsigma|) /(1-|\cos 2 \varsigma|)$, $T=-(1-|\cos 2 \varsigma|) /(1+|\cos 2 \varsigma|)$.

\begin{tabular}{||c|c|c|c||}
\hline & $\frac{\sigma_{2}^{\infty}}{\sigma_{3}^{\infty}}<\mathrm{S}$ & $\mathrm{S}<\frac{\sigma_{2}^{\infty}}{\sigma_{3}^{\infty}}<\mathrm{T}$ & $\mathrm{T}<\frac{\sigma_{3}^{*}}{\sigma_{3}^{\infty}} \leq 1$ \\
\hline $0^{\circ}<\varsigma<45^{\circ}$ & Closed Case \#1 & Open Case \#1 & Open Case \#2 \\
\hline $45^{\circ}<\varsigma<90^{\circ}$ & Closed Case \#2 & Closed Case \#3 & Open Case \#3 \\
\hline
\end{tabular}




\section{CROSS JOINT GEOMETRY AS A FUNCTION OF REMOTE PRINCIPAL STRESS RATIO}

The goal of this section is to derive expressions that outline cross joint propagation paths as a function of relative stress magnitudes and orientations. This is accomplished by discussing the two different cases, i.e., open and closed pre-existing joint cases, respectively.

\section{OPEN PRE-EXISTING JOINT CASE}

In discussing the open pre-existing joint case, a three-step procedure is used. The first step is to define the general conditions for curvingparallel and curving-perpendicular geometries as a function of remote stress ratio and take-off angle. The second step solves for the stress components adjacent to the pre-existing joint, and then determines the orientation of local maximum horizontal stress as a function of distance from the preexisting joint. Based on the assumption that cross joint traces are aligned parallel to maximum horizontal principal stress, the third step plots expected cross joint trajectories as a function of stress ratios for selected take-off angles.

\section{(1). Conditions for curving-parallel and curving-perpendicular geometries}

In his analysis, Dyer (1988) concluded that both curving-parallel and curving-perpendicular geometries can occur when $\sigma_{v i}^{\infty}<0$. The difference 
in geometries of these cross joints are controlled by $\sigma_{z z}^{\infty}$. When $\sigma_{z z}^{\infty}>0$, a curving-parallel geometry occurs, whereas, when $\sigma_{z z}^{\infty}<0$, a curvingperpendicular geometry occurs. Based on these considerations and using equations (3-1) and (3-2), the general formulations for the curving-parallel geometry case are

$$
\sigma_{y}^{\infty}=\frac{\sigma_{2}^{\infty}+\sigma_{3}^{\infty}}{2}-\frac{\sigma_{2}^{\infty}-\sigma_{3}^{\infty}}{2} \cos 2 \varsigma<0,
$$

and

$$
\sigma_{z z}^{\infty}=\frac{\sigma_{2}^{\infty}+\sigma_{3}^{\infty}}{2}+\frac{\sigma_{2}^{\infty}-\sigma_{3}^{\infty}}{2} \cos 2 \varsigma>0
$$

Combining these two inequalities yields

$$
-(1+\cos 2 \varsigma) /(1-\cos 2 \varsigma)<\sigma_{2}^{\infty} / \sigma_{3}^{\infty}<-(1-\cos 2 \varsigma) /(1+\cos 2 \varsigma) .
$$

Simple inspection of equation (3-8) reveals that it is only valid when $0^{\circ}<\varsigma<45^{\circ}$, which means that curving-parallel geometries can occur only when the angle between the pre-existing joints and the main trend of the cross joints is less than $45^{\circ}$.

Following the same steps, the conditions for the curvingperpendicular case are

$$
\begin{aligned}
& \sigma_{y y}^{\infty}=\frac{\sigma_{2}^{\infty}+\sigma_{3}^{\infty}}{2}-\frac{\sigma_{2}^{\infty}-\sigma_{3}^{\infty}}{2} \cos 2 \varsigma<0, \\
& \sigma_{z z}^{\infty}=\frac{\sigma_{2}^{\infty}+\sigma_{3}^{\infty}}{2}+\frac{\sigma_{2}^{\infty}-\sigma_{3}^{\infty}}{2} \cos 2 \varsigma<0,
\end{aligned}
$$

and

which lead to 


$$
\frac{\sigma_{2}^{\infty}}{\sigma_{3}^{\infty}}>-\frac{1-\cos 2 \varsigma}{1+\cos 2 \varsigma}, \quad\left(0^{\circ}<\varsigma<45^{\circ}\right)
$$

and

$$
\frac{\sigma_{2}^{\infty}}{\sigma_{3}^{\infty}}>-\frac{1+\cos 2 \varsigma}{1-\cos 2 \varsigma} . \quad\left(45^{\circ}<\varsigma<90^{\circ}\right)
$$

Combining (3-11) and (3-12), we have

$$
\frac{\sigma_{2}^{\infty}}{\sigma_{3}^{\infty}}>-\frac{1-|\cos 2 \varsigma|}{1+|\cos 2 \varsigma|}, \quad\left(0^{\circ}<\varsigma<90^{\circ}\right)
$$

Considering the fact that $\sigma_{2}^{\infty} \geq \sigma_{3}^{\infty}$ and $\sigma_{3}^{\infty}<0$, then

$$
\sigma_{2}^{\infty} / \sigma_{3}^{\infty} \leq 1
$$

Therefore, the conditions for achieving a curving-perpendicular geometry in the open case are

$$
-(1-|\cos 2 \varsigma|) /(1+|\cos 2 \varsigma|)<\sigma_{2}^{\infty} / \sigma_{3}^{\infty}<1 .
$$

Dyer (1988) specifically looked at the situation where $\varsigma=30^{\circ}$.

Substituting this value into (3-8) and (3-15), the remote stress ratio is $-3<\sigma_{2}^{\infty} / \sigma_{3}^{\infty}<-1 / 3$ for curving-parallel geometries, and $-1 / 3<\sigma_{2}^{\infty} / \sigma_{3}^{\infty}<1$ for curving perpendicular geometries. Thus my results are in complete agree with Dyer's conclusions. The results from (3-8) and (3-15) are summarized as the open cases in Table 3-2. 
Table 3-2. Classification of cross joint geometries. In this table, $\varsigma$ represents the take off angle, $\sigma_{2}^{\infty} / \sigma_{3}^{\infty}=\eta$ is the remote principal stress ratio, $C_{0}^{*}=[(\eta-1) \sin 2 \varsigma] /[\eta(1-\cos 2 \varsigma)+(1+\cos 2 \varsigma)]$ is the critical frictional coefficient, $C_{0}$ is the coefficient of friction for the pre-existing joint. $S=-(1+|\cos 2 \varsigma|) /(1-|\cos 2 \varsigma|), T=-(1-|\cos 2 \varsigma|) /(1+|\cos 2 \varsigma|)$.

\begin{tabular}{|c|c|c|c|}
\hline \multirow[b]{2}{*}{$0^{\circ}<\varsigma<45^{\circ}$} & $\frac{\sigma_{2}^{\infty}}{\sigma_{3}^{\infty}}<\mathrm{S}$ & $\mathrm{S}<\frac{\sigma_{2}^{\infty}}{\sigma_{3}^{\infty}}<\mathrm{T}$ & $\mathrm{T}<\frac{\sigma_{2}^{-}}{\sigma_{3}^{\alpha}}<1$ \\
\hline & $\begin{array}{c}\text { CLOSED CASE \#1 } \\
\sigma_{y}^{\infty}>0, \sigma_{z}^{\infty}>0 \\
\text { quasi-curving } \\
\text { parallel }\end{array}$ & $\begin{array}{c}\text { OPEN CASE \# 1 } \\
\sigma_{y}^{\infty}<0, \sigma_{z}^{\infty}>0 \\
\text { curving-parallel }\end{array}$ & $\begin{array}{c}\text { OPEN CASE \#2 } \\
\sigma_{y}^{\infty}<0, \sigma_{z}^{\infty}<0 \\
\text { curving- } \\
\text { perpendicular }\end{array}$ \\
\hline \multirow[b]{3}{*}{$45^{\circ}<\varsigma<90$} & $\begin{array}{c}\text { CLOSED CASE \#2 } \\
\sigma_{r}^{\infty}>0, \sigma_{z}^{\infty}>0\end{array}$ & $\begin{array}{c}\text { CLOSED CASE \#3 } \\
\sigma_{v}^{\infty}>0, \sigma_{z}^{\infty}<0\end{array}$ & \multirow{3}{*}{$\begin{array}{c}\text { OPEN CASE \#3 } \\
\sigma_{y}^{\infty}<0, \sigma_{z}^{\infty}<0 \\
\text { curving- } \\
\text { perpendicular }\end{array}$} \\
\hline & $\begin{array}{c}\text { Closed Case \#2a } \\
C_{0} \leq C_{0}^{*} \\
\text { unlocked } \\
\text { pre-existing joint } \\
\text { quasi-curving- } \\
\text { perpendicular }\end{array}$ & $\begin{array}{l}\text { Closed Case \#3a } \\
C_{0} \leq C_{0}^{*} \\
\text { unlocked } \\
\text { pre-existing joint } \\
\text { quasi-curving- } \\
\text { perpendicular }\end{array}$ & \\
\hline & $\begin{array}{l}\text { Closed Case \#2b } \\
C_{0}>C_{0}^{*} \\
\text { locked } \\
\text { pre-existing joint } \\
\text { non-curving }\end{array}$ & $\begin{array}{l}\text { Closed Case \#3b } \\
\qquad C_{0}>C_{0}^{*} \\
\text { locked } \\
\text { pre-existing joint } \\
\text { non-curving }\end{array}$ & \\
\hline
\end{tabular}




\section{(2). Local stresses adjacent to the pre-existing joint}

In order to calculate the cross joint paths using the assumption that cross joint traces follow the trajectory of the maximum principal stress in the $x=0$ plane (refer to Fig. 3-2), we first need to formulate the boundary conditions, and then calculate the stress components around the pre-existing joints. By setting $x=0$ for the general solution, we can obtain the stresses in the $x=0$ plane and thus calculate the principal stresses in the $x=0$ plane.

An open pre-existing joint means the joint faces are traction free. Therefore, the boundary conditions on the pre-existing joint surfaces are

$$
\text { at } y=0,|x|<\mathrm{c}: \quad \sigma_{y y}=\sigma_{y z}=\sigma_{y x}=0 \text {, }
$$

i.e., the normal stress $\left(\sigma_{y y}\right)$ and the shear stresses $\left(\sigma_{y z}, \sigma_{y x}\right)$ on the preexisting joint are zero.

According to the theoretical model (Fig. 3-2), we can formulate the remote boundary conditions as

$$
\text { at }\left(x^{2}+y^{2}\right)^{1 / 2} \rightarrow \infty: \quad \begin{aligned}
& \sigma_{x x}=\sigma_{x x}^{\infty}, \\
& \sigma_{y y}=\sigma_{y y}^{\infty}, \\
& \sigma_{z z}=\sigma_{z z}^{\infty}, \\
& \sigma_{y z}=\sigma_{y z}^{\infty}, \\
& \sigma_{x y}=0, \\
& \sigma_{x z}=0,
\end{aligned}
$$




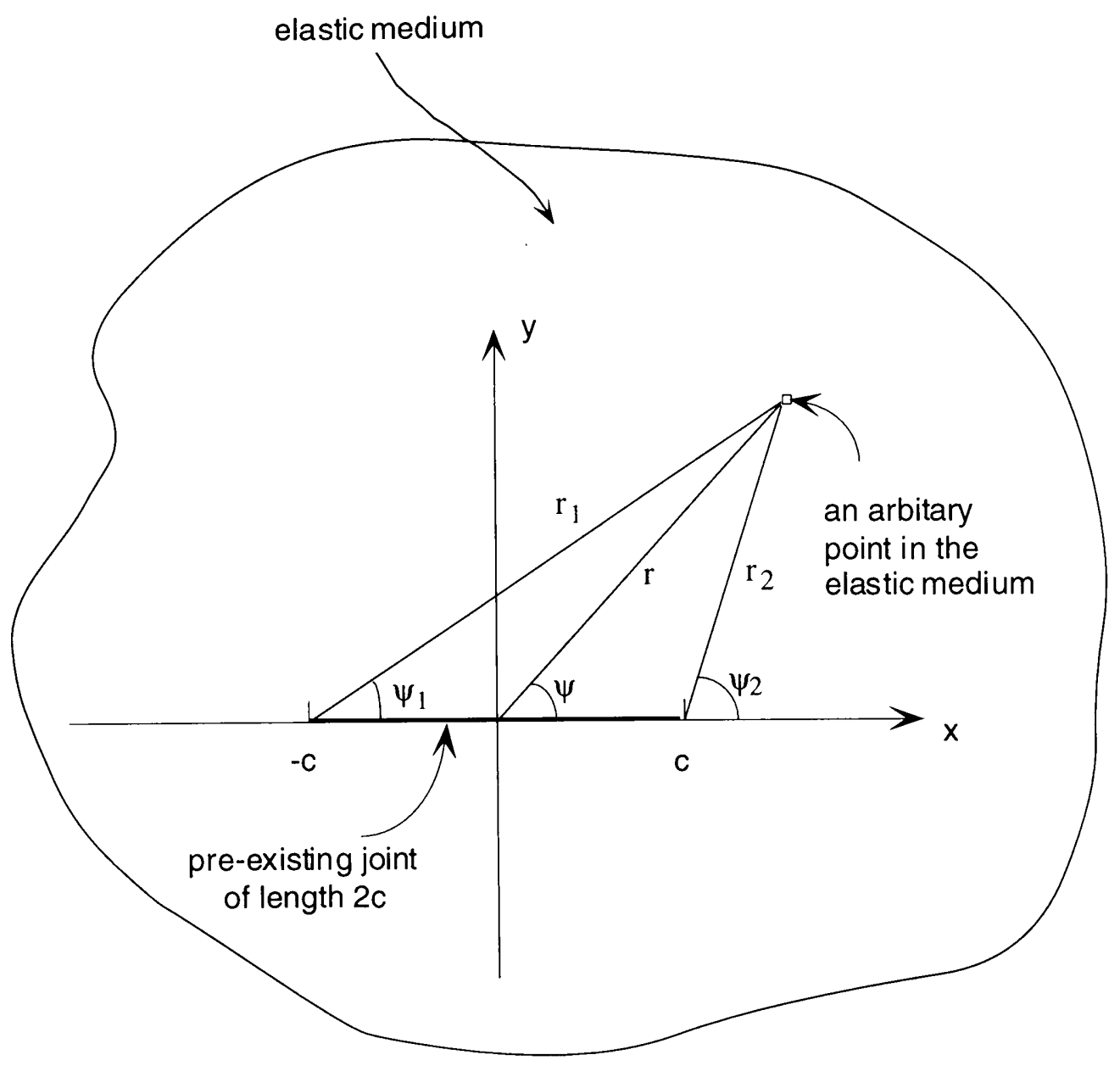

Fig. 3-3. Tripolar coordinate system used in deriving local stresses. Redrawn from Pollard and Segall (1987). 
where $\sigma_{x x}, \sigma_{y y}, \sigma_{z z}, \sigma_{y z}, \sigma_{x y}$ and $\sigma_{x z}$ are local normal and shear stresses.

Using the above boundary conditions, we can employ Pollard and Segall's (1987) derivation based on elastic fracture mechanics theory to determine the stress components at a specified point adjacent to the preexisting joint (see Fig. 3-3). These are

$$
\begin{aligned}
& \sigma_{y y}=\sigma_{y y}^{\infty}\left[\frac{r}{R} \cos (\psi-\Psi)+\frac{c^{2} r}{R^{3}} \sin \psi \sin 3 \Psi\right], \\
& \sigma_{x x}=\sigma_{x x}^{\infty}+\sigma_{y y}^{\infty}\left[\frac{r}{R} \cos (\psi-\Psi)-1-\frac{c^{2} r}{R^{3}} \sin \psi \sin 3 \Psi\right], \\
& \sigma_{y z}=\sigma_{y z}^{\infty}\left[\frac{r}{R} \cos (\psi-\Psi)\right], \\
& \sigma_{z z}=\sigma_{z z}^{\infty} .
\end{aligned}
$$

Here $c$ is the half height of the pre-existing joint (see Fig. 3-2), $r=\sqrt{x^{2}+y^{2}}, r_{1}=\sqrt{(x+c)^{2}+y^{2}}, r_{2}=\sqrt{(x-c)^{2}+y^{2}}, R=\sqrt{r_{1} r_{2}}$, $\Psi=\left(\psi_{1}+\psi_{2}\right) / 2, \psi, r_{1}, r_{2}, \psi_{1}$ and $\psi_{2}$ are all shown in Fig. 3-3.

To study cross joint propagation paths in the $x=0$ plane (Fig. 3-2), only $\sigma_{y y}, \sigma_{y z}$ and $\sigma_{z z}$ are useful. By letting $x=0$ in equations (3-16), (3-18) and (3-19), the stresses in the $x=0$ plane become

$$
\sigma_{y}=\frac{\sigma_{y y}^{\infty} y^{3}}{\left(y^{2}+c^{2}\right)^{3 / 2}}
$$




$$
\begin{aligned}
& \sigma_{y z}=\frac{\sigma_{y z}^{\infty} y}{\left(y^{2}+c^{2}\right)^{1 / 2}}, \\
& \sigma_{z z}=\sigma_{z z}^{\infty} .
\end{aligned}
$$

These equations provide expressions for local stresses as a function of distance from the pre-existing joint and remote stresses.

\section{(3). Cross joint trajectories}

Let $\theta$ represent the angle from the direction of local maximum horizontal principal stress to the $z$ axis (Fig. 3-4). According to the theory of elastic mechanics (Timoshenko and Goodier, 1970), we have

$$
\tan 2 \theta=2 \sigma_{y z} /\left(\sigma_{z z}-\sigma_{y y}\right)
$$

By substituting (3-1), (3-2), (3-3), (3-20), (3-21) and (3-22) into (323), we have

$$
\tan 2 \theta=\frac{2\left(\sigma_{2}^{\infty}-\sigma_{3}^{\infty}\right)\left[\frac{y}{\left(y^{2}+c^{2}\right)^{1 / 2}}\right] \sin 2 \varsigma}{\left(\sigma_{2}^{\infty}+\sigma_{3}^{\infty}\right)+\left(\sigma_{2}^{\infty}-\sigma_{3}^{\infty}\right) \cos 2 \varsigma-\left[\left(\sigma_{2}^{\infty}+\sigma_{3}^{\infty}\right)-\left(\sigma_{2}^{\infty}-\sigma_{3}^{\infty}\right) \cos 2 \varsigma\right] \frac{y^{3}}{\left(y^{2}+c^{2}\right)^{3 / 2}}} .
$$

This equation gives the angle between the local maximum principal stress and the pre-existing joint ( $z$-axis). Assuming that cross joints always propagate in the direction of local maximum principal stress, the angle $\theta$ 


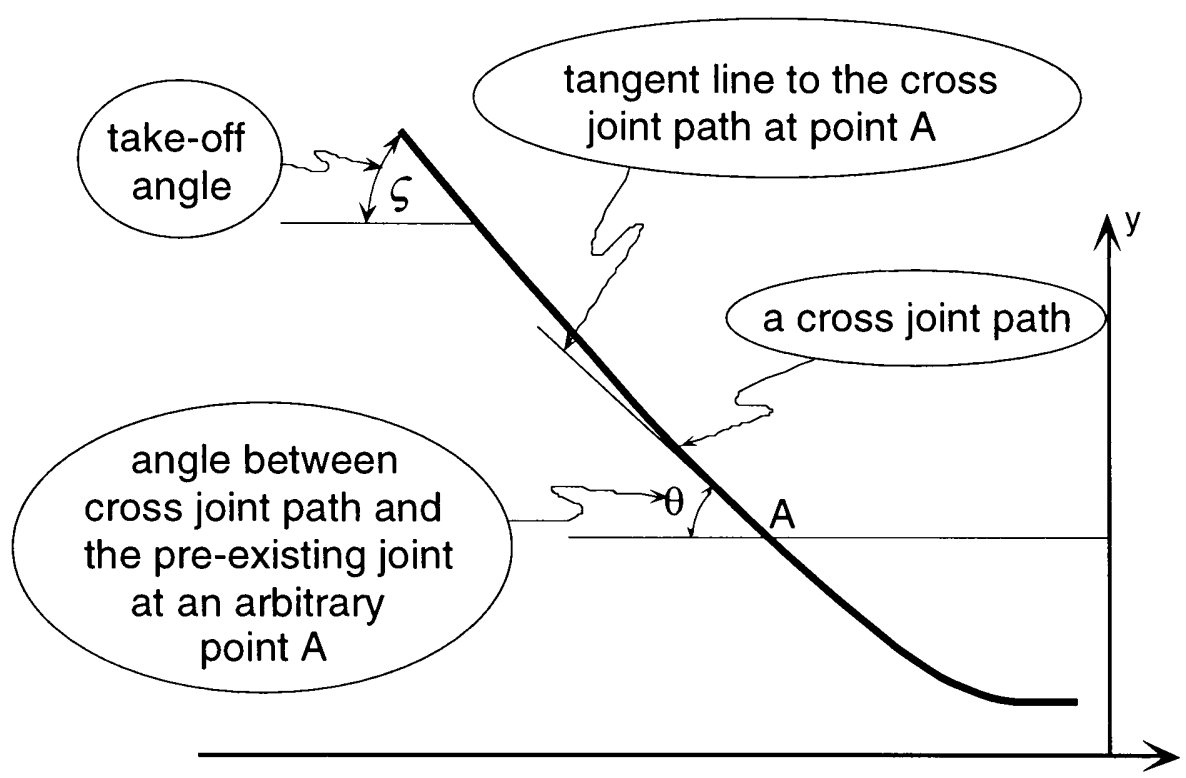

$z$ axis (collinear with the pre-existing fracture)

Fig. 3-4. Schematic diagram showing the definitions of take off angle ( $\varsigma$ ) and local cross joint angle $(\theta)$. 
represents the angle between the pre-existing joint and the tangent to the cross joint path (Fig. 3-4). Therefore, equation (3-24) describes the change of $\theta$ as a function of normalized distance from the pre-existing joint $(y / c)$, and thus delineates the trajectory for cross joint growth. I refer to $\theta$ as the "local cross joint angle".

As two examples, I plotted $\theta$ as a function of the normalized distance from the pre-existing joint $(y / c)$ in Fig. 3-5 and Fig. 3-7, assuming a takeoff angle of $30^{\circ}$ and $60^{\circ}$, respectively, and under different remote principal stress ratios $\left(\sigma_{2}^{\infty} / \sigma_{3}^{\infty}\right)$. Figures 3-6 and 3-8 show the physical views of cross joint paths corresponding to Fig. 3-5 and 3-7.

\section{CLOSED PRE-EXISTING CRACK CASE}

Now let us turn our attention to the other major category of preexisting joints, namely the closed case. In dealing with the closed case, the same procedures used in discussing the open case are not applicable because in the open pre-existing joint case, curving-parallel and curvingperpendicular geometry classification is based solely on the signs of the remote normal stresses (i.e., $\sigma_{y y}^{\infty}$ and $\sigma_{z z}^{\infty}$ ). In the closed case, however, cross joint geometry classification cannot be determined before obtaining the local cross joint angle. In order to obtain the local cross joint angle, one must derive stresses in the area adjacent to the cross joint. Therefore, this section will follow a different three-step procedure. The first step is to derive the stresses adjacent to the pre-existing joint. Then, a general formulation of the local cross joint angle is made based on these stress 


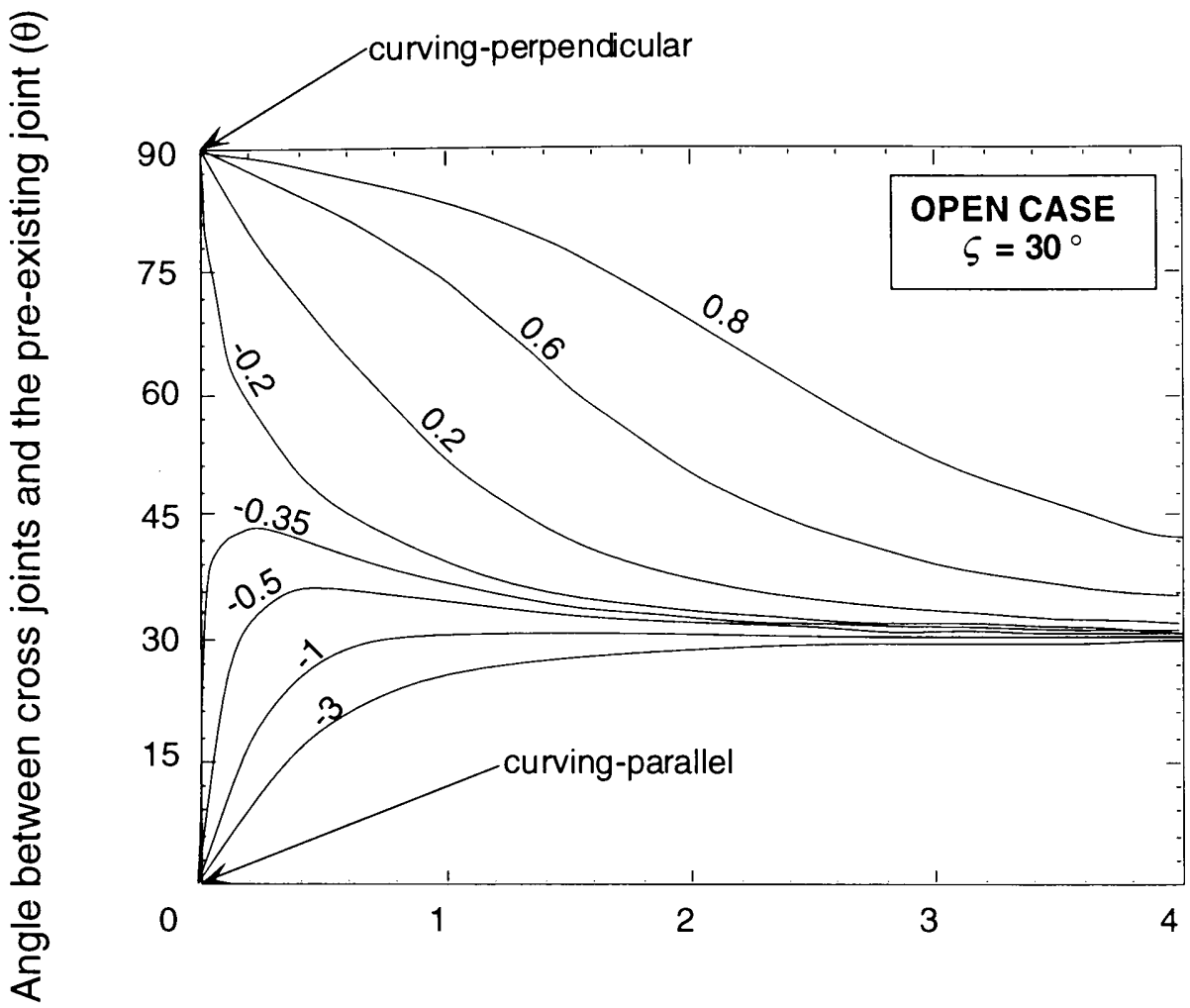

Normalized distance from the pre-existing joint $(y / c)$

Fig. 3-5. Relationships between local cross joint angle $(\theta)$ and the normalized distance from the pre-existing joint $(y / c)$ for different remote principal stress ratios $(\eta)$, a take off angle $(\varsigma)$ of $30^{\circ}$, and under the conditions for an open pre-existing joint. The number next to each curve indicates the remote principal stress ratio. Note that when $-3<\eta<-1 / 3$ curving-parallel geometry occurs, corresponding to Open Case \#1 in Table 3-2. When $-1 / 3<\eta<1$ curving-perpendicular geometry occurs, corresponding to Open Case \#2 in Table 3-2. 
OPEN CASE $\varsigma=30^{\circ}$
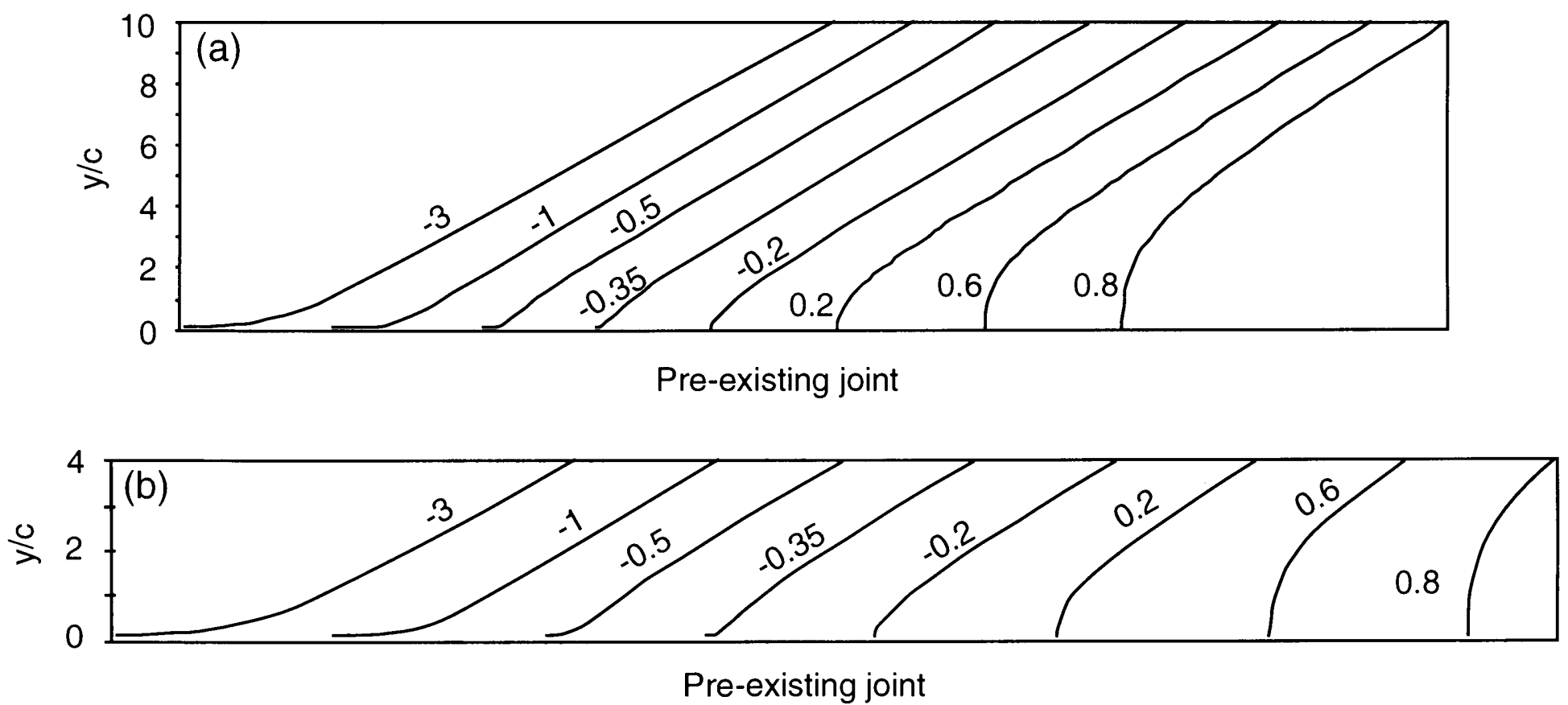

Fig. 3-6. Physical views of cross joint propagation paths corresponding to Fig. 3-5. The takeoff angle is $30^{\circ}$. The number next to each curve (in both a and b) indicates the remote principal stress ratio. (a) Small scale. (b) Large scale showing closed-up view of intersection between cross joint and pre-existing joint. 


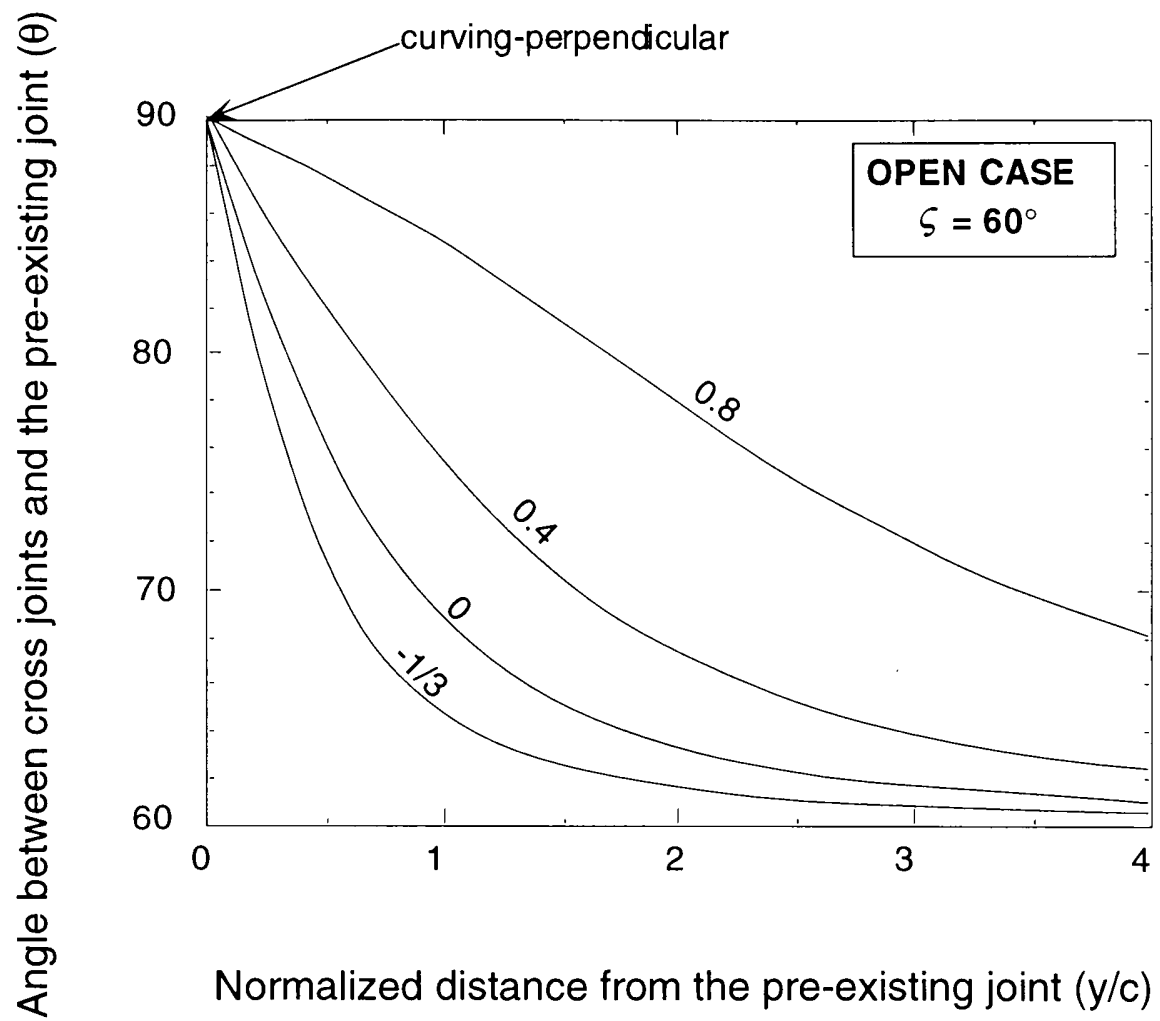

Fig. 3-7. Relationships between local cross joint angle $(\theta)$ and the normalized distance from the pre-existing joint $(y / c)$ for different remote principal stress ratios $(\eta)$ a take off angle $(\varsigma)$ of $60^{\circ}$, and under the conditions for an open pre-existing joint. The number next to each curve indicates the remote principal stress ratio. Note that only curving-perpendicular geometry occurs in this case, corresponding to Open Case \#3 in Table 3-2. 


\section{OPEN CASE $\varsigma=60^{\circ}$}
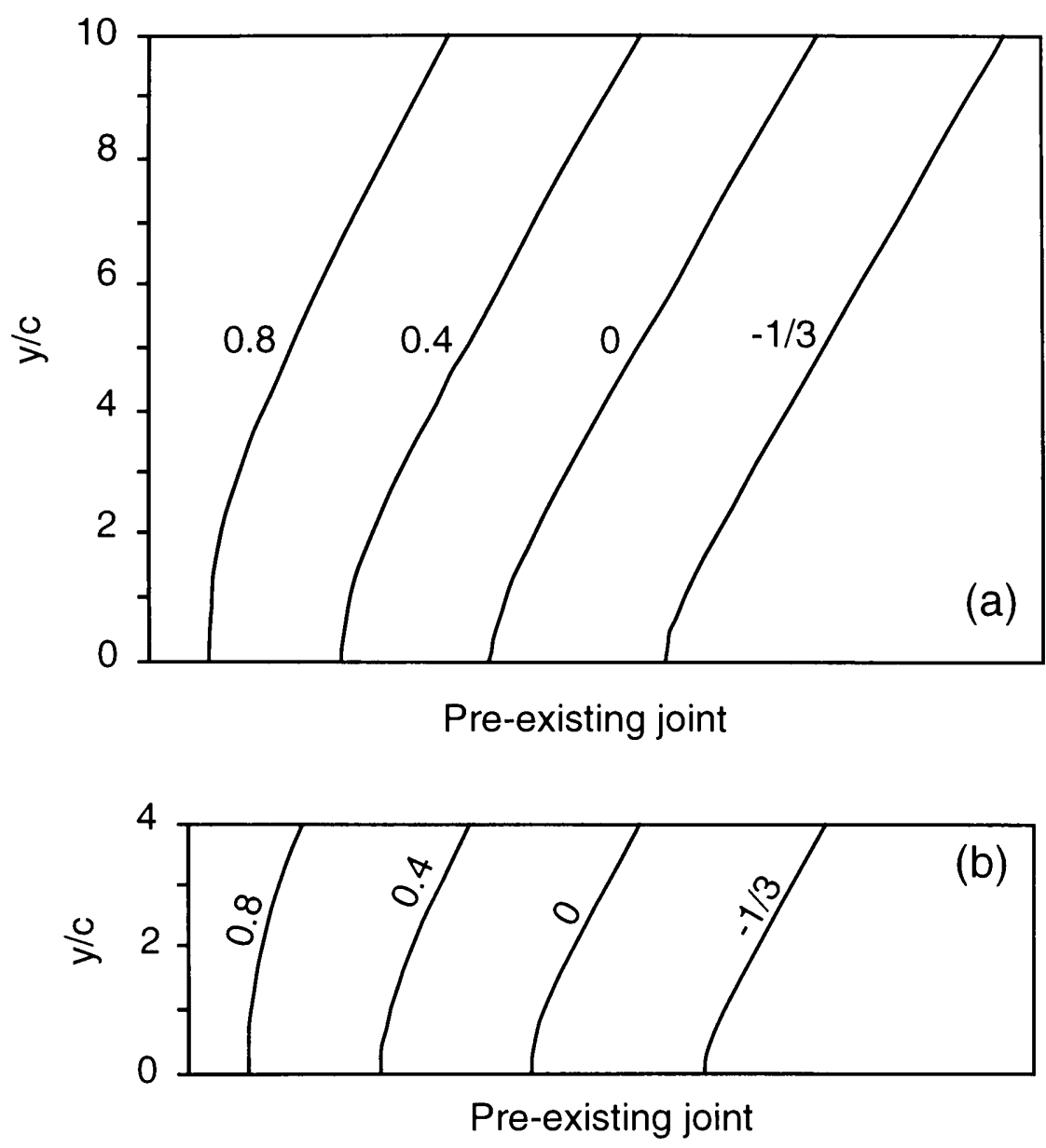

Fig. 3-8. Physical views of cross joint propagation paths corresponding to Fig. 3-7. The takeoff angle $(\varsigma)$ is $60^{\circ}$. The number next to each curve (in both a and b) indicates the remote principal stress ratio. (a) Small scale. (b) Large scale showing close-up view of intersection between cross joint and pre-existing joint. 
solutions, and a general cross joint geometry classification is constructed based on the possible values of the local cross joint angle. The third step provides a detailed cross joint geometry classification and plots theoretical cross joint trajectories considering the friction along the pre-existing joint.

\section{(1). Local stresses adjacent to the pre-existing joint}

In the closed pre-existing joint case, the pre-existing joint faces are not traction free, which means the shear stresses and the normal stress along the pre-existing joint surfaces are not zero. Here, I consider a simple case in which the normal stress acting on the pre-existing surface $\left(\sigma_{v v}\right)$ is not zero, and equals to the remote normal stress in the same direction $\left(\sigma_{v \prime}^{\infty}\right)$. The shear stress in the $z$ direction $\left(\sigma_{y z}\right)$ is not zero, and it is a fraction of the remote shear stress of the same direction $\left(\sigma_{v z}^{\infty}\right)$, but the shear stress in the $x$ direction $\left(\sigma_{y x}\right)$ is zero. Therefore the boundary conditions on the preexisting joint surfaces can be formulated as

$$
\text { at } y=0,|x|<\mathrm{c}: \quad \begin{aligned}
& \sigma_{y y}=\sigma_{y y}^{\infty}, \\
& \sigma_{y z}=s \sigma_{y z}^{\infty}, \\
& \sigma_{y x}=0,
\end{aligned}
$$

where $s$ is called the shear stress ratio, i.e., the ratio of the shear stress on the pre-existing joint ( $\sigma_{y z}$ at $y=0$ and $|x|<c$ ) to the remote shear stress $\left(\sigma_{y z}^{\infty}\right)$, and $0 \leq s \leq 1$. From its definition, we know that $s=0$ corresponds to a perfectly lubricated pre-existing joint, while $s=1$ represents a locked preexisting joint, i.e., the two walls of the joint are completely welded 
together. Using a Byerlee (1978) type friction law, we can express $s$ as $s=C_{0} \sigma_{y y}^{\infty} / \sigma_{y z}^{\infty}$, provided that the right side of this equation falls within the range of 0 to 1 , where $C_{0}$ is the coefficient of friction.

The remote stresses for the closed case are the same as in the open case (Fig. 3-2), which are

$$
\text { at } \begin{aligned}
\left(x^{2}+y^{2}\right)^{1 / 2} \rightarrow \infty: & \sigma_{x x}=\sigma_{x x}^{\infty}, \\
\sigma_{y y} & =\sigma_{y y}^{\infty}, \\
\sigma_{z z} & =\sigma_{z z}^{\infty}, \\
\sigma_{y z} & =\sigma_{y z}^{\infty}, \\
\sigma_{x y} & =\sigma_{x z}=0 .
\end{aligned}
$$

Again according to Pollard and Segall (1987), we obtain the general solutions for the non-zero stresses around the pre-existing joint, which are

$$
\begin{aligned}
& \sigma_{x x}=\sigma_{x x}^{\infty}, \\
& \sigma_{y y}=\sigma_{y y}^{\infty}, \\
& \sigma_{z z}=\sigma_{z z}^{\infty}, \\
& \sigma_{y z}=s \sigma_{y z}^{\infty}+(1-s) \sigma_{y z}^{\infty}\left[\frac{r}{R} \cos (\psi-\Psi)\right] .
\end{aligned}
$$

In the $x=0$ plane, we have 


$$
\begin{aligned}
& \sigma_{y y}=\sigma_{y y}^{\infty}, \\
& \sigma_{z z}=\sigma_{z z}^{\infty}, \\
& \sigma_{y z}=s \sigma_{y z}^{\infty}+(1-s) \sigma_{y z}^{\infty} \frac{y}{\left(y^{2}+c^{2}\right)^{1 / 2}} .
\end{aligned}
$$

These equations give the local stresses adjacent to the pre-existing joint.

\section{(2). General formulation of local cross joint angle and cross joint geometry classification}

As I did in discussing the open case, local cross joint angle $(\theta)$ can be obtained using the relationship between $\theta$ and local stresses adjacent to the pre-existing joint as expressed by equation (3-23).

Substituting (3-29) (3-30) (3-31) (3-1) (3-2) (3-3) into (3-23), we have

$$
\tan 2 \theta=\left[s+(1-s) \frac{y}{\left(y^{2}+c^{2}\right)^{1 / 2}}\right] \tan 2 \varsigma .
$$

Equation (3-32) shows the relation between the instantaneous angle of the cross joint $(\theta)$ and normalized distance from the pre-existing joint $(y / c)$ for different shear stress ratios $(s)$ and takeoff angles $(\varsigma)$. In order to classify cross joint geometry in the closed case, let us consider the value 
of $\tan 2 \theta$ when $y=0$, which marks the intersection point of the cross joint with the pre-existing joint. Substituting $y=0$ into equation (3-32), we have

$$
\tan 2 \theta=s \tan 2 \varsigma .
$$

Considering the condition for each closed pre-existing joint case in Table 3-1, we can construct Table 3-2 based on the following subdivisions: (i). For Closed Case \#1, since $0 \leq s \leq 1$, and $\tan 2 \varsigma \geq 0$ for $0^{\circ} \leq \varsigma \leq 45^{\circ}$, we have, $\tan 2 \theta \geq 0$ or $0^{\circ} \leq \theta \leq 45^{\circ}$ at $y=0$. In other words, cross joints are not necessarily aligned parallel or perpendicular to the pre-existing joints at the point of intersection. Rather, the local cross joint angle lies between $0^{\circ}$ and $45^{\circ}$. Hence, I label this kind of geometry as "quasi-curving-parallel" geometry. The two end members of this case correspond to $s=0$ and $s=$ 1. For $s=0, \tan 2 \theta=0$ at $y=0$, which corresponds to $\theta=0^{\circ}$ and a curving-parallel geometry. On the other hand, when $s=1$, i.e., the preexisting joint is locked, we have $\tan 2 \theta=\tan 2 \varsigma$. Thus, $\theta=\varsigma$, which means the cross joint path does not deviate from its original take-off angle as it approaches the pre-existing joint.

(ii). For Closed Cases \#2 and \#3, we have $\tan 2 \theta \leq 0$, or $45^{\circ} \leq \theta \leq 90^{\circ}$ at $y=0$, based on the conditions of $0 \leq s \leq 1$ and $\tan 2 \varsigma \leq 0$ when $45^{\circ} \leq \varsigma \leq 90^{\circ}$. I refer to this geometry as "quasi-curving-perpendicular" geometry. Again, $s=0$ and $s=1$ represent two end members in these cases. When $s=0$, purely curving-perpendicular geometry occurs, whereas $s=1$ implies that cross joints do not curve. 


\section{(3). Cross joint geometry and the coefficient of friction along pre-existing joint----detailed cross joint geometry classification}

In order to determine the relationships among $\theta, y / c$, take-off angle $(\varsigma)$ and remote principal stress ratio $\left(\sigma_{2}^{\infty} / \sigma_{3}^{\infty}\right)$, we need to substitute $s=C_{0} \sigma_{y y}^{\infty} / \sigma_{v z}^{\infty}$ into equation (3-32). Before proceeding, first let us discuss the meaning of the constraint $0 \leq s \leq 1$. Using $s=C_{0} \sigma_{y y}^{\infty} / \sigma_{y z}^{\infty}$, and equations (3-1) and (3-3), we have

$$
0 \leq \frac{C_{0}\left[\left(\sigma_{2}^{\infty}+\sigma_{3}^{\infty}\right)-\left(\sigma_{2}^{\infty}-\sigma_{3}^{\infty}\right) \cos 2 \varsigma\right]}{\left(\sigma_{2}^{\infty}-\sigma_{3}^{\infty}\right) \sin 2 \varsigma} \leq 1 .
$$

For convenience here I use $\eta$ to represent the remote principal stress ratio $\left(\sigma_{2}^{\infty} / \sigma_{3}^{\infty}\right)$. Rewriting equation (3-34), we have

$$
\eta \leq-\frac{1+\cos 2 \varsigma}{1-\cos 2 \varsigma},
$$

and

$$
C_{0} \leq \frac{(\eta-1) \sin 2 \varsigma}{\eta(1-\cos 2 \varsigma)+(1+\cos 2 \varsigma)} .
$$

From Table 3-1 we can see that for all the closed cases, equation (3-35) can be satisfied. Inequality (3-36) is the constraint on the coefficient of friction by $s \leq 1$. However, the coefficient of friction for most rocks is nearly a constant (Byerlee, 1978), and thus equation (3-36) should be used to determine whether or not the pre-existing joint is locked. If we express the right side of the equation as the critical value of the frictional coefficient $\left(C_{o}^{*}\right)$, 


$$
C_{0}^{*}=\frac{(\eta-1) \sin 2 \varsigma}{\eta(1-\cos 2 \varsigma)+(1+\cos 2 \varsigma)},
$$

we can say that when $C_{0} \leq C_{0}^{*}$, the pre-existing joint is not locked, and $s=C_{0} \sigma_{y y}^{\infty} / \sigma_{y z}^{\infty}$ is valid. However, when $C_{0}>C_{0}^{*}$, the pre-existing joint is locked, and $s=1$.

Now let us consider the three closed cases in Table 3-1 in detail. Under the conditions for Closed Case \#1 in Table 3-1, and using equation (3-37), we can show that $C_{0}^{*} \geq 1$. That means in this case there is no constraint on the frictional coefficient of the pre-existing joint because $C_{0}$ is always less than $C_{0}^{*}$, and consequently $s=C_{0} \sigma_{y y}^{\infty} / \sigma_{y z}^{\infty}$ is valid. Substituting $s=C_{0} \sigma_{y y}^{\infty} / \sigma_{y z}^{\infty}$, and equations (3-1) (3-2) and (3-3) into equation (3-32), we obtain

$\tan 2 \theta=\left[\frac{y}{\left(y^{2}+c^{2}\right)^{1 / 2}}+\left(1-\frac{y}{\left(y^{2}+c^{2}\right)^{1 / 2}}\right) C_{0} \frac{(\eta+1)-(\eta-1) \cos 2 \varsigma}{(\eta-1) \sin 2 \varsigma}\right] \tan 2 \varsigma$.

As an example, Fig. 3-9 shows the relation between $\theta$ and $y / c$ under different remote principal stress ratios $(\eta)$ using a takeoff angle $(\varsigma)$ of $30^{\circ}$ and a frictional coefficient of 0.6. Figure 3-10 shows the physical views of cross joint paths corresponding to Fig. 3-9.

For Closed Cases \#2 and \#3 in Table 3-1, in order to use equation (338) to calculate cross joint paths, we must first consider whether equation (3-36) can be satisfied. If $C_{0} \leq C_{0}^{*}$, the pre-existing joint is not locked and we can use equation (3-38) to plot the cross joint paths. However, if $C_{0}>C_{0}^{*}$, the pre-existing joint is locked and $s=1$. From equation (3-32) we 


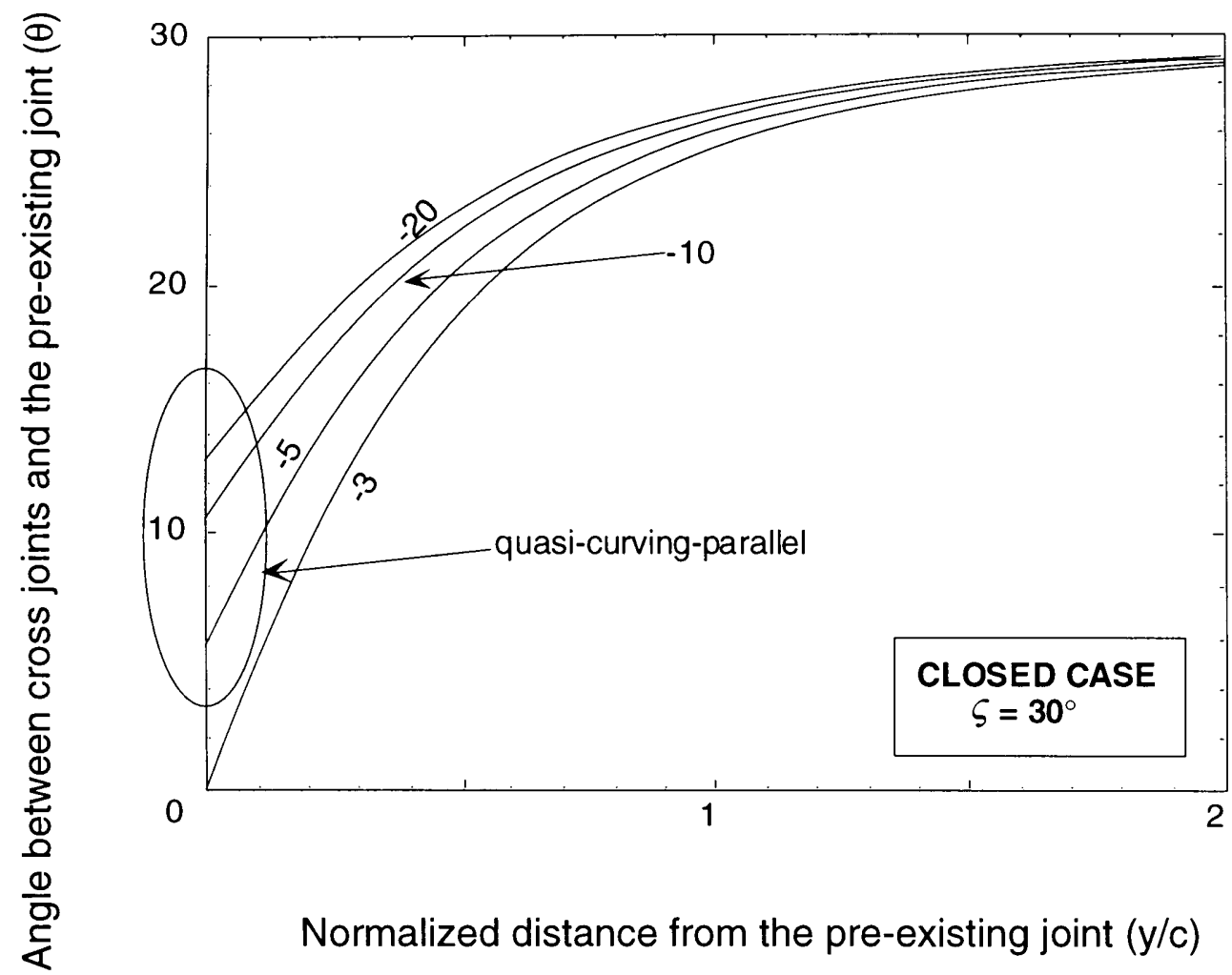

Fig. 3-9. Relationships between local cross joint angle $(\theta)$ and the normalized distance from the pre-existing joint $(y / c)$ for different remote principal stress ratios $(\eta)$, a take off angle $(\varsigma)$ of $30^{\circ}$, and under the conditions for a closed pre-existing joint. The number next to each curve indicates the remote principal stress ratio. The figure shows the quasi-curving-parallel case, which corresponding to Closed Case \#1 in Table 3-2. 
CLOSED CASE $\varsigma=30^{\circ}$
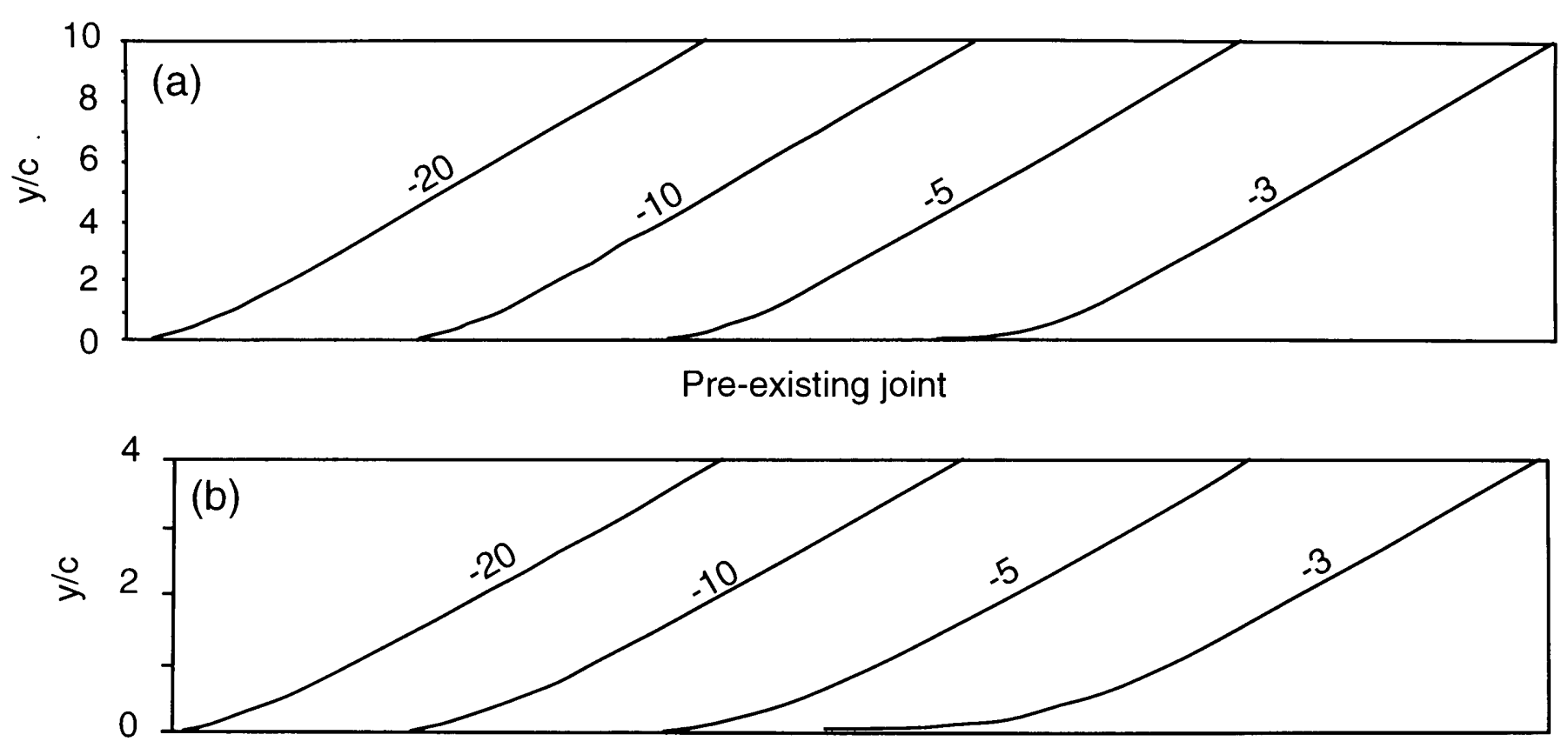

Pre-existing joint

Fig. 3-10. Physical views of cross joint propagation paths corresponding to Fig. 3-9. The takeoff angle is $30^{\circ}$. The number next to each curve (in both a and b) indicates the remote principal stress ratio. (a) Small scale. (b) Large scale showing close-up view of intersection between cross joint and pre-existing joint. 


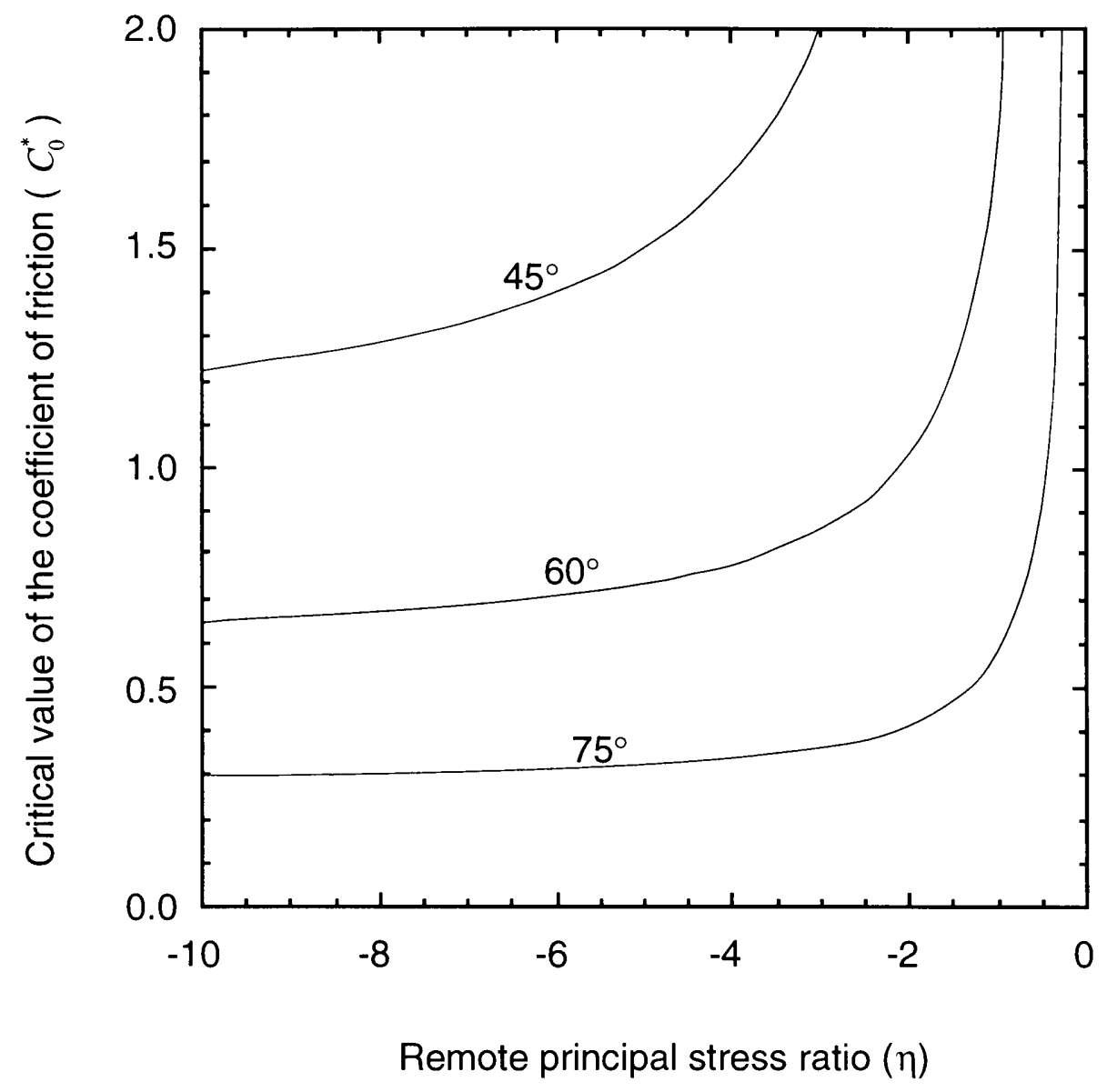

Fig. 3-11. Relations between the critical value of the coefficient of friction $\left(C_{0}^{*}\right)$ along the pre-existing joint and the remote principal stress ratio $(\eta)$. 
know that $\theta=\varsigma$ for $s=1$, which means that cross joint paths do not curve. Figure (3-11) shows the critical value of the coefficient of friction $\left(C_{0}^{*}\right)$ determined by equation (3-37) as a function of remote principal stress ratio $(\eta)$ at take-off angles $(\varsigma)$ of $45^{\circ}, 60^{\circ}$ and $75^{\circ}$. From this plot we can see that for a take-off angle of $60^{\circ}$, a frictional coefficient of 0.6 can satisfy $C_{0} \leq C_{0}^{*}$ at remote principal stress ratios greater than -10 and less than $-1 / 3$, which means the pre-existing joint is not locked. Therefore, we can use equation (3-38) to plot the relation between $\theta$ and the normalized distance from the pre-existing joint $(y / c)$, and to plot the cross joint paths which are shown in Fig. 3-12 and 3-13, respectively.

From the above analysis, we now have created subcategorizes for Closed Cases \#2 and \#3, namely the non-curving cases when $C_{0}>C_{0}^{*}$ (also called the locked-pre-existing joint cases) which are summarized in Table 32. Also, I diagrammatically show the cross joint geometry classification in Fig. 3-14. Figure 3-14a shows the frictionless cases $\left(C_{0}=0\right)$ along the preexisting joint, and Fig. $3-14 \mathrm{~b}$ shows the case of $C_{0}=0.6$. Table 3-2 and Fig. 3-14 represent an intermediate stage of cross joint classification. Incorporating the dimension of compressive zone, outlined in the following section, will result in a more detailed classification. 


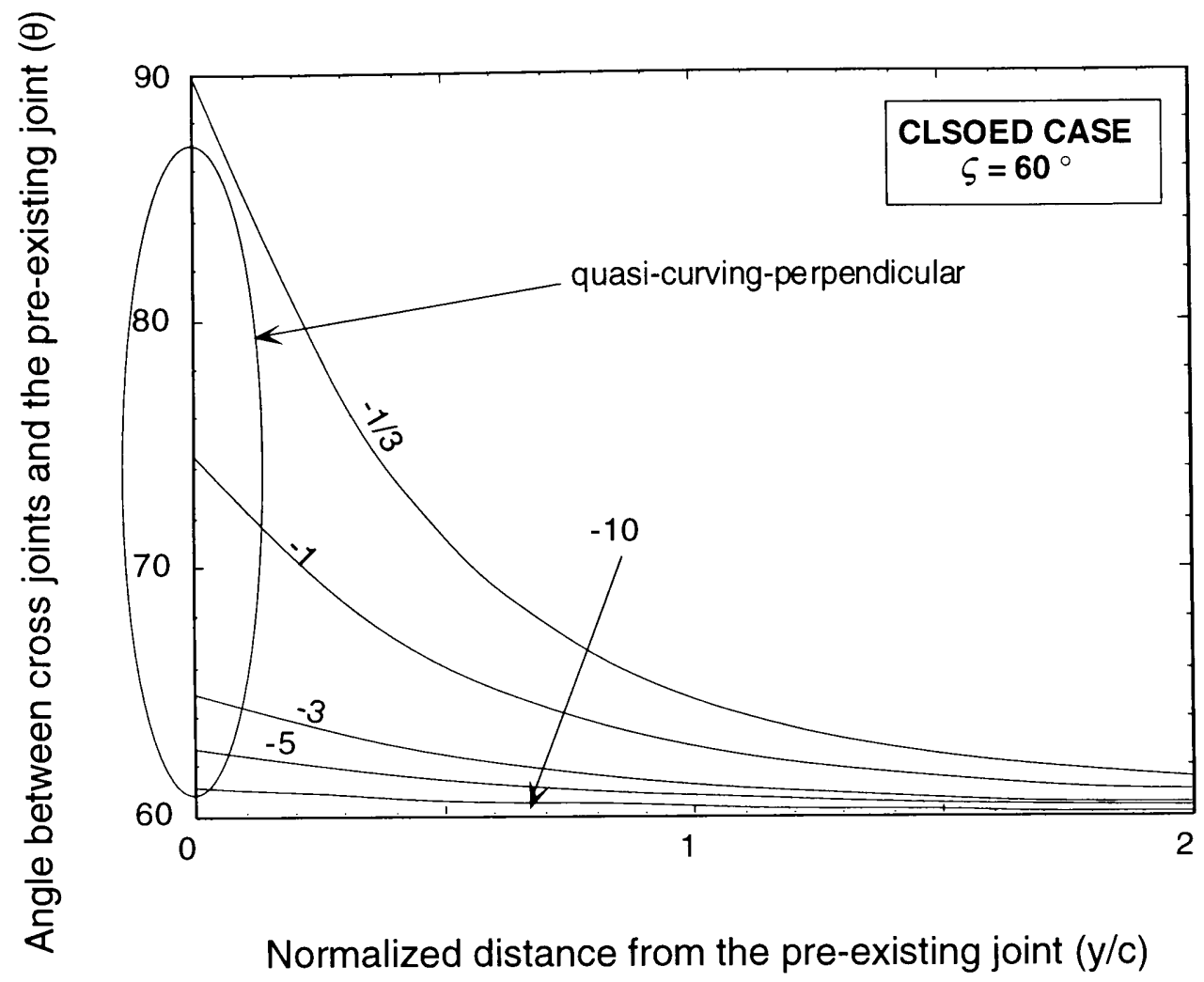

Fig. 3-12. Relationships between local cross joint angle ( $\theta)$ and the normalized distance from the pre-existing joint $(y / c)$ for different remote principal stress ratios $(\eta)$, a take off angle $(\varsigma)$ of $60^{\circ}$, and under the conditions for a closed pre-existing joint using a coefficient of friction along the pre-existing joint of 0.6. The number next to each curve indicates the remote principal stress ratio. The figure shows the quasi-curving-perpendicular case, corresponding to Closed Cases \#2a and \#3a in Table 3-2. 


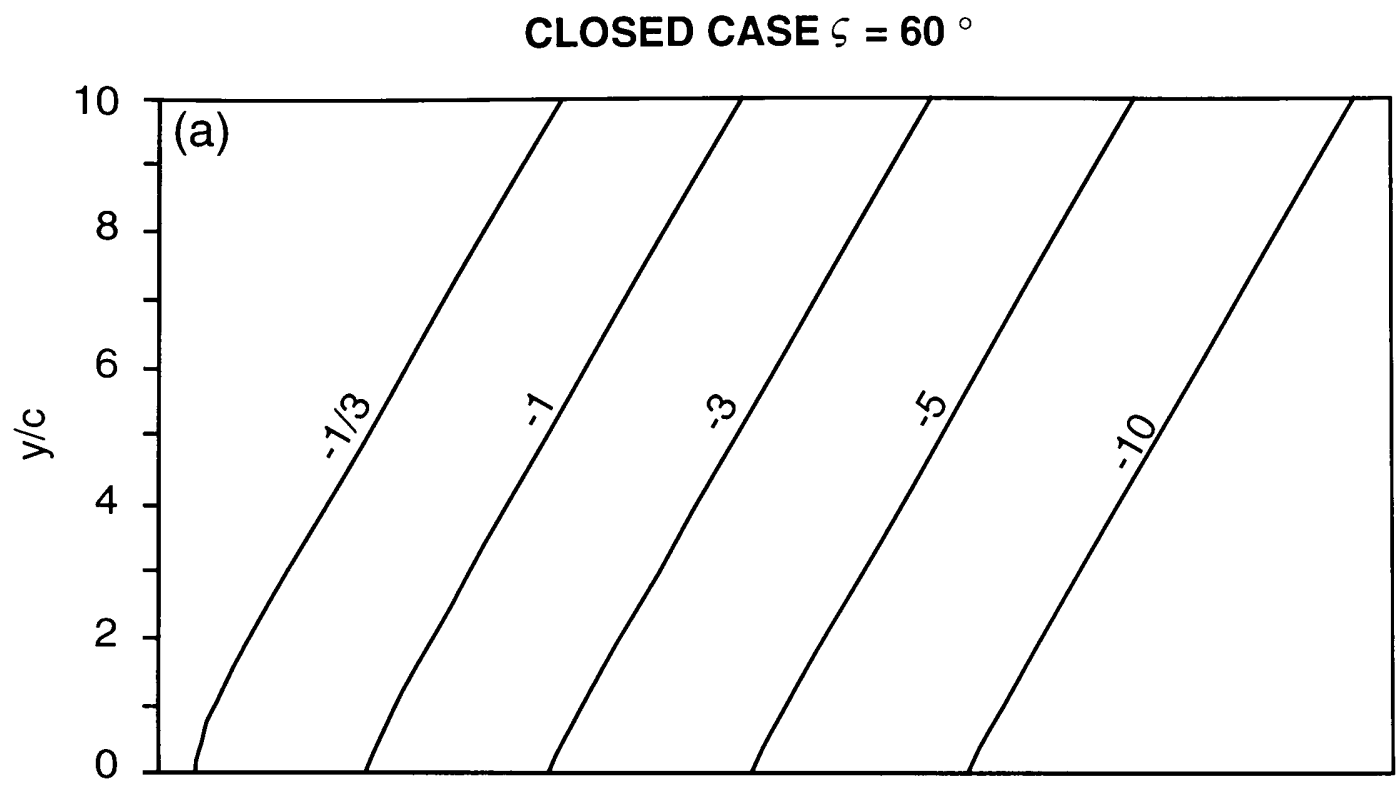

Pre-existing joint

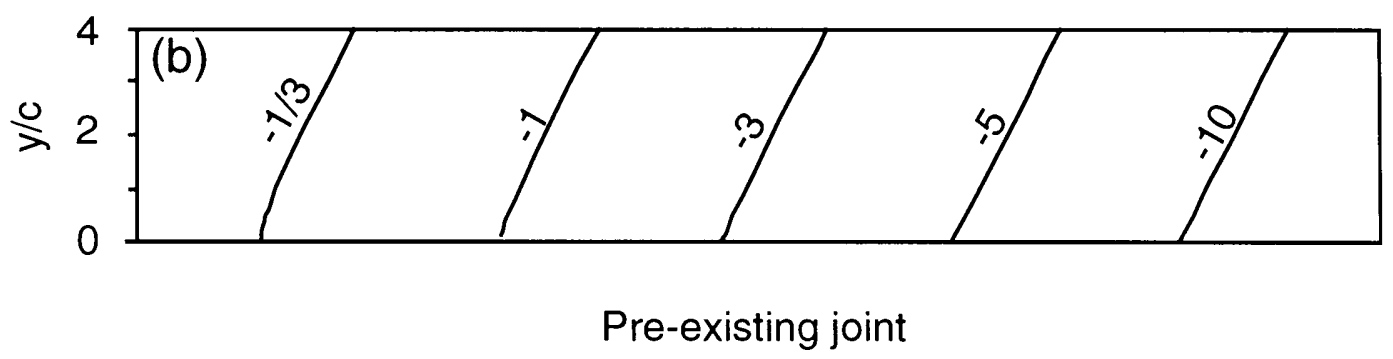

Fig. 3-13. Physical views of cross joint propagation paths corresponding to Fig. 3-12. The takeoff angle $(\varsigma)$ is $60^{\circ}$. The number next to each curve (in both $\mathrm{a}$ and $\mathrm{b}$ ) indicates the remote principal stress ratio. (a) Small scale. (b) Large scale showing closed-up view of intersection between cross joint and pre-existing joint. 

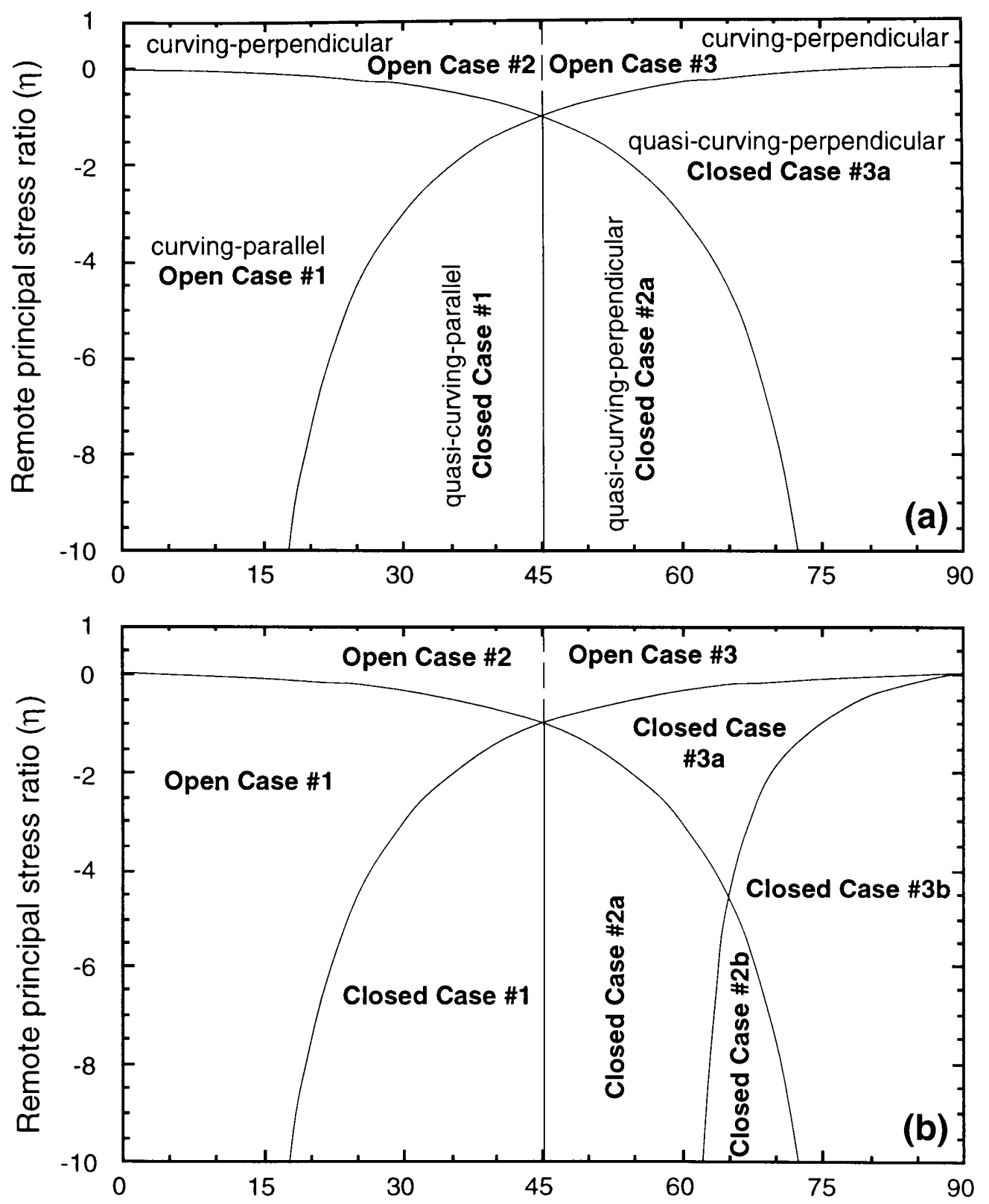

Take off angle $(\varsigma)$

Fig. 3-14. Cross joint geometry classification based on takeoff angle $(\varsigma)$ and remote principal stress ratio $(\eta)$. This figure is drawn according to the information provided in Table 3-2. (a) The case of a frictionless pre-existing joint. (b) The case when the coefficient of friction along the pre-existing joint $\left(C_{0}\right)$ is 0.6 . 


\section{COMPRESSIVE ZONE: CONSTRAINTS ON CROSS JOINT GROWTH}

Theoretically, a mode-I fracture cannot propagate into a region where the effective least principal stress is compressive. Therefore, the development of a compressive region (i.e., a zone where both $\sigma_{2}$ and $\sigma_{3}$ are compressive) adjacent to pre-existing joints would inhibit cross joint growth. Because localized zones of compression can in fact develop in fractured elastic media, it is important to determine the dimensions of such zones, and the boundary where $\sigma_{3}$ switches from tensile to compressive.

The latter may indicate the position where cross joints cease to grow. Dyer (1988) found that in open cases the dimension of the compressive zone is zero, implying that tips of cross joints remain in an effective tensile stress field throughout their growth. In contrast, for his closed pre-existing joint case, Dyer determined the extent of the compressive zone was approximately 0.6 times the half height of the pre-existing joint. In the following section I will provide a general expression for the dimension of the compressive zone and its related factors.

\section{OPEN PRE-EXISTING CRACK CASE}

From equations (3-1), (3-2), (3-3), (3-20), (3-21) and (3-22) we can calculate the magnitude of the local principal stresses in the open case, which are 


$$
\begin{gathered}
\sigma_{2,3}=(A+B \cos 2 \varsigma)+(A-B \cos 2 \varsigma) \frac{y^{3}}{\left(y^{2}+c^{2}\right)^{3 / 2}} \\
\left. \pm\left\{(A+B \cos 2 \varsigma)-(A-B \cos 2 \varsigma) \frac{y^{3}}{\left(y^{2}+c^{2}\right)^{3 / 2}}\right]^{2}+\left[\frac{2 B y \sin 2 \varsigma}{\left(y^{2}+c^{2}\right)^{1 / 2}}\right]^{2}\right\}^{1 / 2},
\end{gathered}
$$

where $A=\left(\sigma_{2}^{\infty}+\sigma_{3}^{\infty}\right) / 4$, and $B=\left(\sigma_{2}^{\infty}-\sigma_{3}^{\infty}\right) / 4$.

Let $t$ represent the normalized distance from the pre-existing joint $(y / c)$, and $\eta$ represent the remote principal stress ratio $\left(\sigma_{2}^{\infty} / \sigma_{3}^{\infty}\right)$. Considering $\sigma_{2} \geq \sigma_{3}$ and $\sigma_{3}^{\infty}<0$, we have

$$
\begin{gathered}
\sigma_{3}=\sigma_{3}^{\infty}\left[\left(\frac{\eta+1}{4}+\frac{\eta-1}{4} \cos 2 \varsigma\right)+\left(\frac{\eta+1}{4}-\frac{\eta-1}{4} \cos 2 \varsigma\right) \frac{t^{3}}{\left(t^{2}+1\right)^{3 / 2}}\right] \\
+\sigma_{3}^{\infty}\left\{\left[\left(\frac{\eta+1}{4}+\frac{\eta-1}{4} \cos 2 \varsigma\right)-\left(\frac{\eta+1}{4}-\frac{\eta-1}{4} \cos 2 \varsigma\right) \frac{t^{3}}{\left(t^{2}+1\right)^{3 / 2}}\right]^{2}+\left[\frac{(\eta-1) t \sin 2 \varsigma}{2\left(t^{2}+1\right)^{1 / 2}}\right]^{2}\right\}^{1 / 2} .
\end{gathered}
$$

From the above expression, we have

$$
\begin{gathered}
\sigma_{3} \leq \sigma_{3}^{\infty}\left[\left(\frac{\eta+1}{4}+\frac{\eta-1}{4} \cos 2 \varsigma\right)+\left(\frac{\eta+1}{4}-\frac{\eta-1}{4} \cos 2 \varsigma\right) \frac{t^{3}}{\left(t^{2}+1\right)^{3 / 2}}\right] \\
+\sigma_{3}^{\infty}\left|\left(\frac{\eta+1}{4}+\frac{\eta-1}{4} \cos 2 \varsigma\right)-\left(\frac{\eta+1}{4}-\frac{\eta-1}{4} \cos 2 \varsigma\right) \frac{t^{3}}{\left(t^{2}+1\right)^{3 / 2}}\right|,
\end{gathered}
$$

which is equivalent to 


$$
\begin{aligned}
& \sigma_{3} \leq \frac{\sigma_{3}^{\infty}}{4}\left[[\eta(1+\cos 2 \varsigma)+(1-\cos 2 \varsigma)]+[\eta(1-\cos 2 \varsigma)+(1+\cos 2 \varsigma)] \frac{t^{3}}{\left(t^{2}+1\right)^{3 / 2}}\right] \\
& +\frac{\sigma_{3}^{\infty}}{4}\left|[\eta(1+\cos 2 \varsigma)+(1-\cos 2 \varsigma)]-[\eta(1-\cos 2 \varsigma)+(1+\cos 2 \varsigma)] \frac{t^{3}}{\left(t^{2}+1\right)^{3 / 2}}\right| .
\end{aligned}
$$

Based on equation (3-42) and considering the conditions for the open pre-existing joint case in Table 3-1, which, in terms of $\eta$, are

$$
-\frac{1+\cos 2 \varsigma}{1-\cos 2 \varsigma}<\eta \leq 1 \text { and } 0^{\circ}<\varsigma<45^{\circ} \text { (Open Cases \#1 and \#2), }
$$

or

$$
\eta>-\frac{1-|\cos 2 \varsigma|}{1+|\cos 2 \varsigma|} \quad \text { and } 45^{\circ}<\varsigma<90^{\circ} \quad(\text { Open Case \#3), }
$$

we find that in the open pre-existing joint case $\sigma_{3} \leq 0$ everywhere. In other words, compressive regions do not develop in the open case. The only limitations for cross joint propagation in this case are the pre-existing joint faces themselves. Therefore, both curving-parallel and curvingperpendicular cross joints that form under open conditions (i.e., $\sigma_{y y}^{\infty}>0$ ) have the capability of intersecting pre-existing joints. This result is shown in Table 3-3 and is in agreement with Dyer's conclusions. 
Table 3-3. Summary of cross joint geometries and their characteristics. In this table, $\varsigma$ represents the take off angle, $\sigma_{2}^{\infty} / \sigma_{3}^{\infty}=\eta$ is the remote principal stress ratio, $C_{0}^{*}=[(\eta-1) \sin 2 \varsigma] /[\eta(1-\cos 2 \varsigma)+(1+\cos 2 \varsigma)]$ is the critical frictional coefficient, $C_{0}$ is the coefficient of friction for the preexisting joint, $t_{0}$ is the dimension of compressive zone $R=-\left[1+\left(\cos ^{2} 2 \varsigma+s^{2} \sin ^{2} 2 \varsigma\right)^{1 / 2}\right] /\left[1-\left(\cos ^{2} 2 \varsigma+s^{2} \sin ^{2} 2 \varsigma\right)^{1 / 2}\right]$, $S=-[1+|\cos 2 \varsigma|] /[1-|\cos 2 \varsigma|]$, and $T=-[1-|\cos 2 \varsigma|] /[1+|\cos 2 \zeta|]$.

\begin{tabular}{|c|c|c|c|c|}
\hline & $\frac{\sigma_{2}^{\infty}}{\sigma_{3}^{\infty}}<R$ & $R<\frac{\sigma_{2}^{\infty}}{\sigma_{3}^{\infty}}<S$ & $S<\frac{\sigma_{2}^{\infty}}{\sigma_{3}^{\infty}}<T$ & $T<\frac{\sigma_{2}^{\alpha}}{\sigma_{3}^{\alpha}} \leq 1$ \\
\hline $0^{\circ}<\varsigma<45^{\circ}$ & \multicolumn{2}{|c|}{$\begin{array}{c}\text { CLOSED CASE \#1 } \\
\sigma_{y}^{\infty}>0, \sigma_{z}^{\infty}>0 \\
\text { quasi-curving } \\
\text { parallel } \\
\mathbf{t}_{\mathbf{0}} \geq \mathbf{0}\end{array}$} & $\begin{array}{c}\text { OPEN CASE \#1 } \\
\sigma_{y}^{\infty}<0, \sigma_{z}^{\infty}>0 \\
\text { curving-parallel }\end{array}$ & $\begin{array}{c}\text { OPEN CASE \#2 } \\
\sigma_{y}^{\infty}<0, \sigma_{z}^{\infty}<0 \\
\text { curving- } \\
\text { perpendicular }\end{array}$ \\
\hline \multirow{4}{*}{$45^{\circ}<\varsigma<90^{\circ}$} & \multicolumn{2}{|c|}{$\begin{array}{c}\text { CLOSED CASE \#2 } \\
\sigma_{v}^{\infty}>0, \sigma_{z}^{\infty}>0\end{array}$} & $\begin{array}{c}\text { CLOSED CASE \# } \\
\sigma_{v}^{\infty}>0, \sigma_{2}^{\infty}<0\end{array}$ & \multirow{4}{*}{$\begin{array}{c}\text { OPEN CASE \#3 } \\
\sigma_{y}^{\infty}<0, \sigma_{z}^{\infty}<0 \\
\\
\text { curving- } \\
\text { perpendicular }\end{array}$} \\
\hline & \multicolumn{2}{|c|}{$\begin{array}{c}\text { Closed Case \#2a } \\
C_{0} \leq C_{0}^{*} \\
\text { unlocked pre- } \\
\text { existing joint } \\
\text { quasi-curving- } \\
\text { perpendicular }\end{array}$} & \multirow[t]{2}{*}{$\begin{array}{c}\text { Closed Case \#3a } \\
C_{0} \leq C_{0}^{*} \\
\text { unlocked } \\
\text { pre-existing joint }\end{array}$} & \\
\hline & $\begin{array}{l}\text { Closed } \\
\text { Case } \\
\# 2 a I \\
\mathbf{t}_{0} \geq \mathbf{0}\end{array}$ & $\begin{array}{c}\text { Closed } \\
\text { Case } \\
\# 2 \text { alI } \\
\mathbf{t}_{\mathbf{0}}=\mathbf{0}\end{array}$ & & \\
\hline & \multicolumn{2}{|c|}{$\begin{array}{c}\text { Closed Case \#2b } \\
C_{0}>C_{0}^{*} \text { or } s=1 \\
\text { locked pre-existing } \\
\text { joint } \\
\text { non-curving } \\
\mathbf{t}_{\mathbf{0}}=\mathbf{0}\end{array}$} & $\begin{array}{c}\text { Closed Case\#3b } \\
C_{0}>C_{0}^{*} \text { or } s=1 \\
\text { locked } \\
\text { pre-existing joint } \\
\text { non-curving } \\
\mathbf{t}_{\mathbf{0}}=\mathbf{0}\end{array}$ & \\
\hline
\end{tabular}




\section{CLOSED PRE-EXISTING JOINT CASE}

According to equations (3-29), (3-30), (3-31), (3-1), (3-2) and (3-3), the derived local principal stresses for the closed pre-existing case are

$$
\sigma_{2,3}=\frac{\sigma_{2}^{\infty}+\sigma_{3}^{\infty}}{2} \pm \frac{\sigma_{2}^{\infty}-\sigma_{3}^{\infty}}{2}\left\{\cos ^{2} 2 \varsigma+\left[s+(1-s) \frac{y}{\left(y^{2}+c^{2}\right)^{1 / 2}}\right]^{2} \sin ^{2} 2 \varsigma\right\}^{1 / 2} .
$$

From Table 3-1, we know the conditions for the closed pre-existing case are

$-\frac{1+|\cos 2 \varsigma|}{1-|\cos 2 \varsigma|}<\eta<-\frac{1-|\cos 2 \varsigma|}{1+|\cos 2 \varsigma|} \quad$ and $45^{\circ}<\varsigma<90^{\circ}$ (Closed Case \#3),

or

$\eta<-\frac{1+|\cos 2 \varsigma|}{1-|\cos 2 \varsigma|} \quad$ and $\quad 0^{\circ}<\varsigma<90^{\circ} \quad($ Closed Case \#1 and \#2).

First, let us consider $\sigma_{2}$. From (3-45), we have

$$
\sigma_{2} \geq \frac{\sigma_{2}^{\infty}+\sigma_{3}^{\infty}}{2}+\frac{\sigma_{2}^{\infty}-\sigma_{3}^{\infty}}{2}|\cos 2 \varsigma|
$$

Under the conditions of (3-46), (3-47) and (3-48), we can derive that $\sigma_{2}>0$. Therefore, $\sigma_{2}$ is compressive everywhere in the closed pre-existing joint cases.

Second, let us consider $\sigma_{3}$ in several steps. 
(i) For Closed Case \#3 in Table 3-1, we know that

$$
-\frac{1+|\cos 2 \varsigma|}{1-|\cos 2 \varsigma|}<\eta<-\frac{1-|\cos 2 \varsigma|}{1+|\cos 2 \varsigma|} \quad \text { and } 45^{\circ}<\varsigma<90^{\circ} \text {. }
$$

From (3-45), we have

$$
\sigma_{3}=\frac{\sigma_{2}^{\infty}+\sigma_{3}^{\infty}}{2}-\frac{\sigma_{2}^{\infty}-\sigma_{3}^{\infty}}{2}\left\{\cos ^{2} 2 \varsigma+\left[s+(1-s) \frac{y}{\left(y^{2}+c^{2}\right)^{1 / 2}}\right]^{2} \sin ^{2} 2 \varsigma\right\}^{1 / 2} .
$$

From this expression, the following inequality is obtained

$$
\sigma_{3} \leq \frac{\sigma_{2}^{\infty}+\sigma_{3}^{\infty}}{2}-\frac{\sigma_{2}^{\infty}-\sigma_{3}^{\infty}}{2}|\cos 2 \varsigma|
$$

which may be converted to

$$
\sigma_{3} \leq \frac{1}{2}\left[\sigma_{2}^{\infty}(1-|\cos 2 \varsigma|)+\sigma_{3}^{\infty}(1+|\cos 2 \varsigma|)\right]
$$

From the conditions outlined in equation (3-49), we have

$$
\sigma_{2}^{\infty}(1-|\cos 2 \varsigma|)+\sigma_{3}^{\infty}(1+|\cos 2 \varsigma|)<0 .
$$

Therefore, $\sigma_{3}^{\infty}<0$, i.e., there is no compressive zone for the Closed Case \#3 (Table 3-3).

(ii) For Closed Cases \#1 and \#2, we have 


$$
\eta<-\frac{1+|\cos 2 \varsigma|}{1-|\cos 2 \varsigma|} \text { and } 0^{\circ}<\varsigma<90^{\circ}
$$

In terms of $\eta$ and $t$, and according to equation (3-50), $\sigma_{3}$ can be expressed as

$$
\sigma_{3}=\frac{\sigma_{3}^{\infty}(\eta+1)}{2}-\frac{\sigma_{3}^{\infty}(\eta-1)}{2}\left\{\cos ^{2} 2 \varsigma+\left[s+(1-s) \frac{t}{\left(t^{2}+1\right)^{1 / 2}}\right]^{2} \sin ^{2} 2 \varsigma\right\}^{1 / 2} .
$$

In order to determine the sign of $\sigma_{3}$, we need to differentiate equation (355 ) with respect to the normalized distance from the pre-existing joint $(t)$. The differentiation results in

$$
\frac{\partial \sigma_{3}}{\partial t}=-\frac{\sigma_{3}^{\infty}(\eta-1) \sin ^{2} 2 \varsigma\left[s+(1-s) \frac{t}{\left(t^{2}+1\right)^{1 / 2}}\right](1-s) \frac{1}{\left(t^{2}+1\right)^{3 / 2}}}{2\left\{\cos ^{2} 2 \varsigma+\left[s+(1-s) \frac{t}{\left(t^{2}+1\right)^{1 / 2}}\right]^{2} \sin ^{2} 2 \varsigma\right\}^{1 / 2}} .
$$

From (3-56), we can see that $\frac{\partial \sigma_{3}}{\partial t} \leq 0$, for $0 \leq s \leq 1$ and $\eta<0$, which is clear from equation (3-54). It means that $\sigma_{3}$ decreases in value with increasing normalized distance from the pre-existing joint.

Now let us evaluate the value of $\sigma_{3}$ at $t \rightarrow \infty$, and $t=0$. From (3-55), we have

$$
\sigma_{3}(t \rightarrow \infty)=\frac{\sigma_{3}^{\infty}(\eta+1)}{2}-\frac{\sigma_{3}^{\infty}(\eta-1)}{2}=\sigma_{3}^{\infty}<0
$$


At $t=0$, from (3-57), we have

$$
\sigma_{3}(t=0)=\frac{\sigma_{3}^{\infty}(\eta+1)}{2}-\frac{\sigma_{3}^{\infty}(\eta-1)}{2}\left(\cos ^{2} 2 \varsigma+s^{2} \sin ^{2} 2 \varsigma\right)
$$

Suppose $\sigma_{3}(t=0)>0$, we have

$$
\eta<-\frac{1+\left(\cos ^{2} 2 \varsigma+s^{2} \sin 2 \varsigma\right)^{1 / 2}}{1-\left(\cos ^{2} 2 \varsigma+s^{2} \sin 2 \varsigma\right)^{1 / 2}}
$$

Therefore, if equation (3-59) can be satisfied, there must be a $t=t_{0}$ between 0 and $\infty$ where $\sigma_{3}=0$, such that for $t<t_{0}, \sigma_{3}>0$, and when $t>t_{0}, \sigma_{3}<0$. In other words, $t=t_{0}$ defines a line, which is parallel to the pre-existing joint and represents the transition from compressive least principal stress near the pre-existing joint to a field marked by tensile least principal stress. The magnitude of $t_{0}$ gives the dimension of the compressive zone. By assuming $\sigma_{3}=0$ in $(3-55)$, we can calculate $t_{0}$ as

$$
t_{0}=\frac{\left|\left[\left(((\eta+1) /(\eta-1))^{2}-\cos ^{2} 2 \varsigma\right) / \sin ^{2} 2 \varsigma\right]^{1 / 2}-s\right|}{\left\{(1-s)^{2}-\left(\left[\left(((\eta+1) /(\eta-1))^{2}-\cos ^{2} 2 \varsigma\right) / \sin ^{2} 2 \varsigma\right]^{1 / 2}-s\right)^{2}\right\}^{1 / 2}} .
$$

On the other hand, if equation (3-59) cannot be satisfied, we have $\sigma_{3}(t=0) \leq 0$, which means that a compressive zone does not exist.

For Closed Case \#1, by substituting equations (3-1) and (3-3) as well as $s=C_{0} \sigma_{y y}^{\infty} / \sigma_{y z}^{\infty}$ (because $C_{0}^{*} \geq 1$ for Closed Case \#1, refer to equation (3-37), 
into equation (3-59), we determine the range of the remote principal stress ratio $(\eta)$

$$
\eta<-\frac{1+\cos 2 \varsigma}{1-\cos 2 \varsigma}
$$

Equation (3-61) corresponds to the condition for Closed Case \#1.

Therefore, in Closed Case \#1, there is a compressive zone with dimension of $t_{0}$ expressed as in equation (3-60). For Closed Case \#2, it is clear that

$$
-\frac{1+\left(\cos ^{2} 2 \varsigma+s^{2} \sin 2 \varsigma\right)^{1 / 2}}{1-\left(\cos ^{2} 2 \varsigma+s^{2} \sin 2 \varsigma\right)^{1 / 2}} \leq-\frac{1+|\cos 2 \varsigma|}{1-|\cos 2 \varsigma|} .
$$

Therefore, we can subdivide Closed Case \#2a (see Table 3-2) more specifically according to the value of the remote principal stress ratio. If $\eta$ is less than or equal to the left side of equation (3-62), there is a compressive zone with a dimension of $t_{0}$ which is described by equation (360) (Closed Case \#2aI in Table 3-3). However, if $\eta$ is greater than the left side of equation (3-62) and less than the right side of equation (3-62), there is no compressive zone (Closed Case \#2aII) in Table 3-3). Most importantly, when $s=1$, i.e., the pre-existing joint is locked, the dimension of the compressive zone is zero.

Figure 3-15a shows the plots of the dimension of the compressive zone $\left(t_{0}\right)$ versus the remote principal stress ratio $(\eta)$ by assuming a take-off angle of $30^{\circ}$, and $C_{0}=0$ and 0.6 , respectively. Figure $3-15 \mathrm{~b}$ shows the compressive zone dimension $\left(t_{0}\right)$ as a function of take-off angle $(\varsigma)$, assuming the remote principal stress ratio $(\eta)$ is -5 , and a frictional 

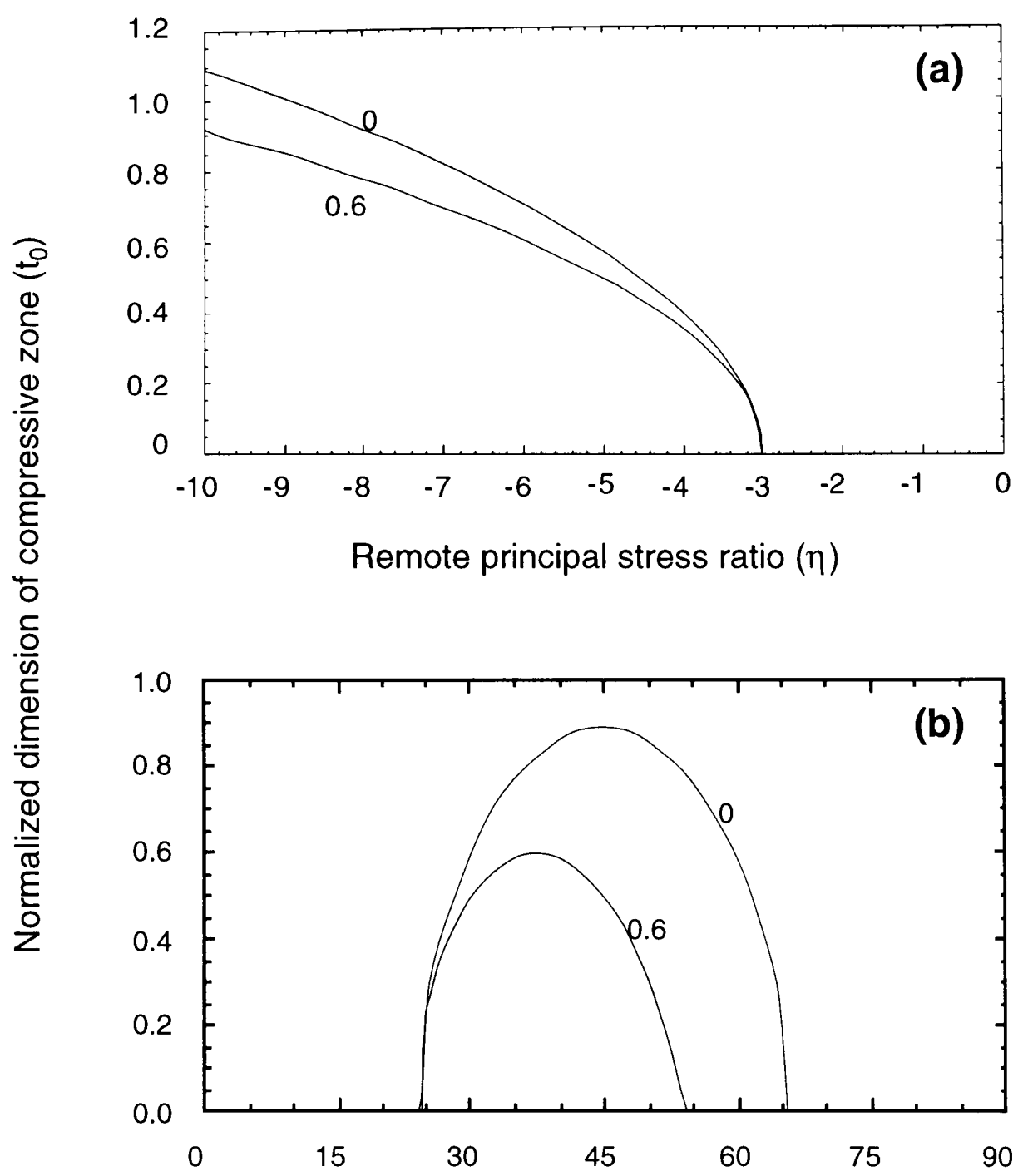

Take off angle $(\varsigma)$

Fig. 3-15. (a) Dimension of compressive zone $\left(t_{0}\right)$ as a function of remote principal stress ratio $(\eta)$. These plots represent examples of the Closed Case \#1 in Table 3-2, in which $\varsigma=30^{\circ}, C_{0}=0$ and 0.6, respectively. (b) Dimension of compressive zone variation as a function of take off angle $(\varsigma)$ under the condition for a closed preexisting joint. The number next to each curve, in both (a) and (b), indicates the coefficient of friction along the pre-existing joint. 
coefficient of 0 and 0.6 , respectively. The plots predict large compressive zones for more negative stress ratios (Fig. 3-15a), while a greater value of $\sigma_{3}^{\infty}\left(\sigma_{3}^{\infty} \leq 0\right)$ corresponds to a greater value of $\sigma_{2}^{\infty}\left(\sigma_{2}^{\infty} \geq 0\right)$. In other words, the greater the maximum horizontal stress component, the greater the compressive zone.

The presence or absence of a compressive zone depends to a large extent on the take-off angle; for cross joints that take-off nearly parallel or perpendicular to the pre-existing joint, compressive zones do not exist because either $\sigma_{y y}$ or $\sigma_{z z}$ is tensile (i.e., negative). Consequently, with respect to the take-off angle, the maximum compressive zone dimension is found at an intermediate value of the take-off angle (Fig. 3-15b).

The detailed categorization of cross joint geometries according to such characteristics as remote principal stress ratio, take-off angle, frictional coefficient, and compressive zone is summarized in Table 3-3. For convenience in using Table 3-3, the results are also shown diagrammatically in Fig. 3-16. In Fig. 3-16a, a perfectly lubricated preexisting joint is assumed (i.e., $C_{0}$ is zero). In Fig. 3-16b, a frictional coefficient of 0.6 was used in the plot.

\section{INFLUENCES OF PORE PRESSURE ON CROSS JOINT GROWTH}

Until now the effect of pore pressure has not been considered in the treatment of cross joint growth. In the following section, I will discuss the role of pore pressure in controlling the geometry of cross joints. 

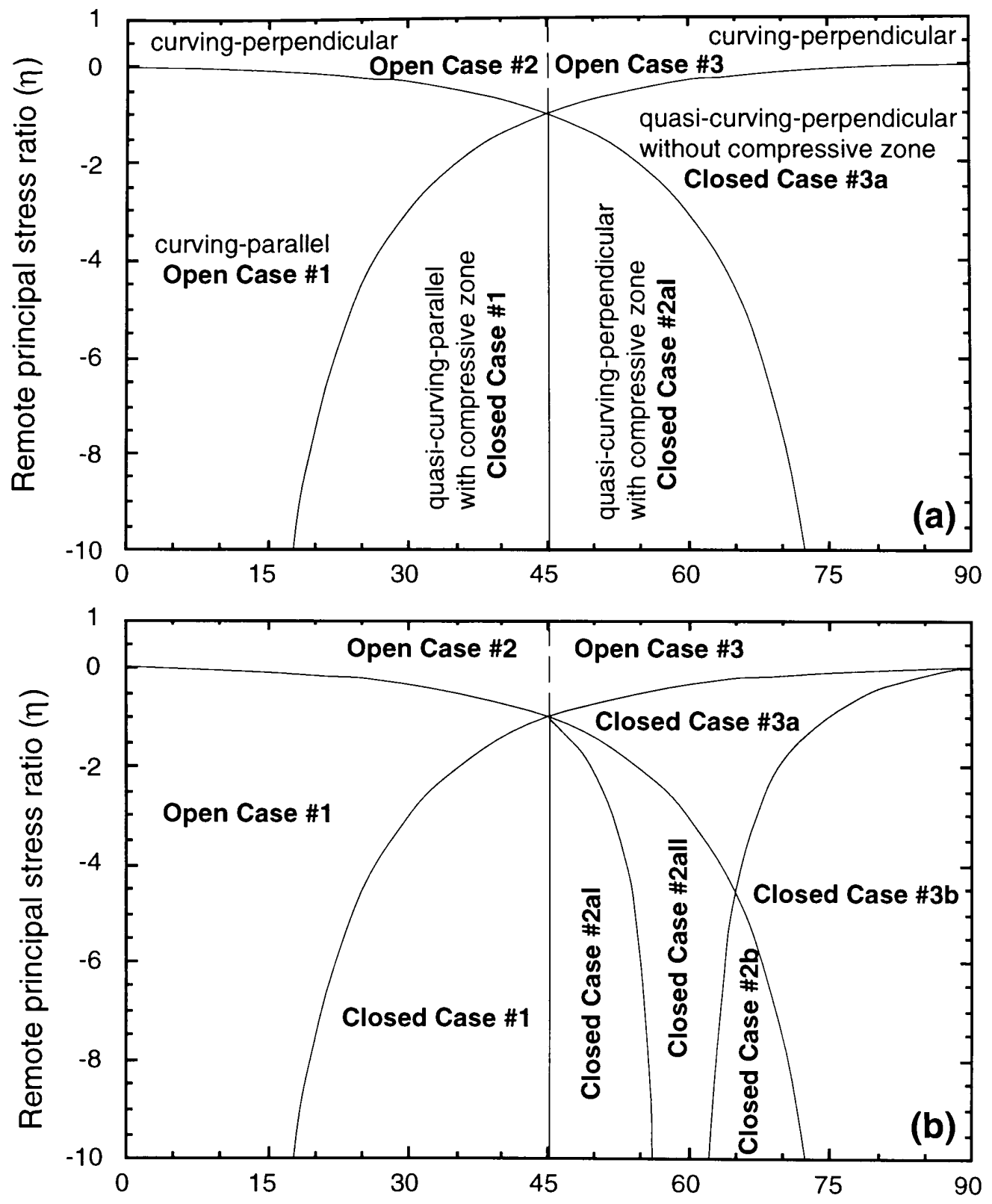

Take off angle $(\varsigma)$

Fig. 3-16. Cross joint geometries and their characteristics as a function of takeoff angle $(\varsigma)$ and remote principal stress ratio ( $\eta$ ). (a) Completely lubricated pre-existing joint case (i.e., $C_{0}=0$ ). (b) The case when the coefficient of friction along the pre-existing joint $\left(C_{0}\right)$ is 0.6 . 
According to fracture mechanics theory (Lawn and Wilshaw, 1975; Cherepanov, 1979; Broek, 1986), mode-I fractures form in response to a tensile stress in the direction normal to the fracture face. That means $\sigma_{3}<0$ in terms of our coordinate system and the conventions we used in the previous sections. Although local tensile stresses may exist in some special structural positions such as above a neutral surface in an anticline, pure tensile stresses are rarely found on regional scales, especially at depths greater than several hundred meters. One explanation for the pervasive distribution of regional joint sets found both in outcrops and in the subsurface is the role played by pore pressure.

To consider the role of pore pressure, I first introduce the concepts of effective stresses and effective remote principal stress ratio. Then, by incorporating the concepts of effective stresses and effective remote principal stress ratio into the results from the previous sections, I discuss influences of pore pressure on cross joint geometry.

\section{EFFECTIVE STRESS AND EFFECTIVE REMOTE PRINCIPAL STRESS RATIO}

The general expression for the effective stress tensor is defined as

$$
\bar{\sigma}_{i j}=\sigma_{i j}-\alpha P_{p} \delta_{i j},
$$

where $\sigma_{i j}$ represents stress in the $j$ direction acting on the plane normal to the $i$ direction, $P_{p}$ is pore pressure, $\delta_{i j}$ is Kroenecker's delta, and $\alpha$ is the 
Biot pore-pressure coefficient. The Biot pore-pressure coefficient, in turn can be expressed as

$$
\alpha=1-K / K_{s},
$$

where $K$ is the bulk modulus of the saturated rock, and $K_{s}$ is the intrinsic modulus of the rock material (Nur and Byerlee, 1971). The commonly used effective stress law is a special form of equation (3-63) which assumes $\alpha=1$ (Jaeger and Cook, 1979, Engelder, 1993), and is expressed as

$$
\bar{\sigma}_{i j}=\sigma_{i j}-P_{p} \delta_{i j} .
$$

In the following section, I will use the effective stress law described by equation (3-65) to study the role of pore pressure in controlling cross joint growth.

If the pore pressure is uniformly distributed in the elastic domain (Fig. 3-3), the analysis in the former sections can be recast in terms of effective stresses defined by equation (3-65). All the analyses will be valid if we substitute effective stresses into the former equations.

By incorporating pore pressure, we can change the remote principal stress ratio into the following form, which is

$$
\eta^{*}=\frac{\sigma_{2}^{\infty}-P_{p}}{\sigma_{3}^{\infty}-P_{p}}
$$


where $\eta^{*}$ is refered to as the effective principal stress ratio. Rewriting equation (3-66) yields

$$
\eta^{*}=\frac{\sigma_{2}^{\infty} / \sigma_{3}^{\infty}-P_{p} / \sigma_{3}^{\infty}}{1-P_{p} / \sigma_{3}^{\infty}} .
$$

This equation describes relationships among the remote principal stress ratio $\left(\sigma_{2}^{\infty} / \sigma_{3}^{\infty}\right.$, i.e., $\left.\eta\right)$, effective remote principal stress ratio $\left(\eta^{*}\right)$ and pore pressure to least principal stress ratio $\left(P_{p} / \sigma_{3}^{\infty}\right)$. A plot of $\eta^{*}$ versus $\sigma_{2}^{\infty} / \sigma_{3}^{\infty}$ at $P_{p} / \sigma_{3}^{\infty}=1.1,1.5$ and 2.0, respectively, is shown in Fig. 3-17a. In Fig. 3$17 \mathrm{~b}$, the relation between $\eta^{*}$ versus $P_{p} / \sigma_{3}^{\infty}$ is plotted at $\eta=1.5,2.0,3.0$ and 4.0, respectively. Figure 3-17a shows that the relation between remote principal stress ratio $\left(\sigma_{2}^{\infty} / \sigma_{3}^{\infty}\right.$, i.e., $\left.\eta\right)$ and effective principal stress ratio $\left(\eta^{*}\right)$ depends upon the pore pressure to least horizontal stress ratio $\left(P_{p} / \sigma_{3}^{\infty}\right)$; at the same pore pressure to least horizontal stress ratio $\left(P_{p} / \sigma_{3}^{\infty}\right)$, the effective remote principal stress ratio $\left(\eta^{*}\right)$ decreases with increasing remote principal stress ratio $(\eta)$. Figure $3-17 \mathrm{~b}$ shows that effective principal stress ratio $\left(\eta^{*}\right)$ increases with increasing pore pressure to least horizontal stress ratio $\left(P_{p} / \sigma_{3}^{\infty}\right)$ for the same remote principal stress ratio $(\eta)$. 

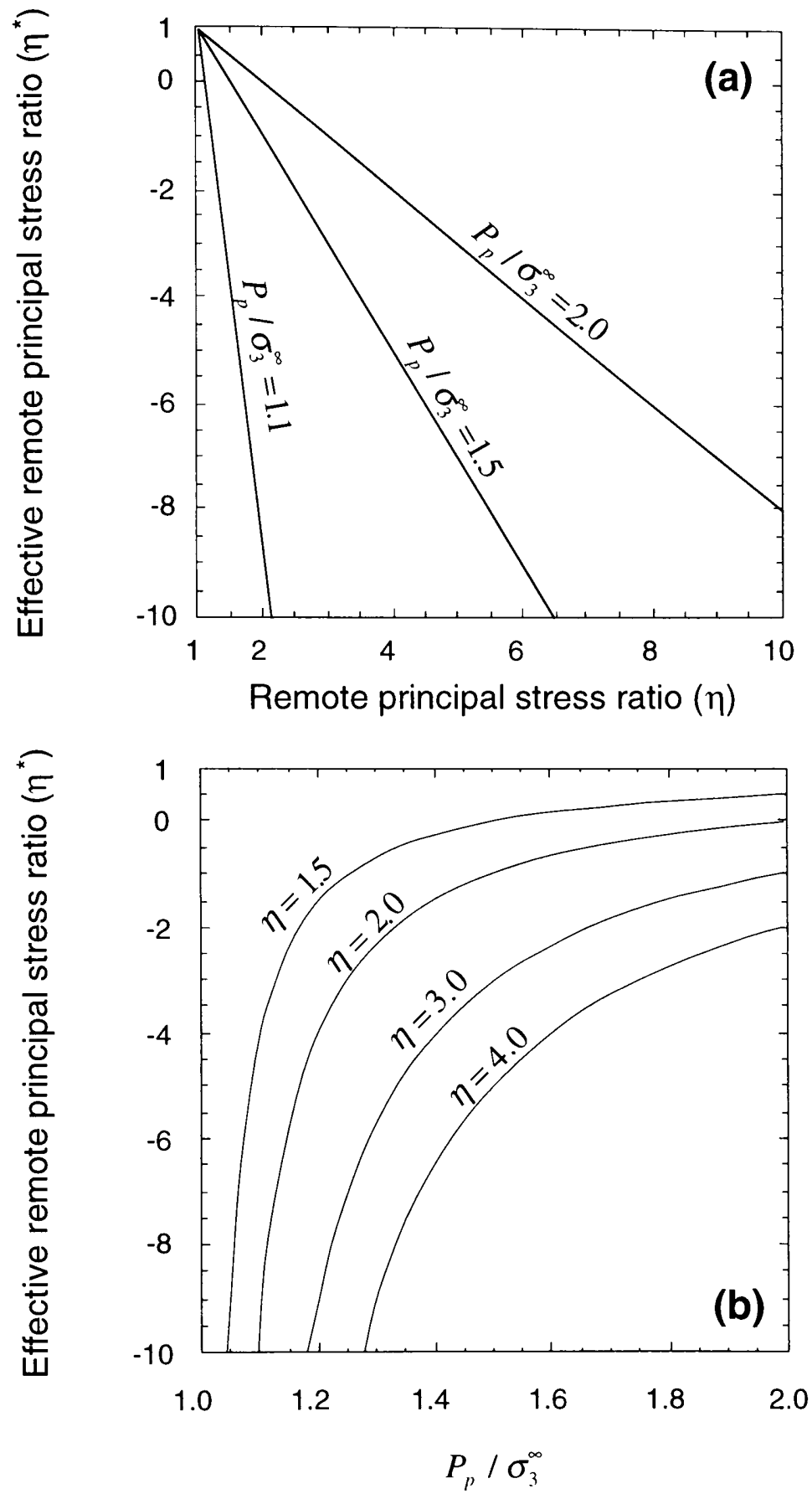

Fig. 3-17. (a) Relations between the effective remote principal stress ratio $\left(\eta^{*}\right)$ and the remote principal stress ratio $(\eta)$. (b) Relations between the effective remote principal stress ratio $\left(\eta^{*}\right)$ and the pore pressure to the least principal stress ratio $\left(P_{p} / \sigma_{3}^{\infty}\right)$. 


\section{CROSS JOINT GEOMETRY AND PORE PRESSURE}

In order to study the role of pore pressure in controlling the cross joint geometry, let us discuss two different cases.

First, let us consider take-off angles less than $45^{\circ}$. As described in Table 3-3, when the take-off angle $(\varsigma)$ is less than $45^{\circ}$, cross joint geometry will vary from quasi-curving-parallel with a compressive zone to curvingparallel to curving-perpendicular with increasing remote principal stress ratio $(\eta)$, and these changes are independent of the coefficient of friction along the pre-existing joint. As an example, I will investigate cross joint paths with a take-off angle of $30^{\circ}$, which is Dyer's special case. As stated previously, for Dyer's case curving-parallel geometries occur when the remote stress ratio is between -3 and $-1 / 3$, whereas curving-perpendicular geometries occur when the remote principal stress ratio is between $-1 / 3$ and 1. These statements hold true for rocks subjected to internal pore pressure if we substitute the remote principal stress ratio with the effective remote principal stress ratio. In equation (3-67), if we suppose $\eta^{*}=-3,-1 / 3$ and 1 , respectively, and both of the principal stresses are compressive (i.e., positive), we can draw the ranges for different cross joint geometries corresponding to Closed Case \#1, and Open Cases \#1 and \#2 in Table 3-3, which are shown in Fig. 3-18.

From Fig. 3-18, we can see that cross joint geometry will change

with increasing pore pressure for a given remote principal stress ratio $\left(\sigma_{2}^{\infty} / \sigma_{3}^{\infty}\right)$. When the pore pressure is low $\left(P_{p} / \sigma_{3}^{\infty}<1\right)$, jointing cannot occur because both of the principal effective stresses are positive, i.e., the whole region is in a compressive condition. However, when pore pressure exceeds 


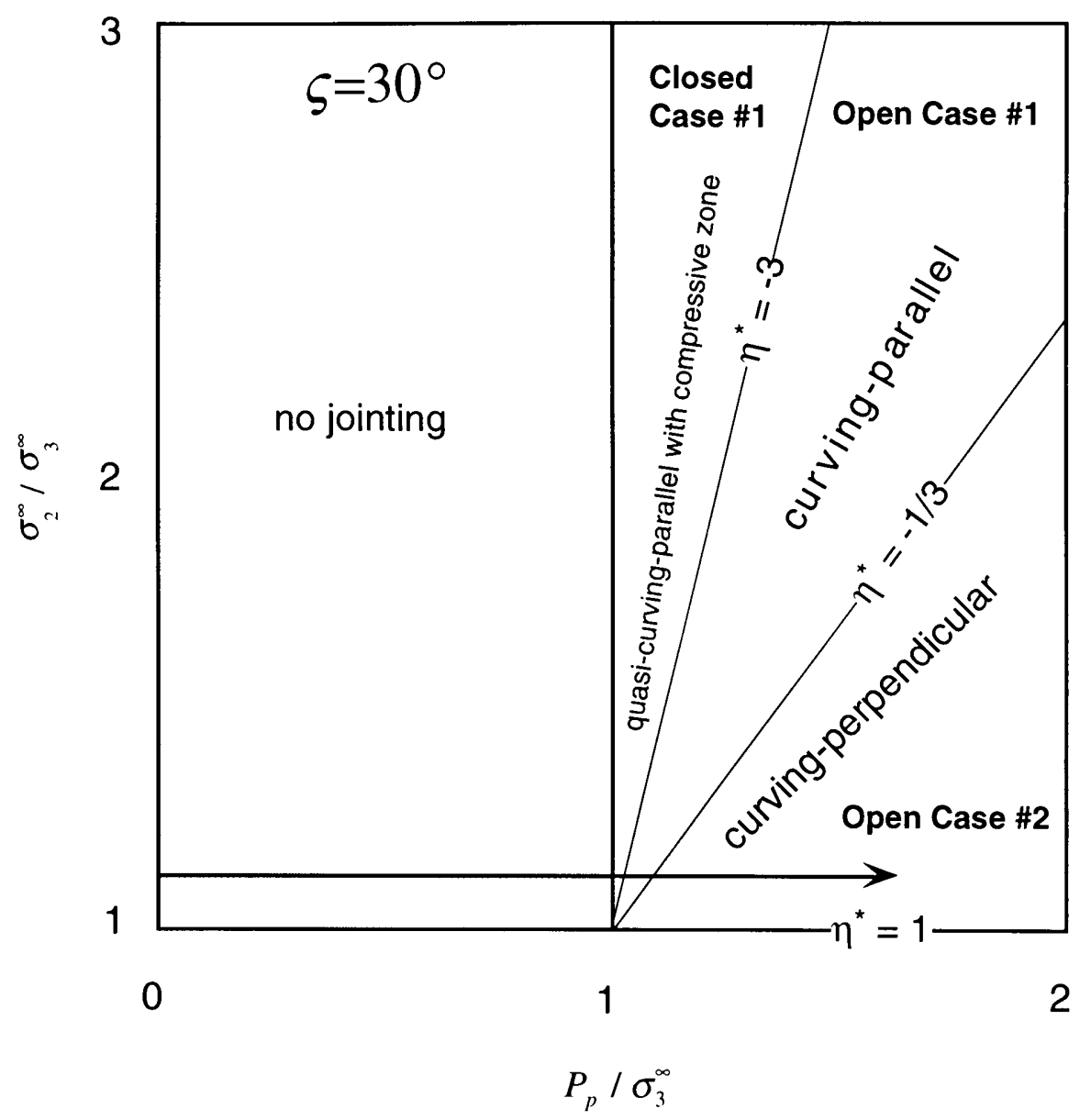

Fig. 3-18. Cross joint geometry variation as a function of pore pressure.

The arrow shows that cross joint geometry will change from quasicurving-parallel with compressive zone (Closed Case \#1) to curvingparallel (Open Case \#1) to curving-perpendicular (Open Case \#2) as pore pressure increases. This figure shows the case in which the takeoff angle $\varsigma=30^{\circ}$. 


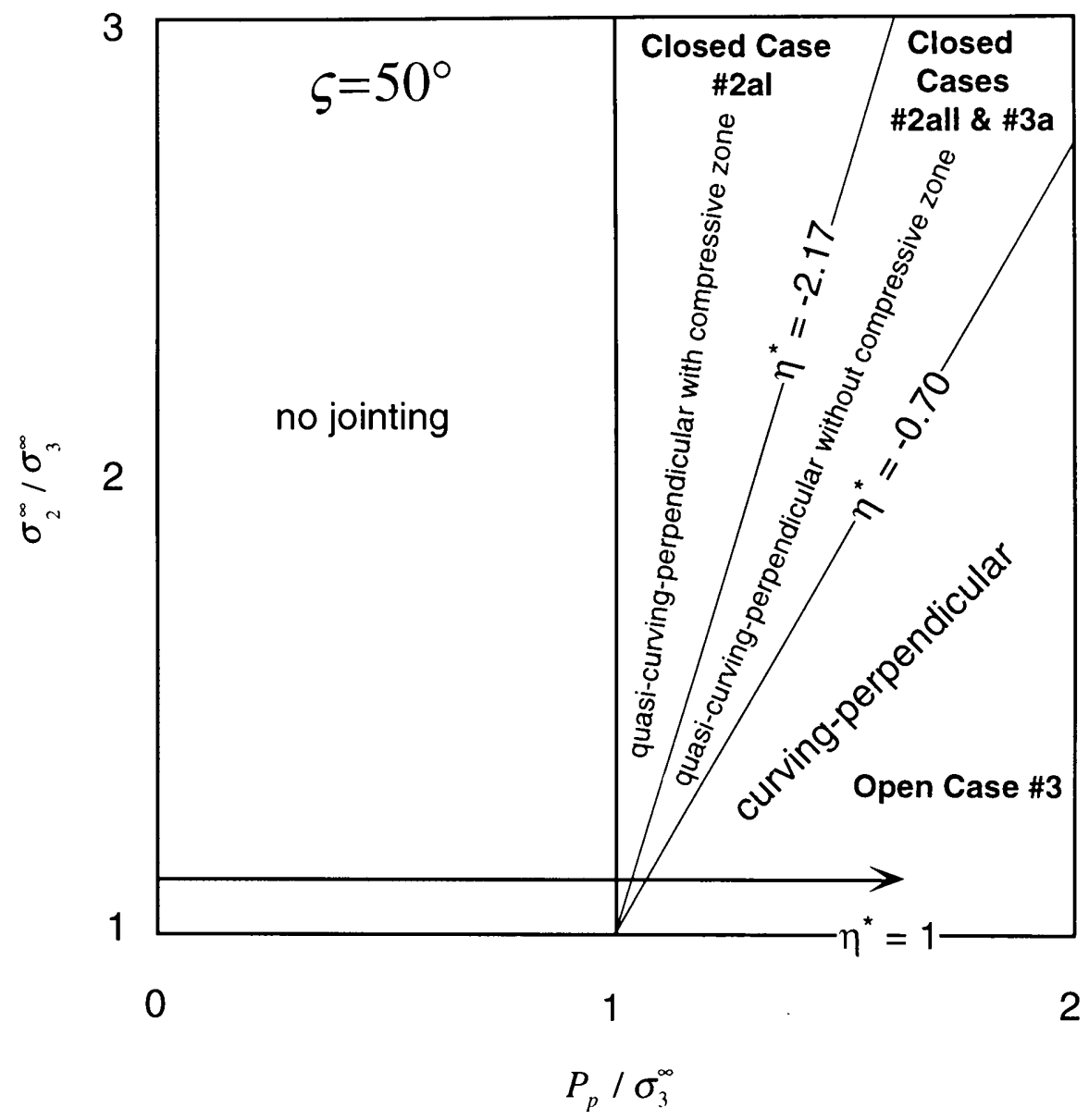

Fig. 3-19. Cross joint geometry variation as a function of pore pressure.

The arrow shows that cross joint geometry will change from quasicurving-perpendicular with compressive zone (Closed Case \#2aI) to quasi-curving-perpendicular without compressive zone (Closed Cases \#2aII and \#3a) to curving-perpendicular (Open Case \#3) as pore pressure increases. This figure shows the case in which the takeoff angle $\varsigma=50^{\circ}$. 
the least principal stress (i.e., $P_{p} / \sigma_{3}^{\infty}>1$ ), cross joint geometry will change from quasi-curving-parallel (Closed Case \#1) to curving-parallel (Open Case \#2) to curving-perpendicular (Open Case \#3) with increasing pore pressure. The arrow in Fig. 3-18 shows the change of cross joint geometry with increasing pore pressure.

Second, we now consider the case of take-off angles greater than $45^{\circ}$ and less than $90^{\circ}$, corresponding to Closed Cases \#2 and \#3, and Open Case \#3 in Table 3-3. Situations where take-off angles are greater than $45^{\circ}$ are more complex than those with take-off angle less than $45^{\circ}$, because when $45^{\circ}<\varsigma<90^{\circ}$ cross joint geometries not only depend upon take-off angle $(\varsigma)$ and remote principal stress ratio ( $\eta$ ) but also upon the coefficient of friction $\left(C_{0}\right)$ along the pre-existing joint. Due to this increased complexity, the relationship between cross joint geometry and pore pressure are evaluated separately for take-off angles of $50^{\circ}, 60^{\circ}, 70^{\circ}$ and $80^{\circ}$.

(1) Take-off angle $(\varsigma)$ is $50^{\circ}$. For $\varsigma=50^{\circ}$ cross joint geometry will change from quasi-curving-perpendicular with a compressive zone (Closed Case \#2aI) to quasi-curving-perpendicular without a compressive zone (Closed Cases \#2aII and \#3a) to curving-perpendicular (Open Case \#3) (Fig. 3-16). The boundary between Open Case \#3 and Closed Case \#3a in terms of effective remote principal stress ratio is defined by (refer to Table 3-3)

$$
\eta^{*}=-\frac{1-|\cos 2 \varsigma|}{1+|\cos 2 \varsigma|},
$$

and the boundary between Closed Case \#2aI and Closed Case \#2aII is defined by 


$$
\eta^{*}=-\frac{1+\left(\cos ^{2} 2 \varsigma+s^{2} \sin ^{2} 2 \varsigma\right)^{1 / 2}}{1-\left(\cos ^{2} 2 \varsigma+s^{2} \sin ^{2} 2 \varsigma\right)^{1 / 2}} .
$$

Because both Closed Case \#3a and Closed Case \#2aII are characterized by quasi-curving-perpendicular geometry without a compressive zone, it is not necessary to define the boundary between them. By substituting $\varsigma=50^{\circ}$ into equations (3-68) and (3-69), we have $\eta^{*}=-0.70$ and -2.17 , respectively. By setting the effective remote principal stress ratio to be -0.70 and -2.17 , respectively, in equation (3-67), we can plot $\sigma_{2}^{\infty} / \sigma_{3}^{\infty}$ versus $P_{p} / \sigma_{3}^{\infty}$, which is shown in Fig. 3-19. The arrow in Fig. 3-19 shows the predicted change in cross joint geometry as a function of increasing pore pressure while keeping $\sigma_{2}^{\infty} / \sigma_{3}^{\infty}$ as a constant.

(2) Take-off angle ( $\varsigma$ ) is $60^{\circ}$. Figure 3-16b shows that the possible cross joint geometries when $\varsigma=60^{\circ}$ are curving-perpendicular (Open Case \#3) and quasi-curving-perpendicular without a compressive zone (Closed Case \#3a and \#2aII). The boundary between them is defined by equation (3-68). Substituting $\varsigma=60^{\circ}$ into equation (3-68), we can get $\eta^{*}=-1 / 3$. Again, using $\eta^{*}=-1 / 3$ in equation (3-67), we can plot $\sigma_{2}^{\infty} / \sigma_{3}^{\infty}$ versus $P_{p} / \sigma_{3}^{\infty}$, which is shown in Fig. 3-20. Figure 3-20 shows that as pore pressure increases cross joint geometry will change from quasi-curvingperpendicular without a compressive zone to curving-perpendicular even under the condition of an unchanged remote stress field, i.e., $\quad \eta$ is a constant.

(3) Take-off angle ( $\varsigma$ ) is $70^{\circ}$. From Fig. 3-16b, we can see that the possible cross joint geometries in this case are curving-perpendicular (Open Case \#3), quasi-curving-perpendicular without compressive zone (Closed 


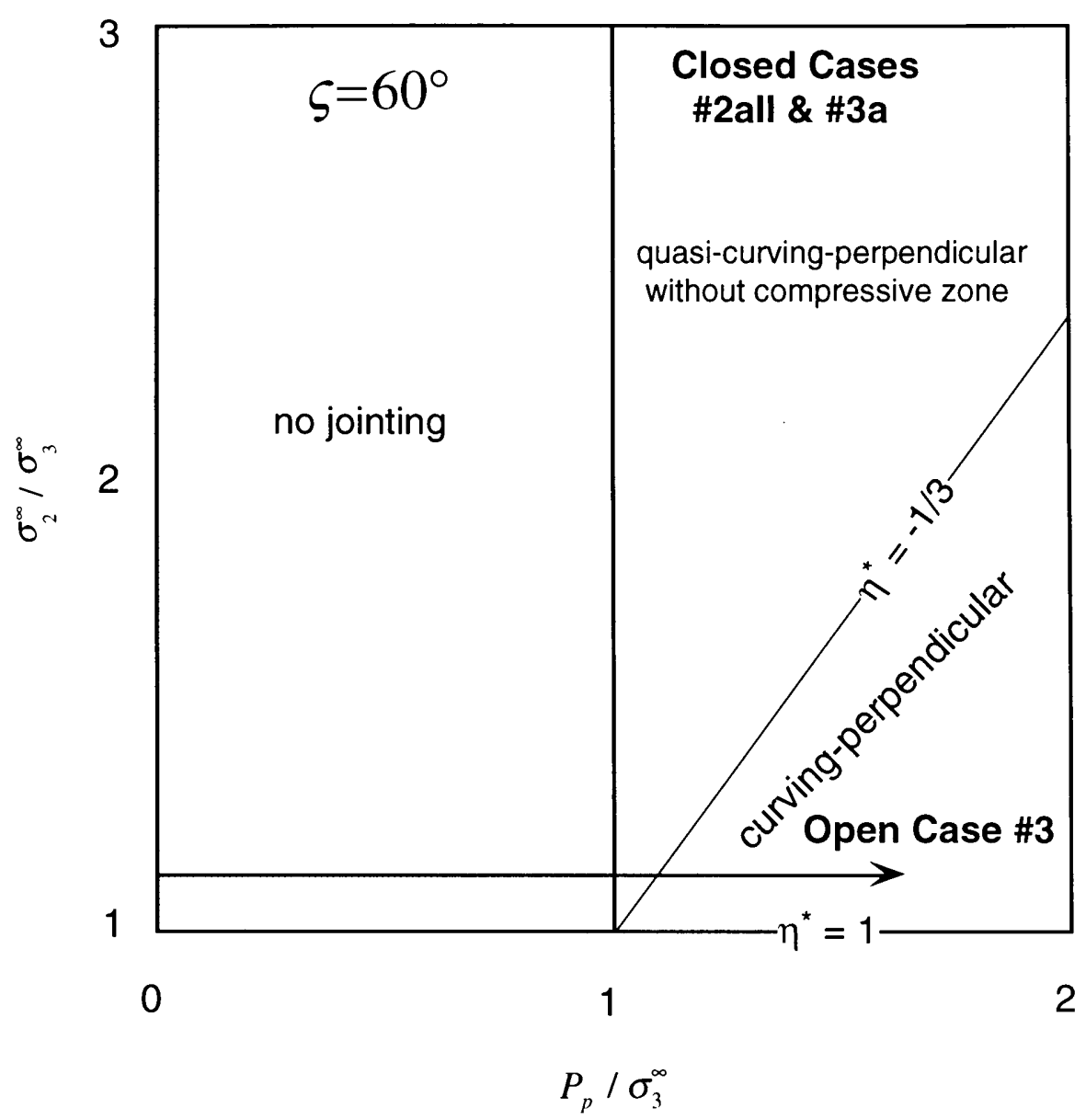

Fig. 3-20. Cross joint geometry variation as a function of pore pressure.

The arrow shows that cross joint geometry will change from quasicurving-perpendicular without compressive zone (Closed Cases \#2aII and \#3a) to curving-perpendicular (Open Case \#3) as pore pressure increases. This figure shows the case in which the takeoff angle $\varsigma=60^{\circ}$. 


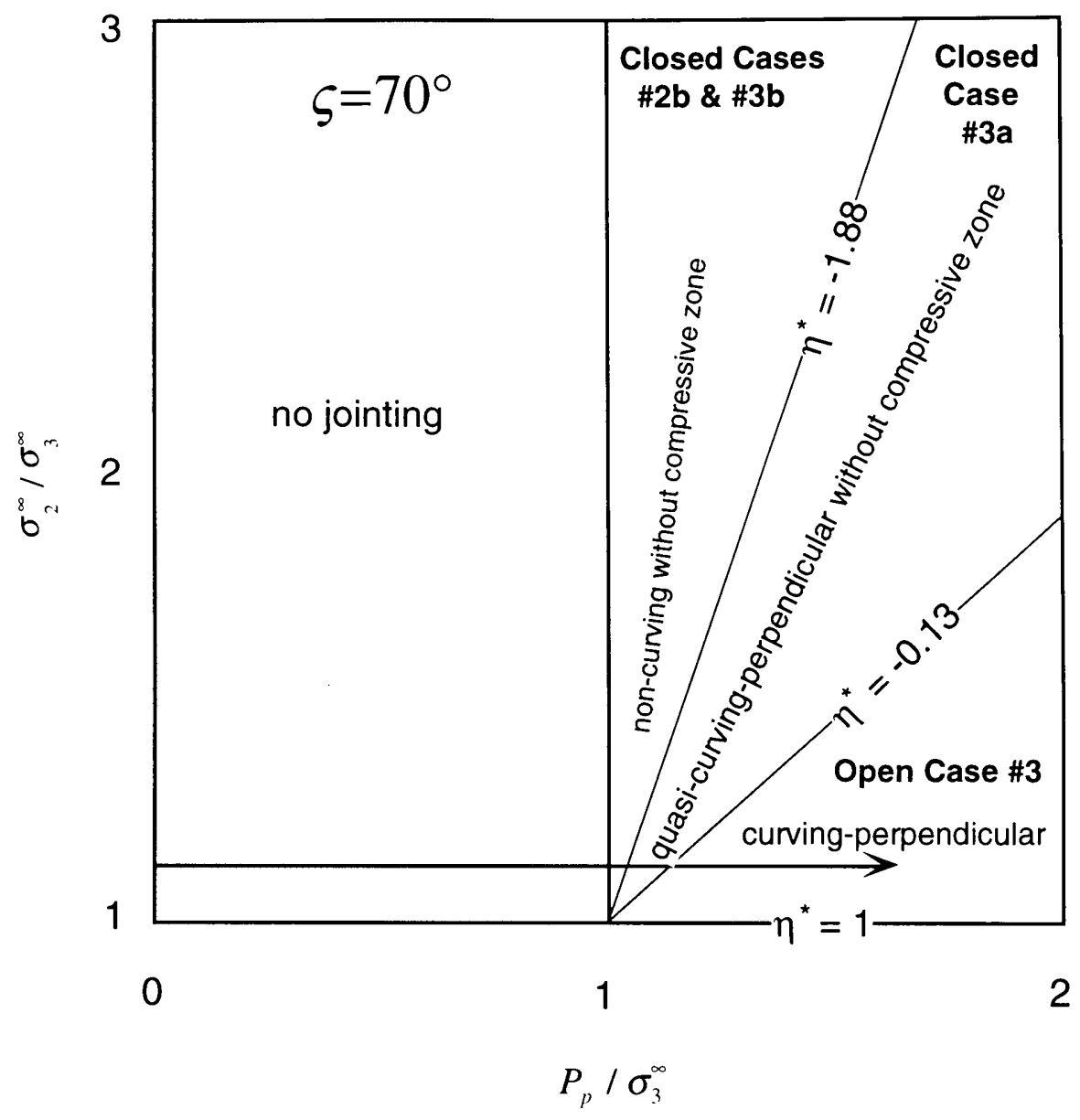

Fig. 3-21. Cross joint geometry variation as a function of pore pressure.

The arrow shows that cross joint geometry will change from noncurving without compressive zone (Closed Cases \#2b and \#3b) to quasi-curving-perpendicular without compressive zone (Closed Case \#3a) to curving-perpendicular (Open Case \#3) as pore pressure increases. This figure shows the case in which the takeoff angle $\varsigma=70^{\circ}$. 
Case \#3a) and non-curving without compressive zone (Closed Cases \#2b and \#3b). Again, the boundary between Open Case \#3 and Closed Case \#3a is defined by equation (3-68). The boundary between Closed Cases \#3a and \#3b is defined by (refer to equations (3-36), (3-37) and the corresponding discussion)

$$
C_{0}=\frac{\left(\eta^{*}-1\right) \sin 2 \varsigma}{\eta^{*}(1-\cos 2 \varsigma)+(1+\cos 2 \varsigma)}
$$

By substituting $C_{0}=0.6$ and $\varsigma=70^{\circ}$ into equation (3-70), we can get $\eta^{*}=-1.88$, and by setting $\varsigma=70^{\circ}$ in equation (3-68), we can obtain $\eta^{*}=-0.13$. Following the same procedures we can substitute $\eta^{*}=-1.88$ and -0.13 into equation (3-67), and obtain the plots of $\sigma_{2}^{\infty} / \sigma_{3}^{\infty}$ versus $P_{p} / \sigma_{3}^{\infty}$, shown in Fig. 3-21. From Fig. 3-21 we can see that cross joint geometry will change from non-curving without a compressive zone to quasi-curvingperpendicular without a compressive zone to curving-perpendicular as pore pressure increases.

(4) Take-off angle ( $\varsigma$ ) is $80^{\circ}$. The cross joint geometries in the case of $\varsigma=80^{\circ}$ are the same as for $\varsigma=70^{\circ}$ because Closed Cases \#2b and \#3b both give non-curving cross joints without compressive zones. The corresponding plots of $\sigma_{2}^{\infty} / \sigma_{3}^{\infty}$ versus $P_{p} / \sigma_{3}^{\infty}$ for $\varsigma=80^{\circ}$ are shown in Fig 322. In this case cross joint geometry will change from non-curving without a compressive zone to quasi-curving-perpendicular without a compressive zone to curving-perpendicular as pore pressure increases.

The above examples provide a general description of the relations between cross joint geometry and pore pressure in a compressive 


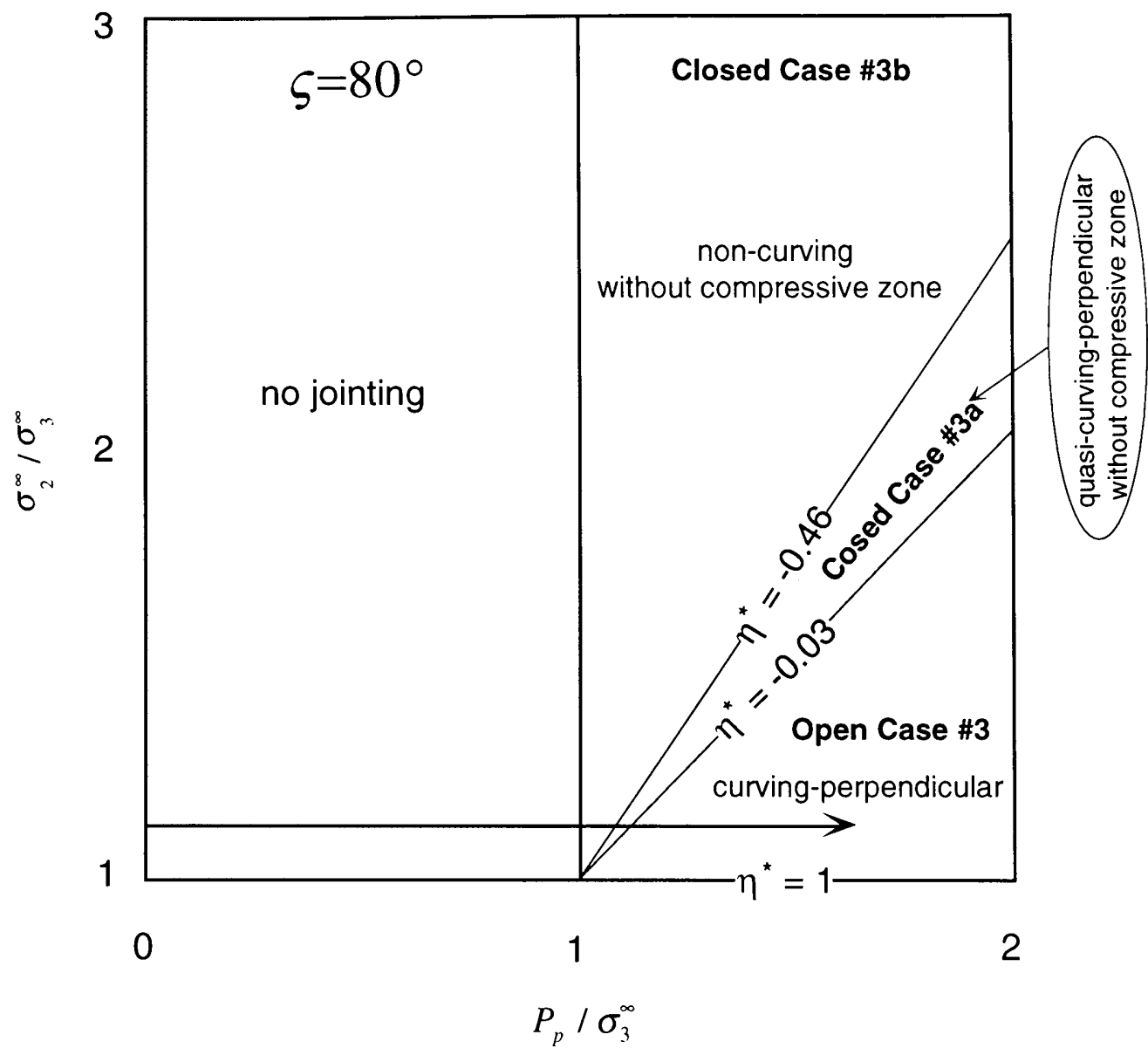

Fig. 3-22. Cross joint geometry variation as a function of pore pressure.

The arrow shows that cross joint geometry will change from noncurving without compressive zone (Closed Case \#3b) to quasicurving-perpendicular without compressive zone (Closed Case \#3a) to curving-perpendicular (Open Case \#3) as pore pressure increases. This figure shows the case in which the takeoff angle $\varsigma=80^{\circ}$. 
environment. The examples clearly demonstrate that theoretically cross joint geometry does vary as a function of pore pressure. Furthermore, cross joint geometry variations depend upon the take-off angle $(\varsigma)$, the ratio of pore pressure to least principal stress $\left(P_{p} / \sigma_{3}^{\infty}\right)$ and the remote principal stress ratio $\left(\eta\right.$, i.e., $\left.\sigma_{2}^{\infty} / \sigma_{3}^{\infty}\right)$.

\section{SUMMARY}

This chapter provides a theoretical derivation for predicting cross joint geometries based on the orientation and relative magnitudes of remote stresses, and the angular relationship with a set of pre-existing joints. The analytical solution is modeled after Dyer's (1988) treatment, which evaluates local stresses around an isolated crack in an elastic medium. The orientations and magnitudes of principal stresses adjacent to the preexisting joint will control the paths taken by cross joints and result in diagnostic intersection geometries.

The most important results of my analysis are:

(1) A general expression for local cross joint angle $(\theta)$ as a function of remote stress ratio, take-off angle, coefficient of friction, and distance from the pre-existing joint. By plotting $\theta$ as a function of distance from the pre-existing joint, one can construct cross joint trajectories for different boundary conditions. In addition to the end-member curvingparallel and curving-perpendicular geometries reported by Dyer, a number of other geometries are predicted such as quasi-curving parallel, quasicurving perpendicular, and non-curving. 
(2) A general analytical solution for the dimension of the compressive zone around the pre-existing joint. The compressive zone, which is an area adjacent to the pre-existing joint where all local principal stresses are compressive, inhibits cross joint growth. Therefore, in the absence of a compressive zone the cross joint will intersect the pre-existing joint, whereas the presence of a compressive zone will result in termination of the cross joint prior to intersection.

(3) Analytical solutions are provided that incorporate effects of pore pressure into solutions for cross joint geometry. These solutions consider cross joint growth in terms of effective stress, and can be applied to fracturing at depth, where all principle stresses are generally compressive.

(4) Based on the results of (1) - (3) a new, more detailed classification of cross joint geometries is provided. 


\section{chap?en}

\section{APPLICATION OF CROSS JOINT THEORY TO FIELD EXAMPLES}

\section{INTRODUCTION}

In the preceding chapter a series of expressions were derived in order to delineate trajectories for cross joint growth. A detailed classification of cross joints was then constructed based on cross joint geometries, remote stresses, and friction along the pre-existing joint. The purpose of this chapter is to apply results of the theoretical analysis to cross joints found in outcrop. By carefully examining geometries and orientations of cross joints in the field, one may help constrain the stress state that prevailed during the course of cross joint development, which may in turn shed light on the tectonic development of a region. 


\section{PROCEDURES FOR FIELD DATA COLLECTION AND ANALYSIS}

The procedure for analyzing cross joints in the field begins with careful descriptions of geometries and orientations. The first step is to sketch the overall joint pattern found in the outcrop, making sure to include joint trends and abutting relationships. Because cross joints curve due to mechanical interactions with other structures, it is important to document all features that may perturb or influence local structures. These include lithology, bed thickness, and height, length, and orientation of preexisting joints. Bed thickness often controls the spacing of systematic joints (e.g., Hobbs, 1967; Ladiera and Price, 1981; Huang and Angelier 1989; Gross et al. 1995), which in turn may determine whether or not the cross joint take-off angle is aligned parallel to remote intermediate principal stress; if systematic joints are closely spaced, then cross joints may not initiate as if in a homogeneous elastic medium. Furthermore, because theoretical cross joint paths are normalized to half-height of the preexisting joint $(c)$, and joint height equals bed thickness in many sedimentary rock sequences, comparisons with theoretical models require knowledge of bed thickness.

The most critical feature to measure is the cross joint trajectory, especially in areas of curvature. This can be accomplished by carefully measuring the orientation of each cross joint at various positions along its length. Alternatively, one can photograph or accurately sketch the joints in 
order to record their trajectories. The cross joint orientation at the midpoint between systematic joints should be measured; the angle between midpoint orientation and the orientation of the pre-existing joint represents the take-off angle $(\varsigma)$. If the cross joints intersect pre-existing joints, then the nature of cross joint abutments must be sketched and noted.

Termination geometries should be placed into one of the categories outlined in Table 3-3, such as curving-perpendicular, curving-parallel, non-curving, quasi-curving perpendicular, and quasi-curving parallel. In the case where cross joints terminate within the rock mass prior to reaching pre-existing joints, the distance between the cross joint tip and the pre-existing joint should be measured. This distance represents the dimension of the compressive zone $\left(\mathrm{t}_{0}\right)$ around the pre-existing joint.

With field data in hand, one can proceed with the analysis and interpretation of the cross joints. The remote least principal stress trend is perpendicular to the initial cross joint orientation as measured at the midpoint between pre-existing joints. The maximum and intermediate principal stresses lie in the plane of the cross joint. For flat-lying rocks and vertical $\sigma_{1}^{\infty}$, the intermediate remote principal stress (i.e., maximum horizontal stress) is parallel to initial cross joint trend.

The geometry of cross joint terminations can determine whether the pre-existing joint was open or closed. For pure curving-perpendicular and curving parallel geometries the pre-existing joint was open during cross joint development, otherwise it was closed (Table 3-3). A pure curvingperpendicular intersection is easily identified in the field, and indicates that local stresses parallel and normal to the pre-existing joint were both tensile 
during cross joint growth. A pure curving-parallel geometry may be more difficult to identify, and implies a compressive stress parallel to the preexisting joint.

The next step is to determine the range of the remote principal stress ratio based on take-off angle and curving geometries of cross joints outlined in Table 3-3. For the open cases, one then applies equation (3-24) along with knowledge of remote principal stress ratio and measured bed thickness to plot a series of cross joint trajectories, as shown in Figs 3-5 through 3-8. One must then select the theoretical trajectory that best matches the measured cross joint path, which in turn provides constraints on the remote principal stress ratio during cross joint formation.

For closed cases, one must first classify the observed cross joint geometry according to its quasi-curving or non-curving geometry. For non-curving geometries one can determine the range of the remote principal stress ration by using equation (3-36) and assuming a value for the coefficient of friction along the pre-existing joint. For quasi-curving geometries equation (3-38) can be employed along with constraints on remote stress ratio to plot several theoretical cross joint paths, as demonstrated in Figs 3-9, 3-10, 3-12, and 3-13. Once again, by comparing theoretical paths with the observed cross joint path, one can estimate the remote principal stress ratio that prevailed during cross joint propagation.

For cases where compressive zones exist near pre-existing joints, one can determine stress ratios using two methods: the cross joint trajectory method of equation (3-38) (i.e., "path fit method") and the equation describing compressive zone dimensions (equation 3-60) (i.e., 
"compressive zone dimension method"). This provides the opportunity to compare independently-derived values for the remote stress ratio.

The steps involved in estimating stress ratios from field examples are outlined in the flow chart of Fig. 4-1. Application of cross joint theory to joints measured in outcrop are provided in the following three field examples from California, Israel, and New York.

\section{EXAMPLE 1: CURVING PARALLEL AND CURVING- PERPENDICULAR CROSS JOINTS FROM THE MONTEREY FORMATION, CALIFORNIA}

The outcrop, referred to as Lompoc Landing, is located along the Pacific coastline in the southern Santa Maria Basin, California (Fig. 4-2). Figure 4-3 (a) is a photograph of a portion of the outcrop depicting several cross joints in the vicinity of a pre-existing joint exposed on a bedding plane surface of the Miocene Monterey Formation. The two dominant systematic joint sets in the Monterey Formation are both normal to bedding, with one parallel to the trend of regional fold axes and the other normal to fold axes (e.g., Dunham and Blake, 1987; Narr and Suppe, 1991; Gross, 1995). The flat-lying bed containing the cross joint is a dolomitic opal-CT porcellanite with a mechanical thickness of $22 \mathrm{~cm}$. By carefully sketching the photograph, one can measure the angular relationships between the initial cross joint trends and the trend of the pre-existing joint, as shown in Fig. 4-3 (b). Four individual cross joints were measured, with 


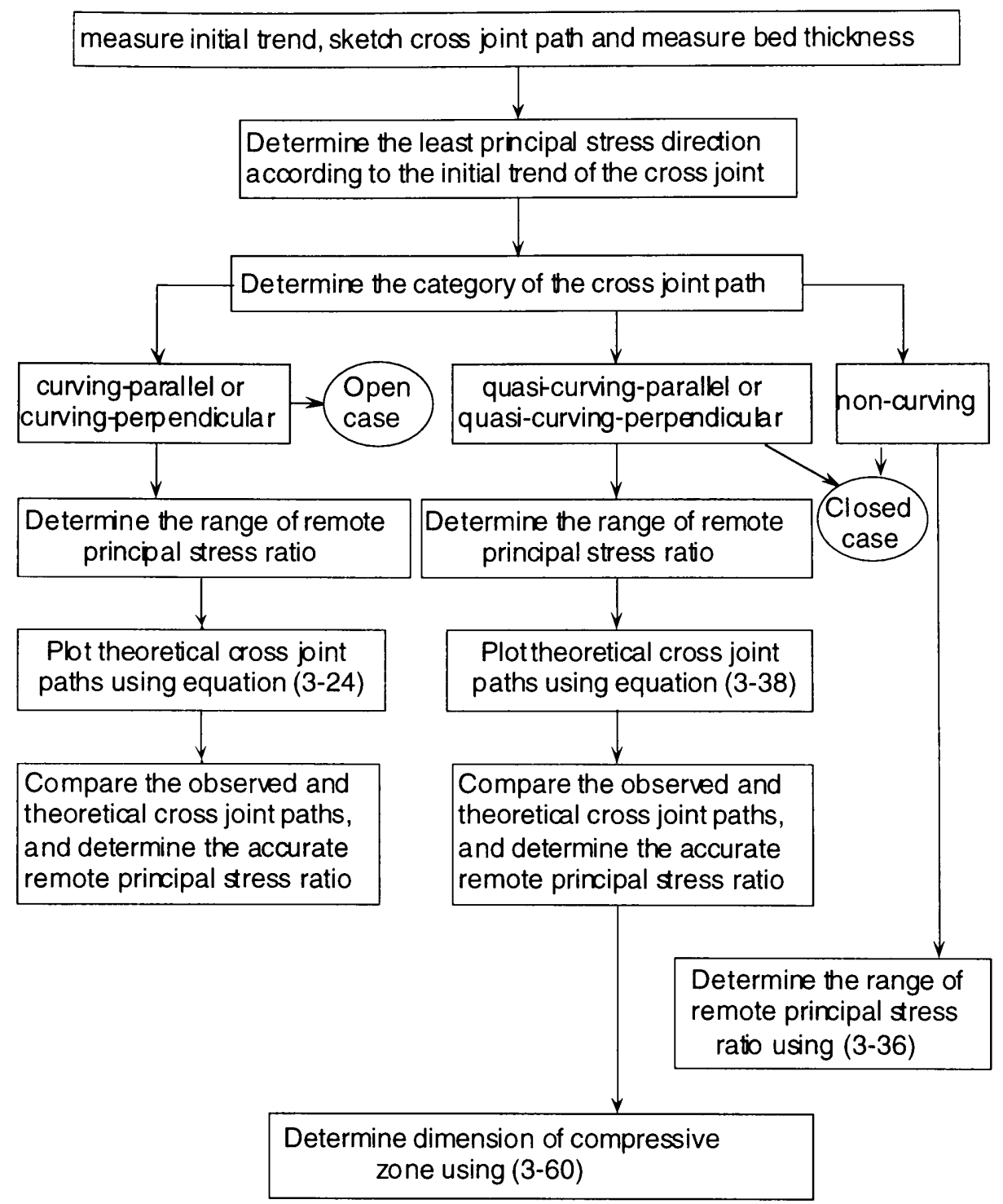

Fig. 4-1. Procedures for applying analytical solutions to cross joints observed in the field. 


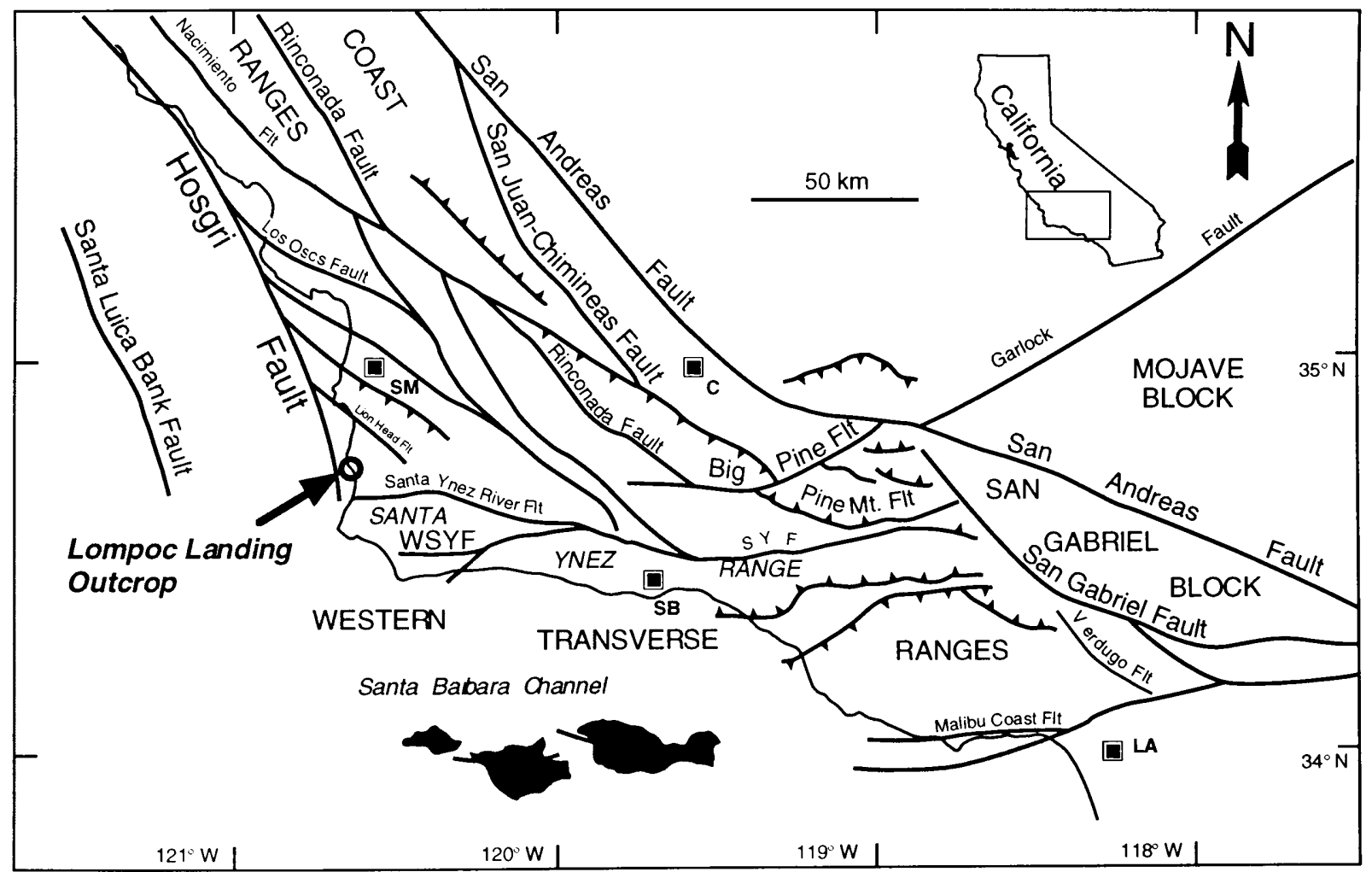

Fig. 4-2. Regional structural map of the western Transverse Ranges. (After Hornafius, 1985; Gross, 1993b). 

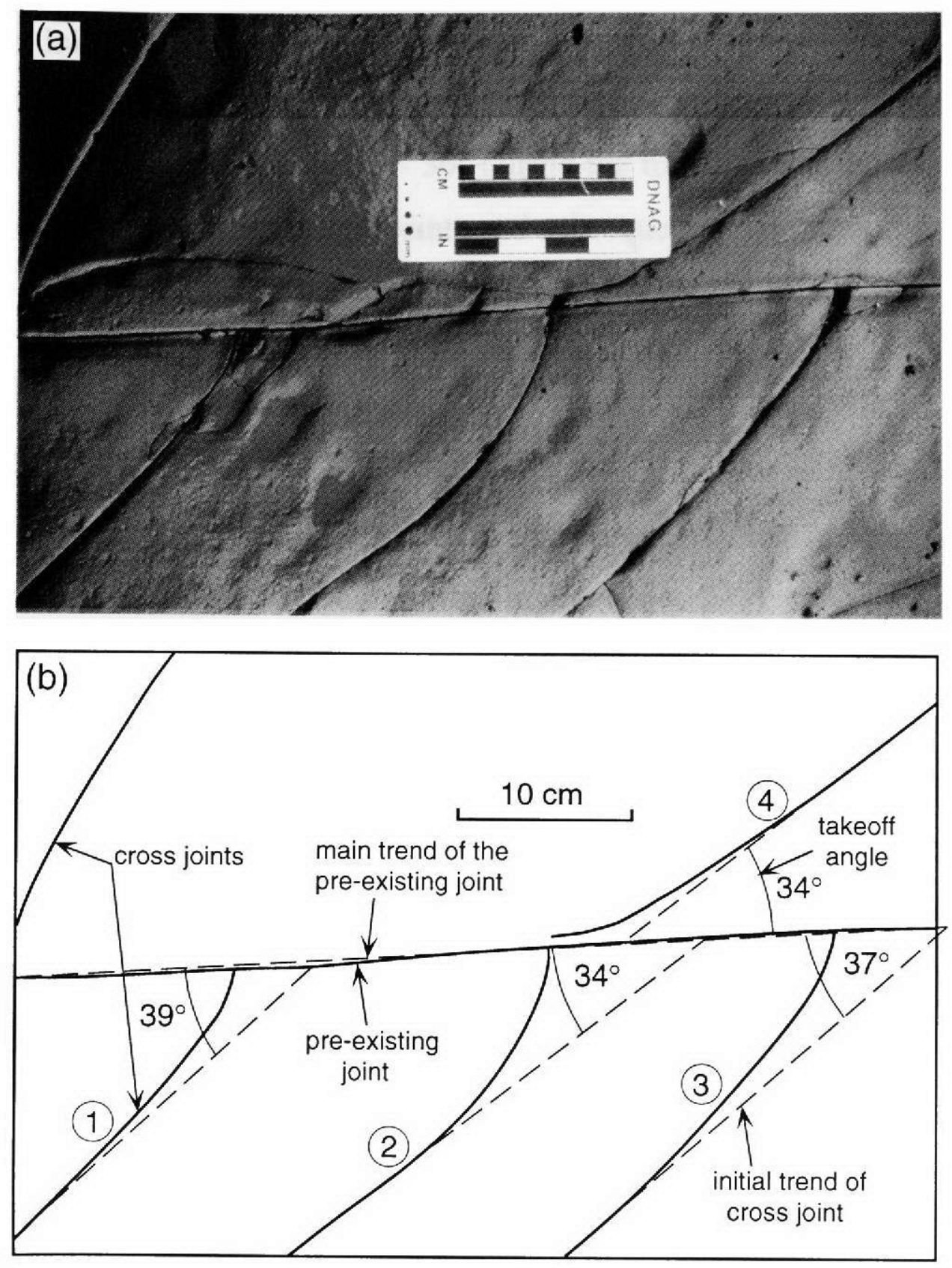

Fig. 4-3. (a) A photo of cross joint examples from the Monterey formation, California. (b) Sketch of the pre-existing joint and cross joints in the photo. Photo was provided by Dr. Michael Gross. 
take-off angles ranging from $34^{\circ}$ to $39^{\circ}$ and a mean of $36^{\circ}$. As stated above, initial cross joint trends are aligned parallel to the remote maximum horizontal principal stress orientation $\left(\sigma_{2}^{\circ}\right)$ during formation of the cross joints. Therefore, $\left(\sigma_{2}^{\infty}\right)$ rotated $\sim 36^{\circ}$ during the time interval between development of the systematic joints and subsequent cross joints.

\section{CROSS JOINT ANALYSIS}

Inspection of cross joint termination geometries indicate that joints $\# 1$, \#2, and \#3 belong to the curving-perpendicular category, and cross joint \#4 is curving-parallel (refer to Table 3-3). In all cases there is no evidence for a significant compressive zone. Therefore, one may conclude the pre-existing joint was open during formation of these cross joints. Based on curving geometries and the fact that all take-off angles are less than $45^{\circ}$, the cross joints can be further classified according to Table 3-3; joints \#1, \#2, \#3 belong to Open Case \#2, whereas joint \#4 belongs to Open Case \#1. Ranges in remote principal stress ratio can then be estimated, as listed in Table 4-1.

The next step is to construct cross joint paths based on take-off angle, bed thickness and range of remote principal stress ratio. For cross joint \#1 the calculated paths are shown in Fig. 4-4a. By comparing theoretical trajectories to the measured path for cross joint \#1, one concludes that the remote principal stress ratio was approximately -0.4 during formation of 
(a)

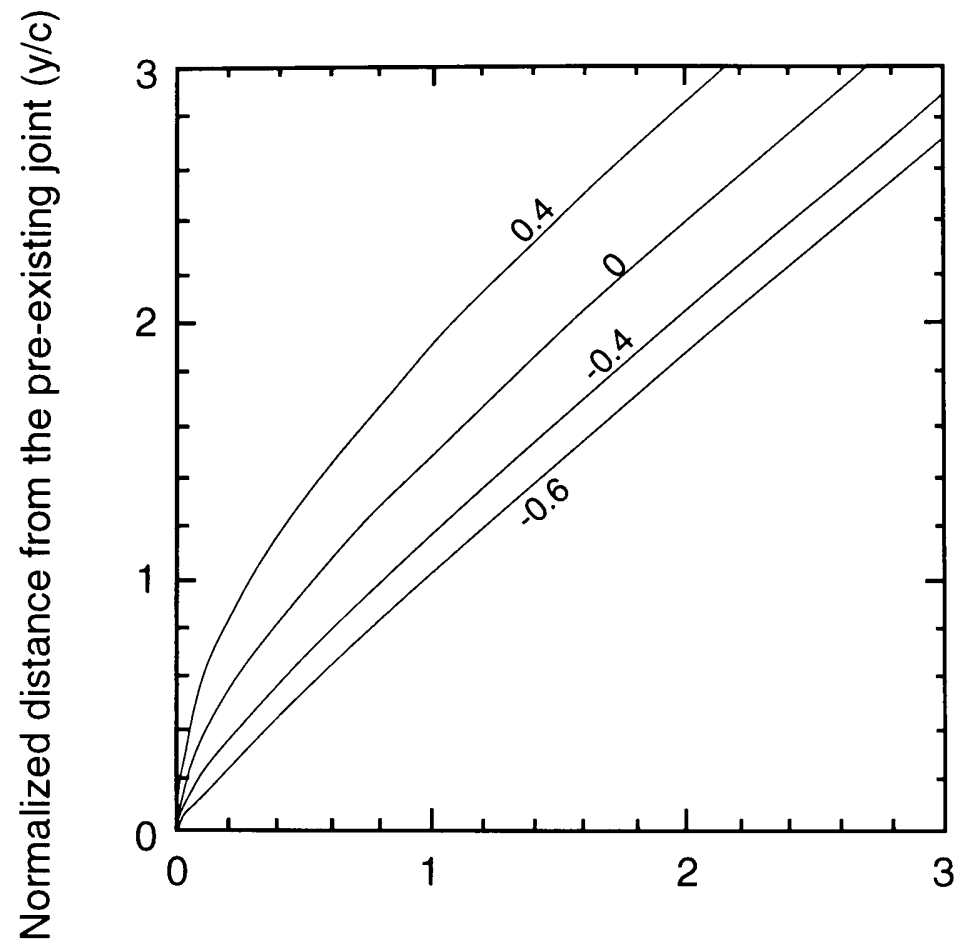

Normalized distance along the pre-existing joint $(z / c)$

(b)

observed cross

joint path (cross joint \#1

in Fig. 4-2) calculated cross joint path (take off angle $=39^{\circ}$ remote principal stress ratio $=-0.4$

Fig. 4-4. (a) Calculated cross joint paths for different remote principal stress ratios with a takeoff angle of $39^{\circ}$. (b) Comparison of observed and calculated cross joint paths. Note that the observed cross joint path is very closed to the calculated cross joint path with a takeoff angle of $39^{\circ}$ and a remote principal stress ratio of -0.4 . 
(a)

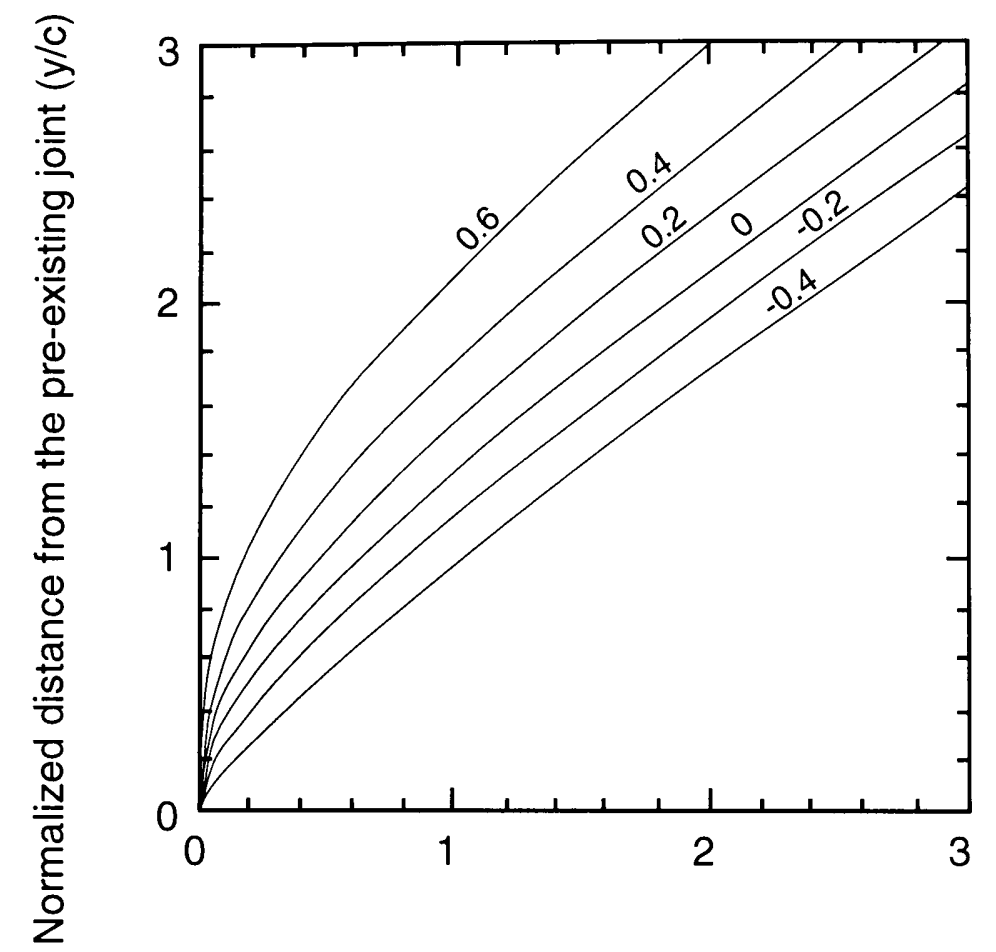

Normalized distance along the pre-existing joint $(\mathrm{z} / \mathrm{c})$

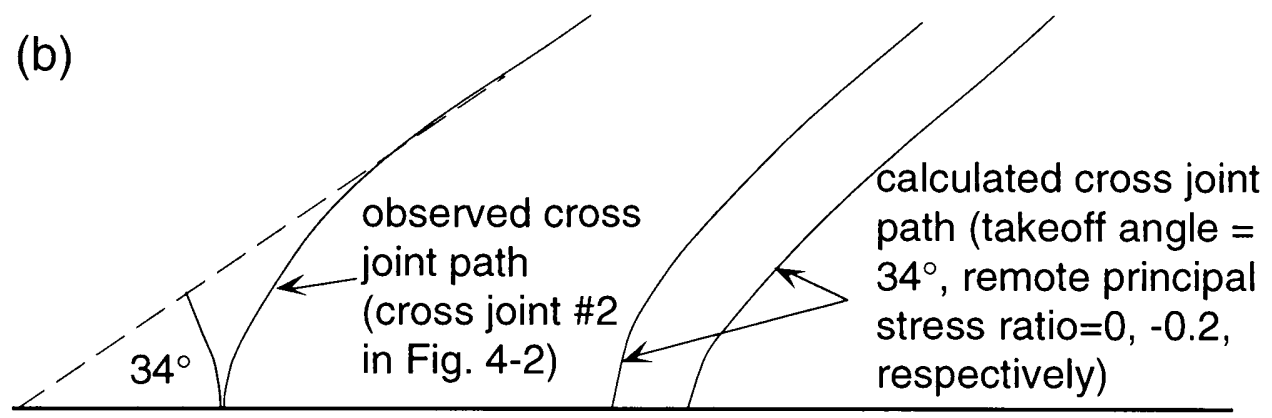

Fig. 4-5. (a) Calculated cross joint paths for different remote principal stress ratios with a takeoff angle of $34^{\circ}$. (b) Comparison of observed and calculated cross joint paths. In this example the observed cross joint path and the calculated cross joint paths have a greater discrepancy than that in Figs. 4-3 and 4-5. 
(a)

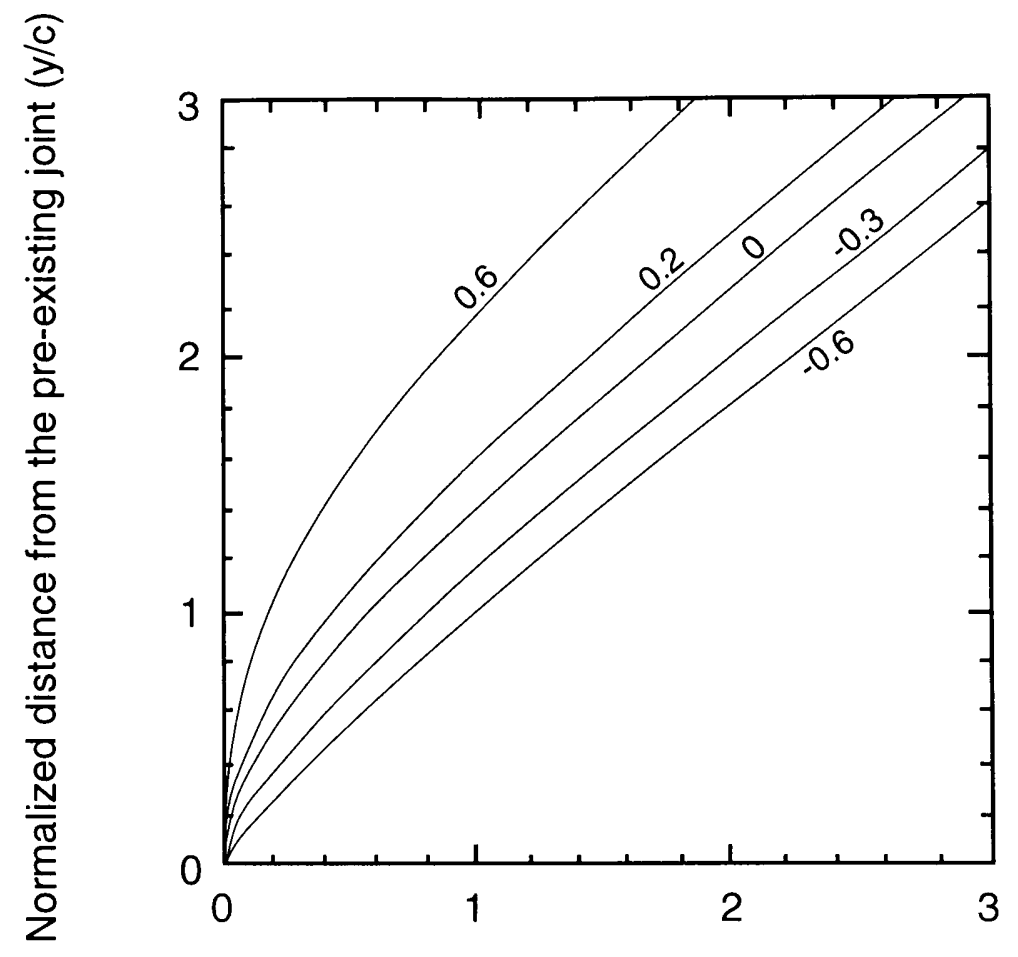

Normalized distance along the pre-existing joint $(z / c)$

(b)

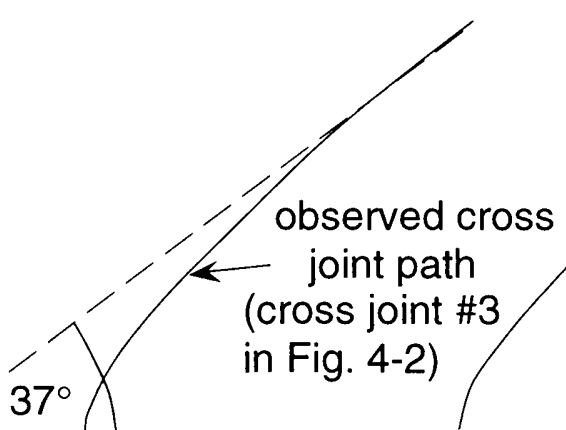

calculated cross joint path (takeoff nagle $=37^{\circ}$, remote principal stress ratio $=-0.3$ )

Fig. 4-6. (a) Calculated cross joint paths for different remote principal stress ratios with a takeoff angle of $37^{\circ}$. (b) Comparison of observed and calculated cross joint paths. Note that the observed cross joint path closely matches the calculated cross joint path with a takeoff angle of $37^{\circ}$ and a remote principal stress ratio of -0.3 . 
(a)

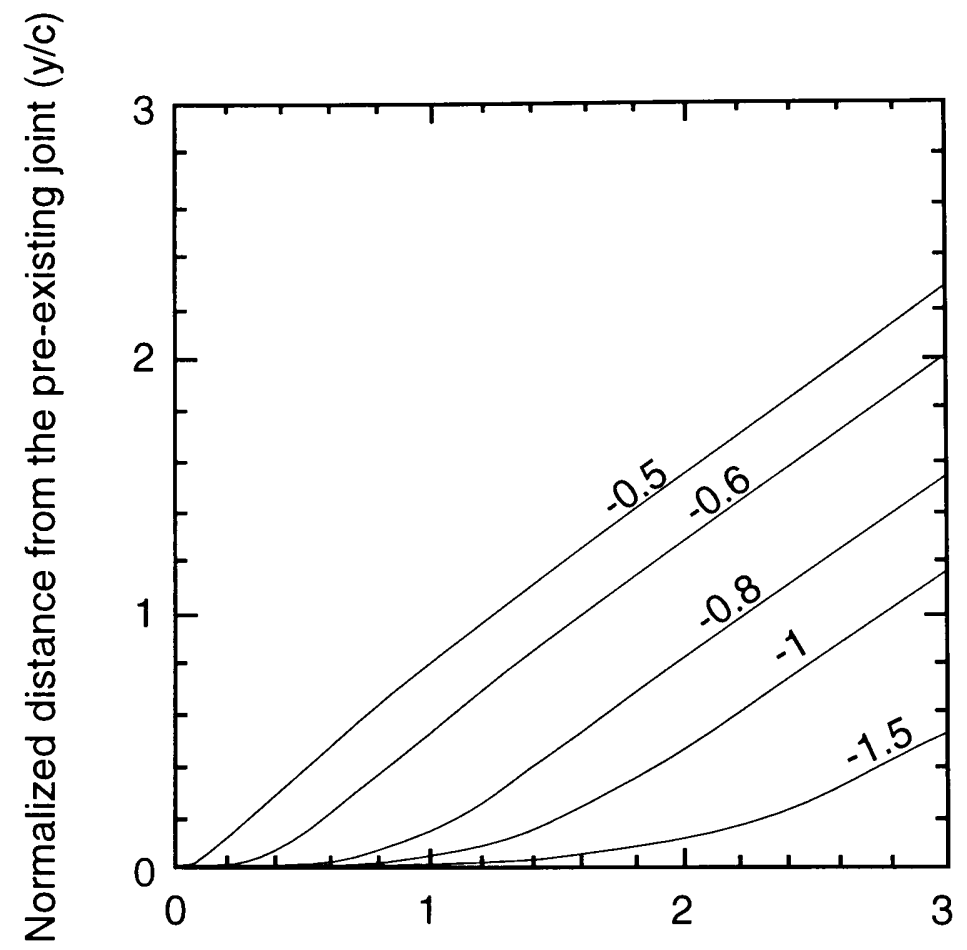

Normalized distance along the pre-existing joint $(\mathrm{z} / \mathrm{c})$

(b)

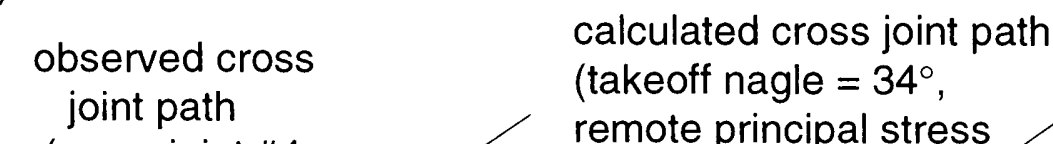
(cross joint \#4 in Fig. 4-2)

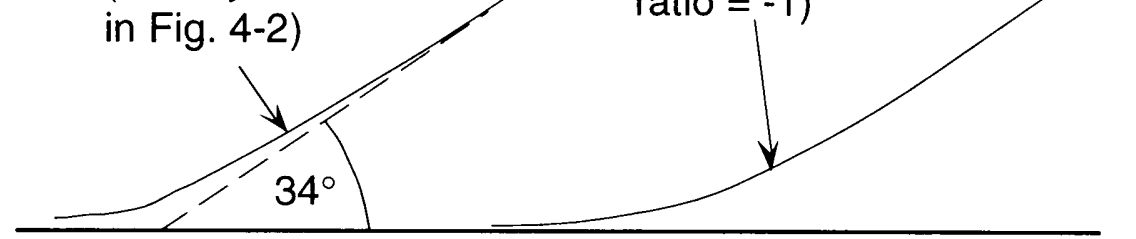

Fig. 4-7. (a) Calculated cross joint paths for different remote principal stress ratios with a takeoff angle of $34^{\circ}$. (b) Comparison of observed and calculated cross joint paths. In this example the observed cross joint path is very close to the calculated cross joint paths with a takeoff angle of $34^{\circ}$ and a remote principal stress ratio of -1 . 
Table 4-1. Principal stress ratios estimated from the cross joint examples in Fig. 4-2.

\begin{tabular}{|c|c|c|c|c|}
\hline $\begin{array}{c}\text { Cross joint } \\
\text { \# in } \\
\text { Fig. } 3-1\end{array}$ & 1 & 2 & 3 & 4 \\
\hline $\begin{array}{l}\text { Takeoff } \\
\text { angle }\left({ }^{\circ}\right)\end{array}$ & 39 & 34 & 37 & 34 \\
\hline $\begin{array}{c}\text { Main } \\
\text { category }\end{array}$ & $\begin{array}{c}\text { Open Case } \\
\# 2\end{array}$ & $\begin{array}{c}\text { Open Case } \\
\# 2\end{array}$ & $\begin{array}{c}\text { Open Case } \\
\# 2\end{array}$ & $\begin{array}{c}\text { Open Case } \\
\# 1\end{array}$ \\
\hline $\begin{array}{l}\text { Range of } \\
\text { effective } \\
\text { principal } \\
\text { stress } \\
\text { ratio }\left(\eta^{*}\right)\end{array}$ & $-0.66<\eta^{*}<1$ & $-0.45<\eta^{*}<1$ & $-0.57<\eta^{*}<1$ & $-2.20<\eta^{*}<-0.45$ \\
\hline $\begin{array}{c}\text { Effective } \\
\text { principal } \\
\text { stress } \\
\text { ratio }\left(\eta^{*}\right)\end{array}$ & -0.4 & $0.0 \sim-0.2$ & -0.3 & -1.0 \\
\hline
\end{tabular}


the cross joint (Fig. 4-4b). The same procedure was followed for the other cross joints. Theoretical paths for cross joints \#2, \#3, and \#4 are shown in Fig. 4-5a, 4-6a, and 4-7a, respectively. Comparison of theoreticallyderived trajectories with measured traces of cross joints \#3 and \#4 also yield excellent matches (Figs. 4-6b and 4-7b). Estimated effective principal stress ratios that prevailed during the formation of cross joints \#3 and \#4 are -0.3 and -1.0 , respectively (Table $4-1$ ). In contrast, the same procedure applied to cross joint \#2 does not provide an accurate match. At best one can place cross joint \#2 within a stress ratio range of 0 to -0.2 (Fig. 4-5b).

\section{INTERPRETATION OF CROSS JOINTS IN THE MONTEREY FORMATION}

The application of theoretical cross joint analysis to the example from the Monterey Formation in Fig. 4-3 leads to several results that require further interpretation. One question to address is why does cross joint \#2 differ from adjacent cross joints \#1 and \#3? One must also address the geologic significance of the different stress ratios derived from curving perpendicular $\left(\eta^{*} \sim-0.3\right)$ and curving-parallel cross joints $\left(\eta^{*} \sim 1.0\right)$.

The difficulty in finding a good match between theoretical trajectories and the measured path for cross joint \#2 may arise because the assumption of no interaction between adjacent cross joints no longer applies. Like other mechanically-confined systematic joints, sets of cross joints develop through a process of "sequential infilling", whereby new 
joints form in between existing joints (e.g., Hobbs, 1967; Gross, 1993a \& b). Evidence for sequential infilling is provided by different amounts of vein filling in boudinaged piedmontite grains (Masuda and Kuriyama, 1988; Masuda et al, 1989, 1990), as well as alternating curving and noncurving cross joints (Engelder and Gross, 1993). Because paths of cross joints \#1 and \#3 match theoretical trajectories, it appears they propagated as if in a homogeneous medium. If cross joint \#2 formed during infilling subsequent to joints \#1 and \#3, then its development may have been influenced by the presence of pre-existing cross joints. In fact, Engelder and Gross (1993) demonstrate that when cross joint spacing is relatively small, late-forming cross joints are indeed affected by interaction with pre-existing cross joints. Therefore, I conclude that cross joint \#2 formed subsequent to cross joints \#1 and \#3 during infilling, and its deviation from theoretically-predicted paths is due to mechanical interaction with adjacent pre-existing cross joints. It should also be noted that the estimated value for stress ratio from cross joint \#2 (i.e., $0<\mathrm{h}<-0.2$ ) is close to the values derived from cross joints \#1 and \#3.

Because cross joint \#4 is curving-parallel, it formed under markedly different stress conditions than cross joints \#1 and \#3; for curvingperpendicular joints (i.e., \#1, \#2, and \#3) both the stresses normal and parallel to the pre-existing joint were tensile, whereas for curving-parallel cross joints (i.e., \#4) the stress normal to the joint was tensile but parallel to the pre-existing joint the stress was compressive during propagation. One may note that with respect to cross joints \#1-\#3, cross joint \#4 is on the opposite side of the pre-existing joint. This means that the timing of 
propagation for \#4 relative to the other cross joints is unclear. However, one cannot reach the general conclusion that there is a switch in geometry from parallel to perpendicular across the pre-existing joint; both styles of cross joint termination are found on either side. In fact the cross joint adjacent to \#4 (top left corner of Fig. 4-3 a, b) has a curving-perpendicular geometry. Therefore, an explanation must be found that accounts for changes in cross joint geometry, and hence principal stress ratios, at this locality.

The take-off angle for cross joints \#2 and \#4 is $34^{\circ}$, whereas for cross joints \#1 and \#3 the angle is slightly larger at $39^{\circ}$ and $37^{\circ}$, respectively. It is thus possible that cross joints \#2 and \#4 propagated after a slight reorientation of the remote stress field that formed joints \#1 and \#3. However, it is my opinion that the differences in take-off angles are too small to resolve, and hence I do not attribute these small differences to changes in remote stress orientation. Rather, I assume a uniformly oriented remote stress field throughout development of curvingperpendicular and curving-parallel cross joints.

There are two possible geologic scenarios that may give rise to the cross joint pattern observed in the outcrop of Monterey Formation along the Santa Maria coastline. One explanation considers the effects of fluctuating pore pressure on effective stress ratio. If the magnitudes of $\sigma_{2}^{\infty}$ and $\sigma_{3}^{\infty}$ remain constant, the effective stress ratio can change due to fluctuation in pore pressure, as shown in Fig. 4-8a. In terms of Mohr space, this would translate a Mohr's circle of fixed diameter along the horizontal (e.g., normal stress) axis (Fig. 4-8b). For the general outcrop 

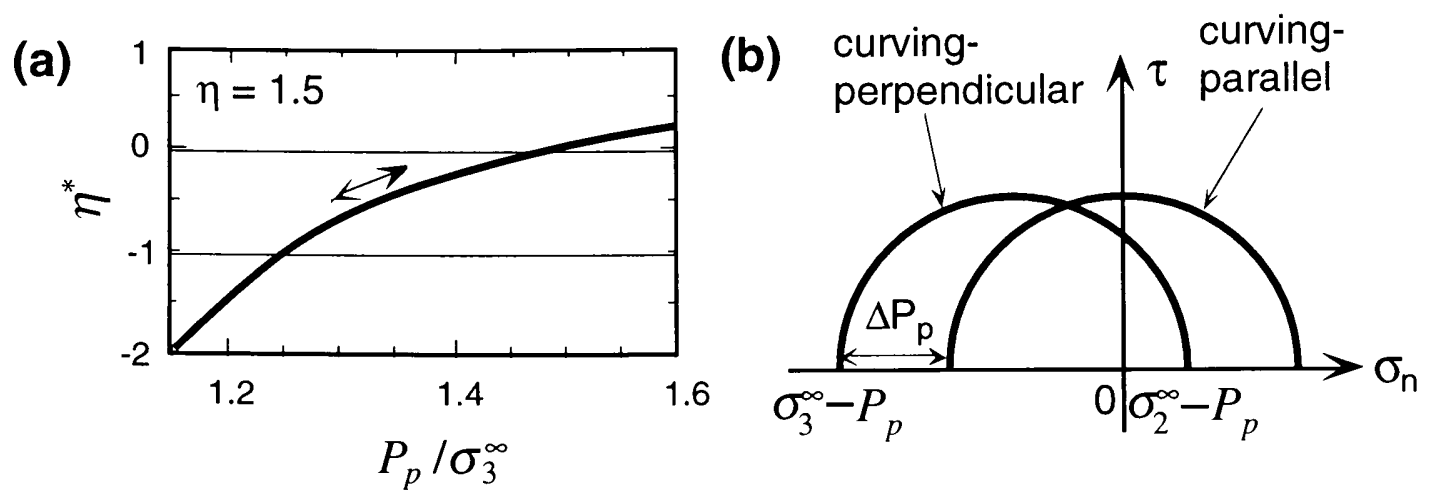

(c) curving-perpendicular $\uparrow \tau$ curving-parallel

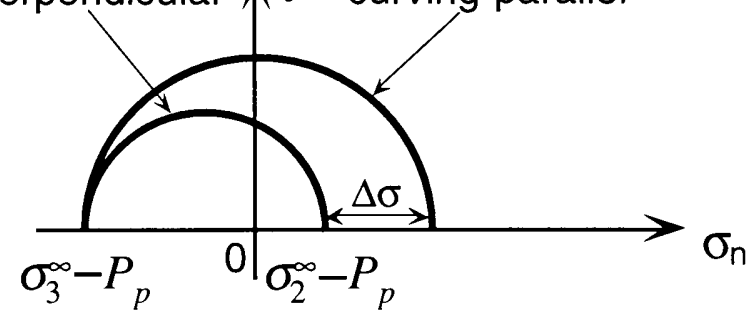

Fig. 4-8. (a) Plot of effective remote principal stress ratio $\left(\eta^{*}\right)$ versus fluid pressure/least principal stress ratio $\left(\mathrm{P}_{\mathrm{p}} / \sigma_{3}^{\infty}\right)$ at a constant remote principal stress ratio $(\eta)$. (b) Mohr circle diagram showing stress state variation as a function of fluid pressure $\left(\mathrm{P}_{\mathrm{p}}\right)$ with constant remote principal stresses $\left(\sigma_{2}^{\infty}\right.$ and $\left.\sigma_{3}^{\infty}\right)$. Note that curvingperpendicular geometry occurs at higher fluid pressure and curvingparallel geometry occurs at lower fluid pressure. (c) Mohr circle diagram showing stress state variation as a function of maximum horizontal stress $\left(\sigma_{2}^{\infty}\right)$ with constant minimum horizontal stress $\left(\sigma_{3}^{\infty}\right)$ and fluid pressure $\left(\mathrm{P}_{\mathrm{p}}\right)$. Note that curving-perpendicular geometry occurs at lower $\sigma_{2}^{\infty}$ and curving-parallel geometry occurs at higher $\sigma_{2}^{\infty}$. 
stress conditions of $\eta^{\circ}<0$ (see Table 4-1), cross joints with curvingperpendicular geometries would form when $\left|\sigma_{3}^{\infty}-\mathrm{P}_{\mathrm{p}}\right|>\left|\sigma_{2}^{\infty}-\mathrm{P}_{\mathrm{p}}\right|$, whereas curving-parallel cross joint would develop when $\left|\sigma_{3}^{\infty}-\mathrm{P}_{\mathrm{p}}\right|=\left|\sigma_{2}^{\infty}-\mathrm{P}_{\mathrm{p}}\right|$. As shown in the Mohr diagram in Fig. 4-8b, the curving-perpendicular cross joints develop under higher fluid pressures than the curving-parallel cross joints.

Joints often grow in stages, with increments of extension followed by periods of non-growth. Evidence for such incremental joint growth is provided by plumose structures often preserved on joint surfaces (e.g., Kulander et al, 1985; Bahat and Engelder, 1984; Lacazette and Engelder, 1992; Wu and Pollard, 1995). Each increment of growth is marked by an arrest line indicating the point of temporary cessation of joint growth (Fig. 4-9a). As mentioned earlier, fluid pressure plays a key role in enabling joints to propagate under compressive stress conditions (e.g., Secor, 1965; Segall and Pollard, 1983; Engelder and Lacazette, 1990). Consequently, the series of arrest lines often found on single joint surfaces are thought to represent cyclic joint propagation due to fluctuations in fluid pressure (fig. 4-9b) (Lacazette and Engelder, 1992). In other words, when fluid pressure within the joint reaches a critical value, the joint extends a certain distance until the internal fluid pressure drops. The drop in fluid pressure occurs because the quantity of fluid is now distributed across a larger volume. This leads in turn to a decrease in pore pressure in the surrounding rock as fluid moves from a zone of higher pressure (i.e., surrounding rock) to a zone of lower pressure (i.e., the joint). Thus, fluid 


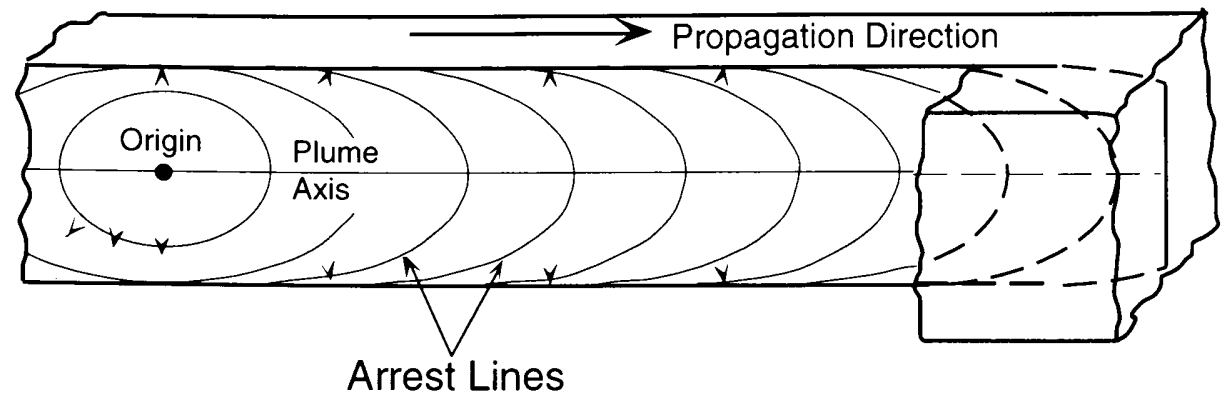

(a)

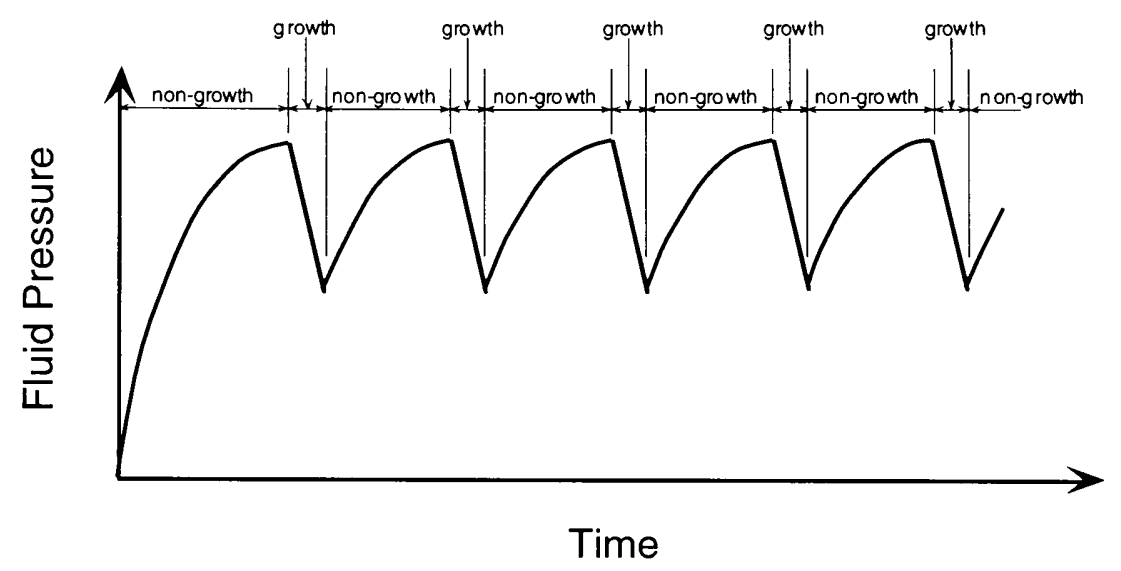

(b)

Fig. 4-9. (a) Block diagram of a joint surface showing arrest lines, plume axis and fracture propagation direction. Arrest lines are indicators of temporary cessation of joint growth (Redrawn from Kulander et al, 1990). (b) Fluid pressure variation through time during incremental joint propagation. 
pressure cycles through time as shown in Fig. 4-9b, at a rate controlled by the permeability of the host rock and the mechanism for creating overpressured conditions.

It is certainly possible that pore pressure fluctuated during development of cross joints in the Monterey Formation, as the pre-existing joints could have served as a plumbing mechanism for transporting fluid into and out of the rock system. For the cross joints observed in Fig. 4-3a, curving-perpendicular joints would have formed under higher pore pressures, whereas curving-parallel joints would have formed under lower pore pressures. Due to fluctuations in pore pressure magnitude under this scenario, one would expect alternating geometries to develop throughout the history of formation of the cross joint set.

The second possible explanation for the observed cross joint pattern is termed the "tectonic stress effect", and considers the case where $\sigma_{3}^{\infty}$ and $P_{p}$ remain constant through time. Under this scenario, the Mohr's circle of stress remains anchored on the left side as shown in Fig. 4-8c. Curvingperpendicular geometries form under low differential stress, where $\left|\sigma_{3}^{\infty}-\mathrm{P}_{\mathrm{p}}\right|>\left|\sigma_{2}^{\infty}-\mathrm{P}_{\mathrm{p}}\right|$. An increase in maximum horizontal stress (in this case $\left.\sigma_{2}^{\infty}\right)$, perhaps due to intensification of tectonic shortening across Santa Maria Basin (e.g., Namson and Davis, 1990), would lead to an increase in differential stress, and hence a Mohr's circle with larger diameter. Curving-parallel cross joints would then form under conditions of higher differential stress when $\left|\sigma_{3}^{\infty}-\mathrm{P}_{\mathrm{p}}\right|=\left|\sigma_{2}^{\infty}-\mathrm{P}_{\mathrm{p}}\right|$. In contrast to the pore pressure fluctuation model, under this scenario one would expect a switch from 
curving-perpendicular cross joint propagation to curving-parallel development through time.

\section{EXAMPLE 2: A QUASI-CURVING-PARALLEL CROSS JOINT FROM THE GEROFIT FORMATION, ISRAEL}

From Table 3-3 we can see that quasi-curving-parallel cross joints belong to the Closed Case \#1. The typical features of the Closed Case \#1 are such that cross joints are not exactly parallel to the pre-existing joint, and there are compressive zones in the vicinity of the pre-existing joint. Theoretically, cross joints cannot propagate into a compressive zone (Dyer, 1988). Therefore, cross joint termination can be used as an indicator of the compressive zone boundary. In example 1 I showed a method of estimating the remote effective principal stress ratio by fitting the observed cross joint paths with the theoretical cross joint paths, which is called the path-fit method. In this example, I will demonstrate a method of using the dimension of the compressive zone to estimate remote effective principal stress ratio, which I refer to as the compressive zone dimension method.

Figure 4-10a shows a picture of a cross joint and a pre-existing joint developed in a marly limestone unit with a bed thickness of $6 \mathrm{~cm}$ in the Gerofit Formation of Israel. The outcrop is located on the southern flank of the Haluza Anticline in the vicinity of the Zin Fault (Fig. 4-11). The Haluza Anticline is an open drape-fold with maximum limb dips of about 


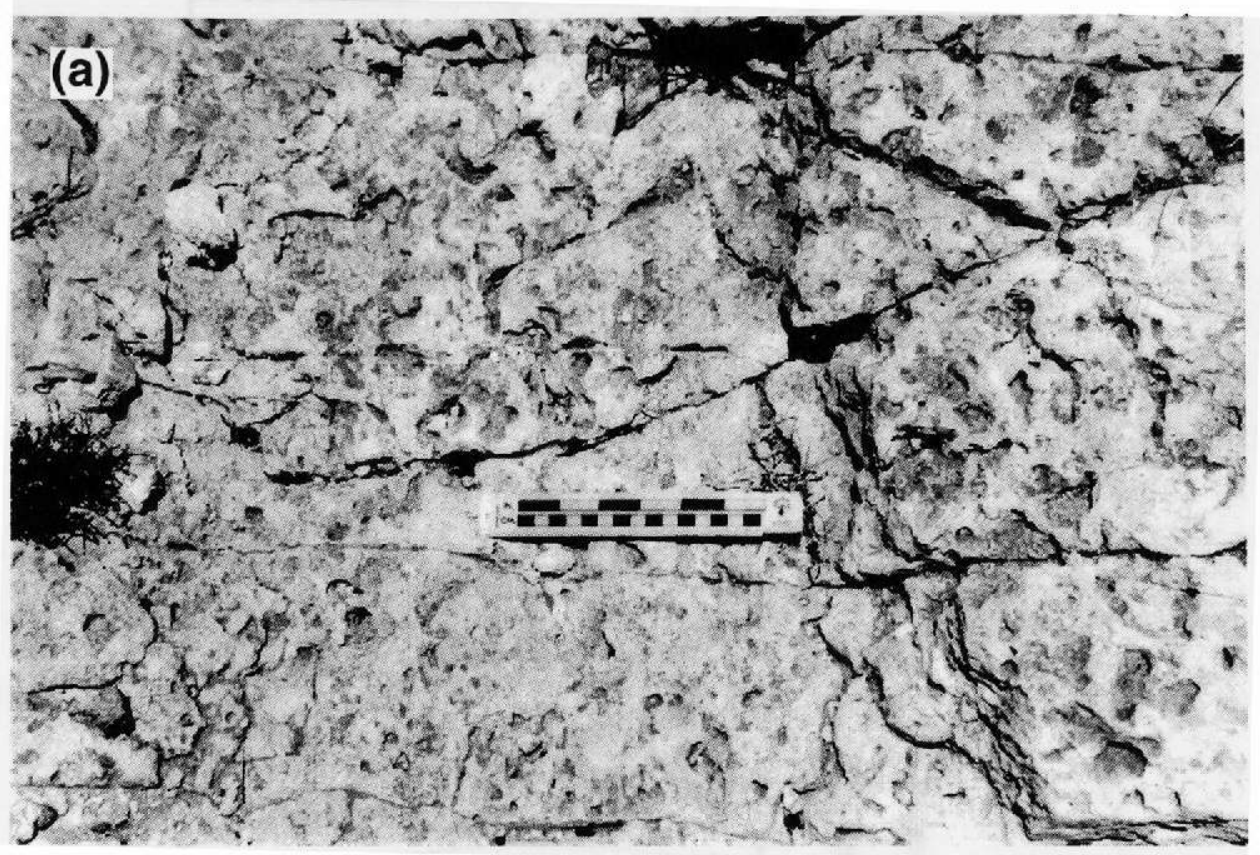

(b)

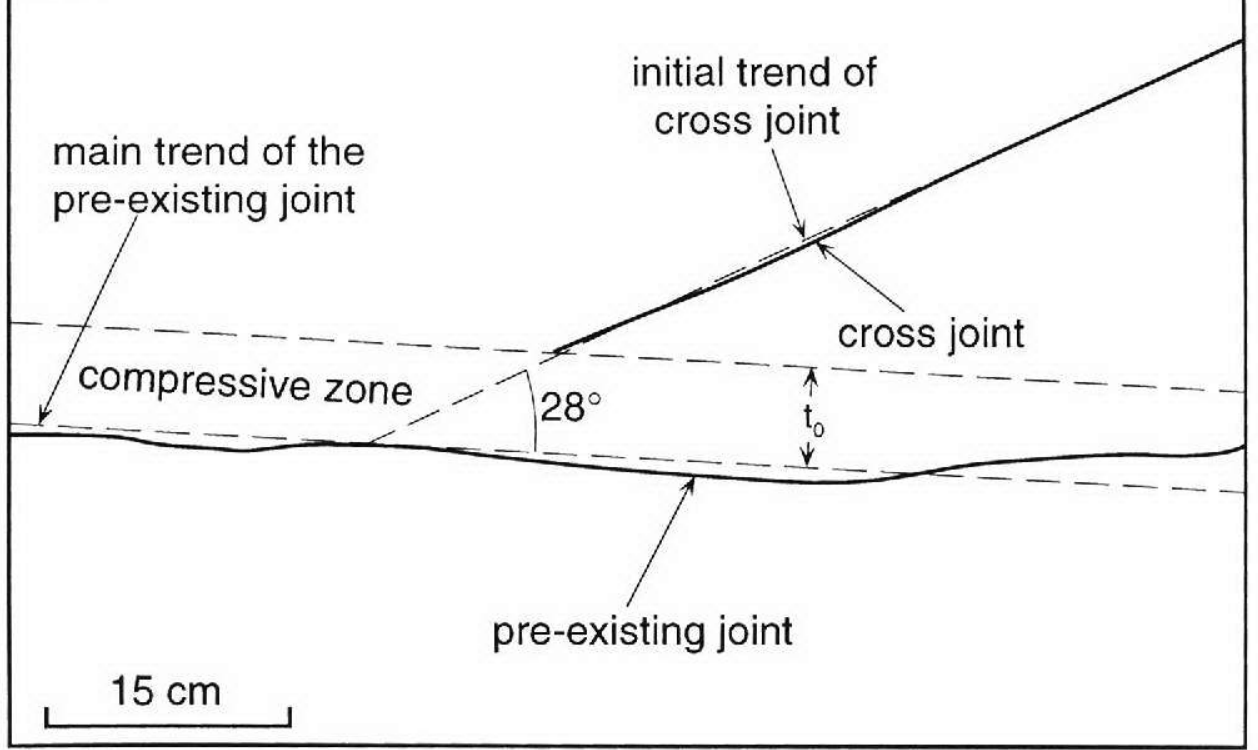

Fig. 4-10. (a) A photo of cross joint example from Israel. (b) Sketch of the pre-existing joint and the cross joint in the photo. Photo taken by Dr. Alexander Beker. 


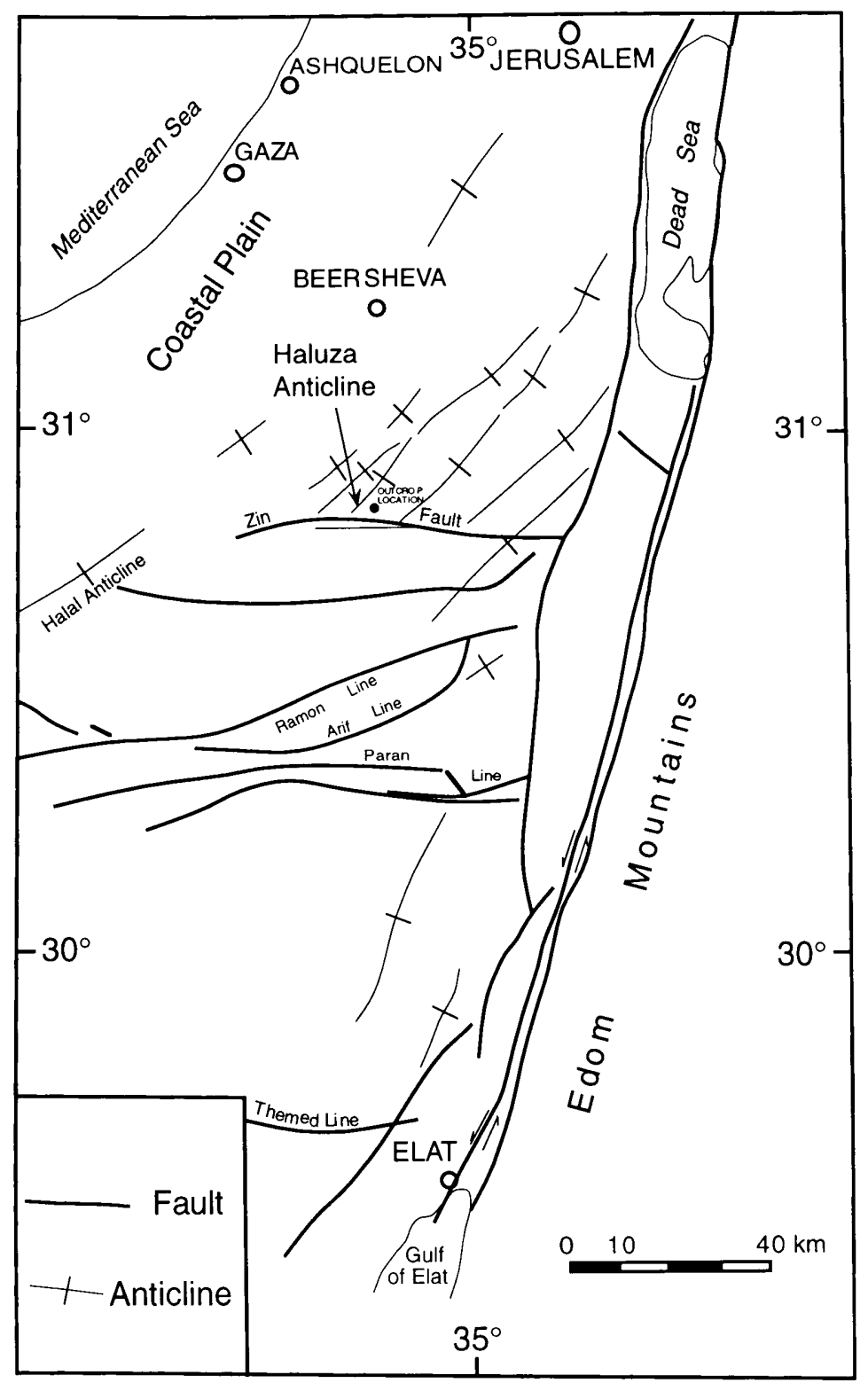

Fig. 4-11. A simplified structural map of Southern Israel showing the locations of Zin Fault and Haluza Anticline. Redrawn from Garfunkel (1978). 
$30^{\circ}$. The Zin Fault is a steep reverse fault belonging to the Syrian Arc Fault System.

Figure $4-10 \mathrm{~b}$ is the sketch of the photo in Fig. 4-10a. The angle between the initial trend of the cross joint and the main trend of the preexisting joint shows that the horizontal principal stress direction during cross joint formation lies in the direction of $28^{\circ}$ (i.e., the take off angle) from the pre-existing joint. From the equations provided in Table 3-3 and using a take off angle of $28^{\circ}$, one estimates that the remote effective principal stress ratio during the formation of the cross joint was less than -3.54 .

Now let us use the dimension of the compressive zone to estimate the remote effective principal stress ratio more accurately. Using a take off angle of $28^{\circ}$ and assuming the coefficient of friction along the pre-existing joint is $0,0.6$ and 0.9 , respectively, we can plot the dimension of the compressive zone versus the remote effective principal stress ratio with equation (3-60) in chapter 3. The plots are shown in Fig. 4-12. It was mentioned above that cross joint termination can be used as an indicator of the compressive zone boundary. Using this criterion we can draw the boundary of the compressive zone in our examples, which is shown in Fig. $4-10 \mathrm{~b}$. The dimension of the compressive zone in the example is $6 \mathrm{~cm}$ (Fig. 4-10b). By normalizing the dimension of the compressive zone using the half bed thickness of $3 \mathrm{~cm}$, we derive a normalized dimension of the compressive zone of 2. Using this number, we can find the corresponding remote effective principal stress ratios from Fig. 4-12, which are -27.0, 


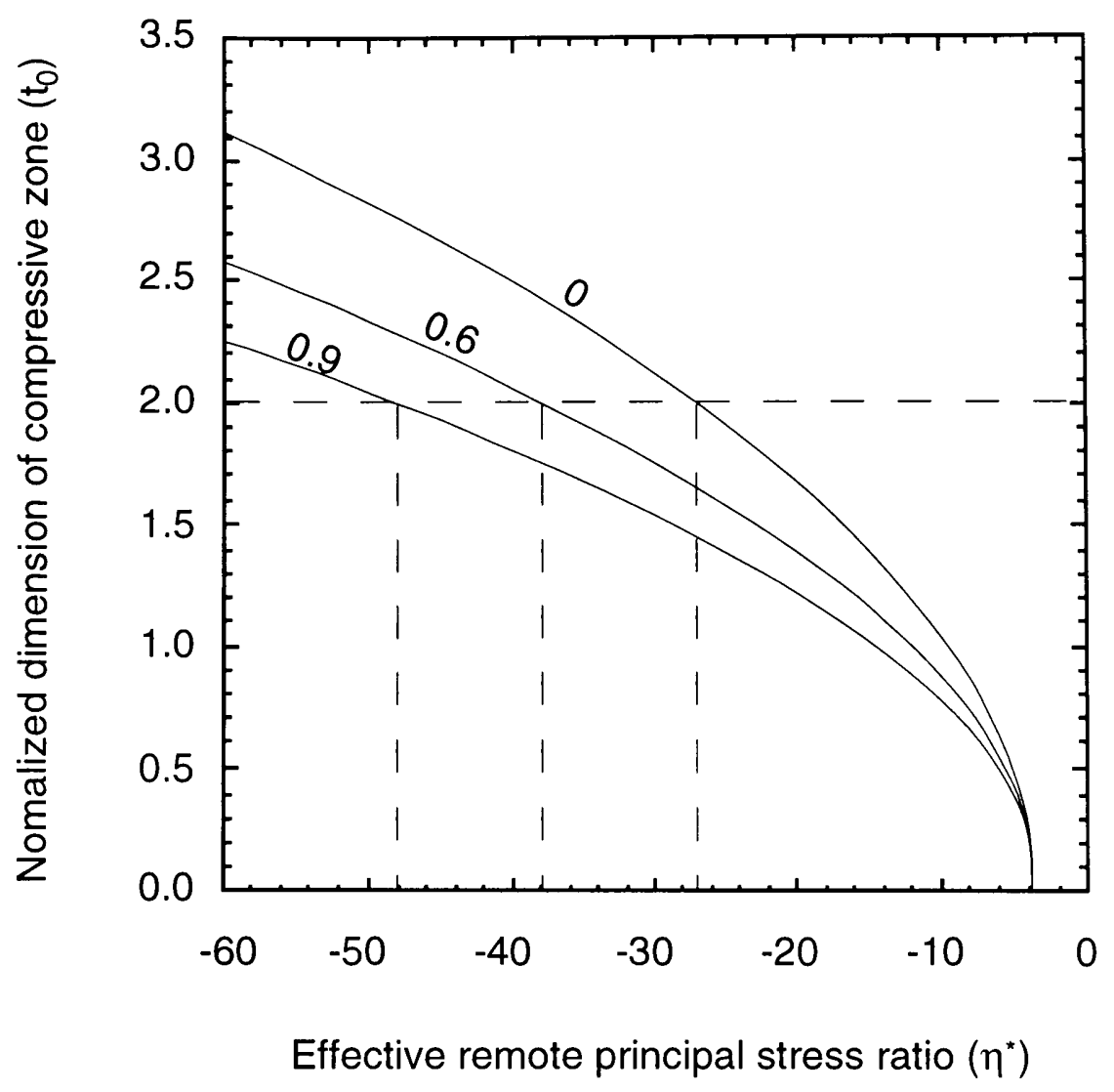

Fig. 4-12. Plots of dimension of compression versus remote effective principal stress ratio for Closed Case \#1 (Table 2-3) with a take off angle of $28^{\circ}$. The number next to each curve indicate the coefficient of friction along the pre-existing joint. 
-37.9 and -48.3 , corresponding to coefficients of friction of $0.0,0.6$ and 0.9 .

To verify this result, let us plot the theoretical cross joint paths based on the parameters from our example using equation (3-38). Figure 4-13a shows the calculated cross joint paths with effective remote principal stress ratios in the range of $-26 \sim-50$. The cross joint path change as a function of effective remote principal stress ratio is not detectable in the prescribed range of effective remote principal stress ratio. By comparing the theoretical cross joint path with the observed cross joint path in Fig. 4-10, we find that theoretical cross joint paths with remote effective principal stress ratios greater than -50 and less than -26 can fit the observed cross joint path (Fig. 4-13b). This result is in agreement with the result derived from the compressive zone dimension method, though the compressive zone dimension method yields more accurate estimations. The results are summarized in Table 4-2.

From the above discussion, we can see that the compressive zone dimension method is relatively easy to use because the only thing one needs to do is to plot the relationship between the dimension of compressive zone and the remote effective principal stress ratio. This method can also be used in Closed Case \#2aI (refer Table 3-3), which is the quasi-curvingperpendicular case with a compressive zone. 
(a)
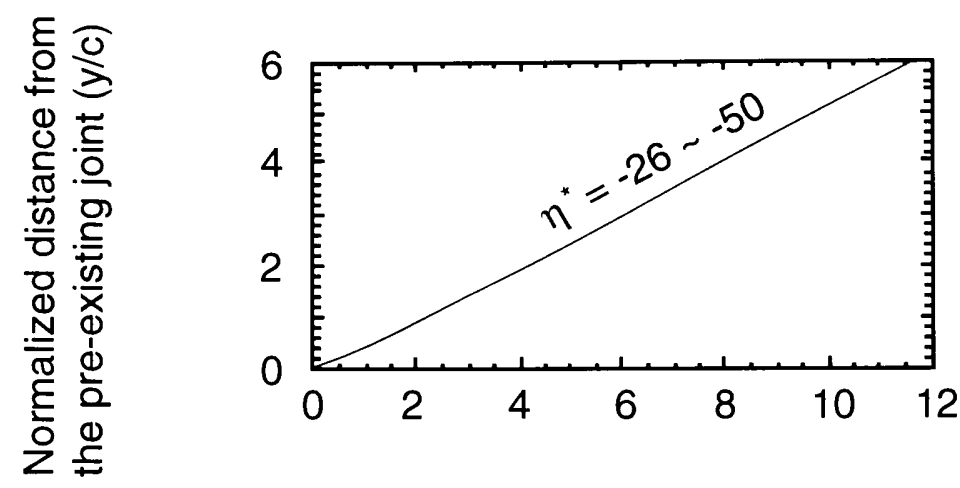

Normalized distance along the pre-existing joint $(z / c)$

(b)

calculated cross joint path (take off angle $=28^{\circ}$, remote effective principal stress ratio $=-26 \sim-50$ )

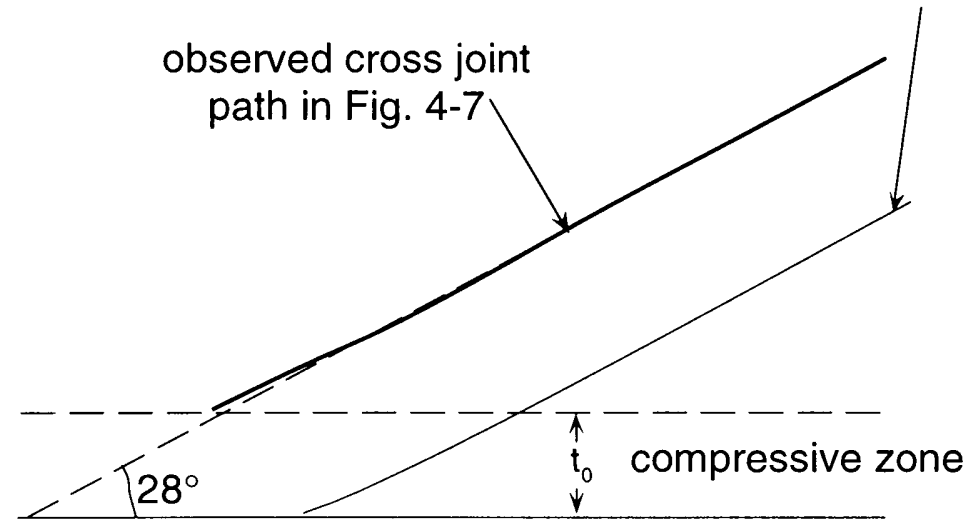

Fig. 4-13. (a) Calculated cross joint paths for different remote principal stress ratios with a takeoff angle of $28^{\circ}$. (b) Comparison of observed and calculated cross joint paths. In this example the observed cross joint path is very close to the calculated cross joint path with a remote principal stress ratio of -5.0 . 
Table 4-2. Remote effective principal stress and pre-existing joint information obtained from the cross joint in Fig. 4-7.

\begin{tabular}{|c|c|c|c|}
\hline & \multicolumn{3}{|c|}{ Cross joint in Fig. 4-7 } \\
\hline Take off angle $\left(^{\circ}\right)$ & \multicolumn{3}{|c|}{28} \\
\hline Main category & \multicolumn{3}{|c|}{ Closed Case \#1 in Table 3-3 } \\
\hline $\begin{array}{c}\text { Range of the remote effective } \\
\text { principal stress ratio }\left(\eta^{*}\right)\end{array}$ & \multicolumn{3}{|c|}{$\eta^{*}<-3.54$} \\
\hline \multirow{2}{*}{$\begin{array}{l}\text { Remote effective principal stress } \\
\text { ratio } \\
\left(\eta^{*}\right) \text { from the Compressive Zone }\end{array}$} & $C_{0}=0.0$ & $C_{0}=0.6$ & $C_{0}=0.9$ \\
\hline & -27.0 & -37.9 & -48.3 \\
\hline Dimension Method & & & \\
\hline $\begin{array}{c}\text { Remote effective principal stress } \\
\text { ratio }\left(\eta^{*}\right) \text { from the Path-fit } \\
\text { Method }\end{array}$ & \multicolumn{3}{|c|}{$-26.0 \sim-50.0$} \\
\hline
\end{tabular}




\section{EXAMPLE 3: NON-CURVING AND CURVING PERPENDICULAR CROSS JOINTS FROM BELMONT, NEW YORK}

As an example of non-curving cross joints, I use the cross joints observed in the Canadaway Group in the Genesee River bed at Belmont, New York (Fig. 4-14a), reported by Engelder and Gross (1993). As described by Engelder and Gross (1993), the joints at Belmont group into three sets, which are the strike-perpendicular systematic joint set, the eastwest systematic joint set and the non-systematic joint set (Fig. 4-14b). The strike-perpendicular systematic joints are oriented $320^{\circ}$, which reflect the orientation of the maximum horizontal stress during the Alleghanian orogeny (Engelder and Gross, 1993). The east-west systematic joints are oriented $085^{\circ}$. Their origin is unknown. The non-systematic joints formed in between the strike-perpendicular systematic joints. For the purpose of maintaining consistent nomenclature, I refer to the strike-perpendicular systematic joints as pre-existing joints, and the non-systematic joints as cross joints.

As shown in Fig. 4-14b, the cross joints can be classified into two groups, (3a) and (3b). Cross joint (3a) is oriented $071^{\circ}$ and has an angle of $69^{\circ}$ with the pre-existing joints, while cross joint $(3 \mathrm{~b})$ is oriented $054^{\circ}$ with an angle of $86^{\circ}$ with the pre-existing joints. From their orientations, we can determine that the maximum horizontal stress was in the direction of $071^{\circ}$ during the formation of cross joint (3a), and was in the direction of $086^{\circ}$ during the formation of cross joint (3b). Furthermore, we can see 
(a)

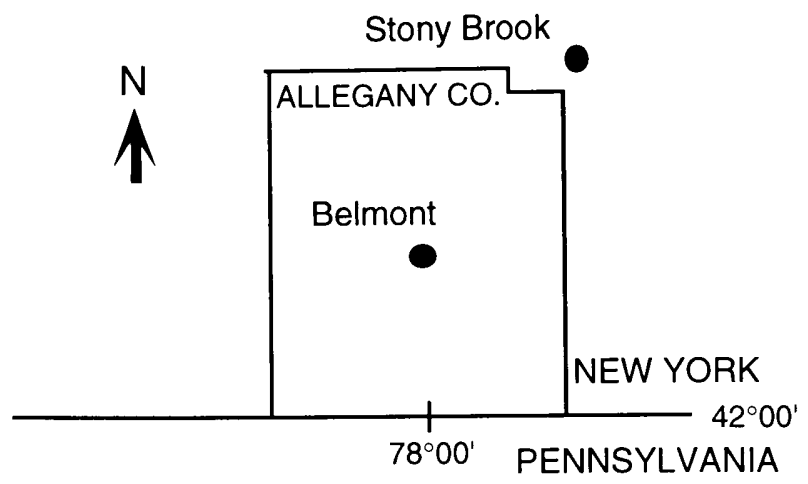

(b)

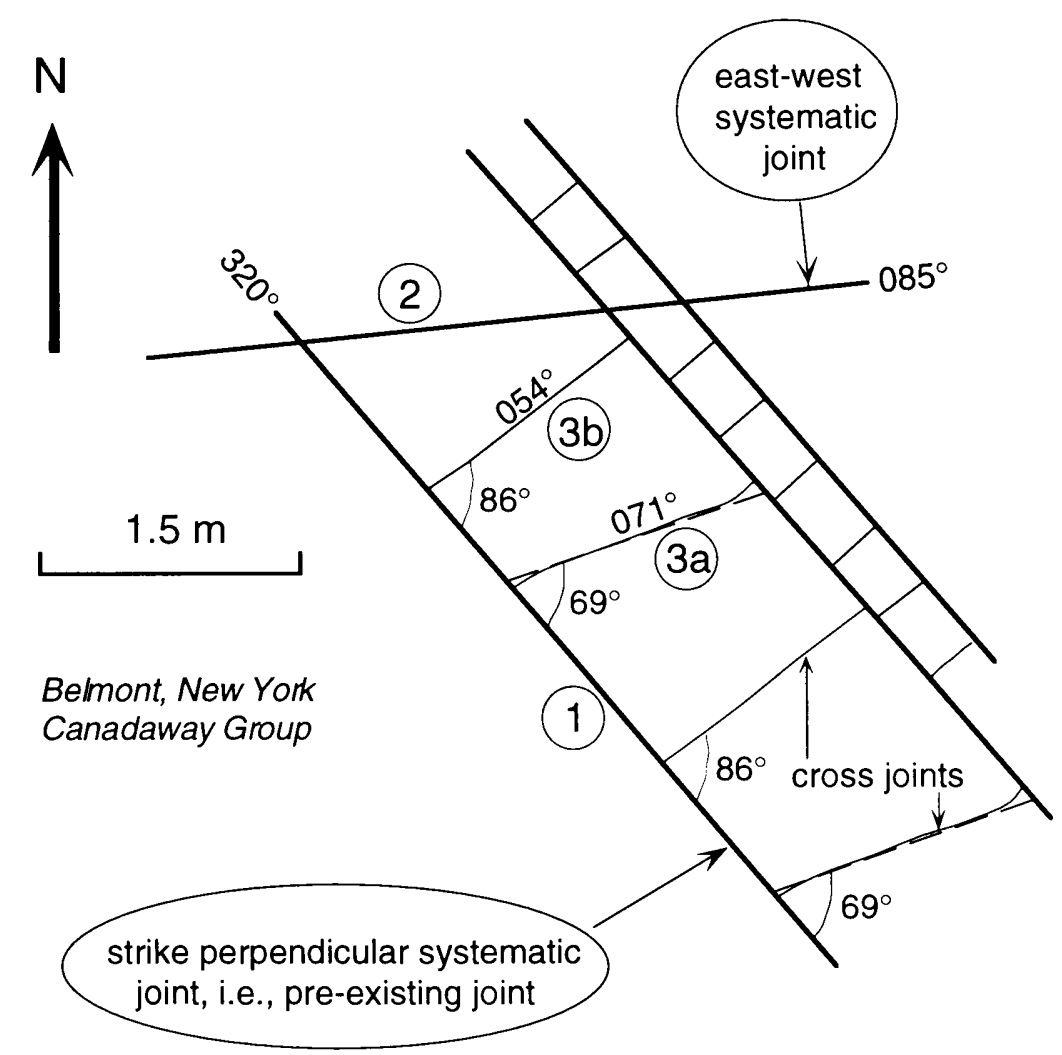

Fig. 4-14. (a) Map showing the location of Belmont, New York. (b)

Sketch diagram showing the three joint sets in Canadaway Group siltstones in Genesee River bed at Belmont, New York. The circled numbers in the figure indicate the relative ages of the joint sets from oldest (1) to youngest (3). After Engelder and Gross (1993). 
that the pre-existing joints were open during the formation of cross joint (3a) because purely curving-perpendicular geometries can only occur in the open case (Table 3-3). However, based on Table 3-3 the pre-existing joints were closed during the formation of cross joint (3b).

Now let us consider what information we can determine regarding the remote effective principal stress ratio. For the non-curving case, we can only estimate the range for the remote effective principal stress ratio. The condition for the non-curving geometry is $C_{0}<C_{0}^{*}$ as shown in Table 33. By referring to equation (3-37) in terms of the effective remote principal stress ratio $\left(\eta^{*}\right)$, we have the complete expression for this condition as

$$
C_{0}<\frac{\left(\eta^{*}-1\right) \sin 2 \varsigma}{\eta^{*}(1-\cos 2 \varsigma)+(1+\cos 2 \varsigma)},
$$

Rewriting equation (4-1), we arrive at the range for the remote effective principal stress ratio, which is

$$
\eta^{*}<-\frac{C_{0}(1+\cos 2 \varsigma)+\sin 2 \varsigma}{C_{0}(1-\cos 2 \varsigma)-\sin 2 \varsigma} .
$$

For the non-curving cross joint in Fig. 4-14b, i.e., cross joint (3b), the take off angle is $86^{\circ}$. By substituting this value into equation (4-2), we get the relation between the maximum remote effective principal stress ratio versus the coefficient of friction along the pre-existing joint ( $C_{0}$ ), which is shown in Fig. 4-15. For $C_{0}=0.6$, we have $\eta^{*}<-0.14$, which defines the 


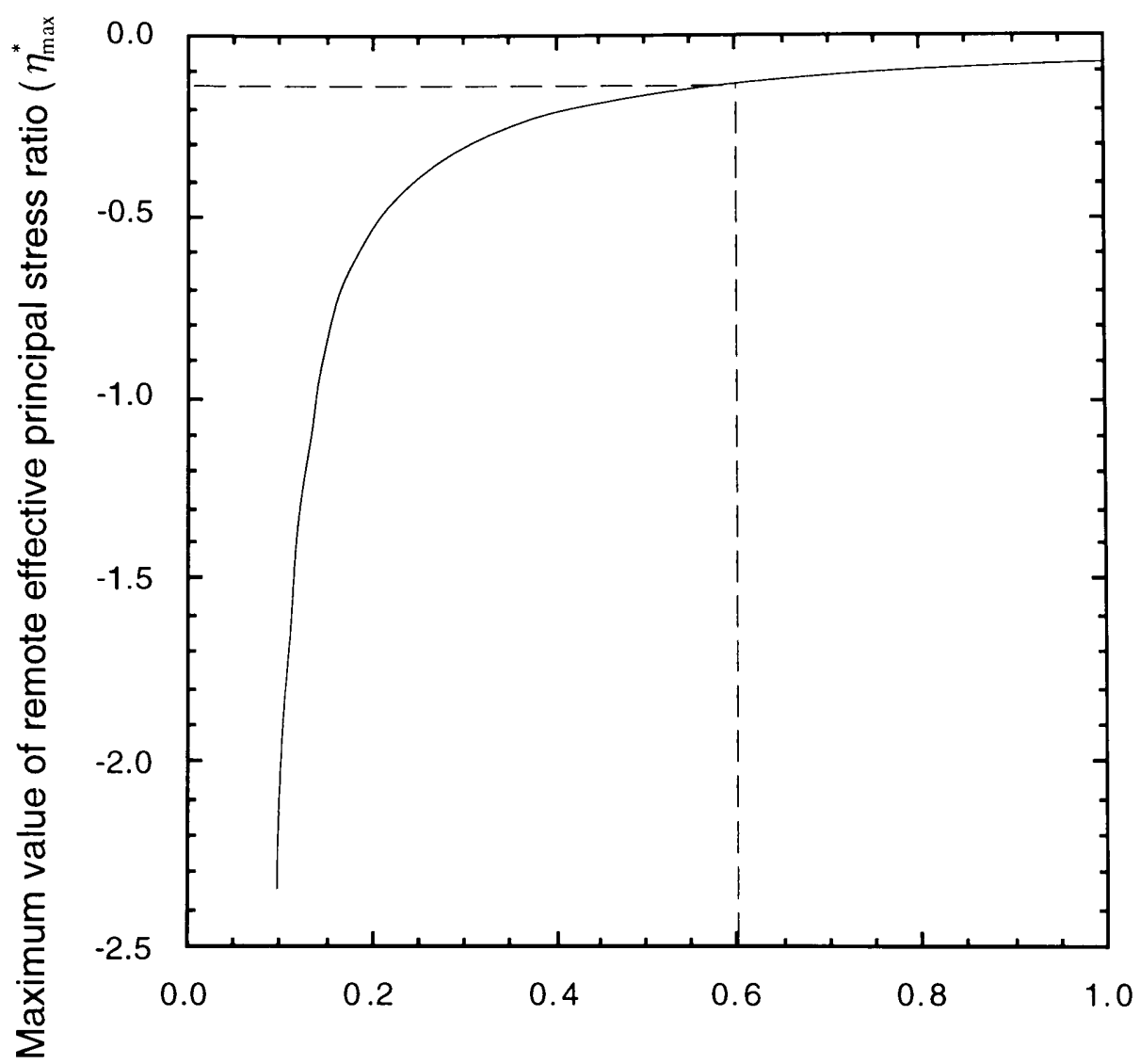

Coefficient of Friction along the Pre-existing joint $\left(\mathrm{C}_{0}\right)$

Fig. 4-15. Relation between the maximum value of remote effective principal stress ratio and the coefficient of friction along the preexisting joint for the non-curving cross joint, i.e., cross joint ( $3 b)$, in Fig. 4-10. 
Table 4-3. Remote effective principal stress and pre-existing joint information obtained from cross joint (3a) and (3b) in Figure 4-10.

\begin{tabular}{||c|c|c||}
\hline & Cross joint (3a) & Cross joint (3b) \\
\hline $\begin{array}{c}\text { Direction of the maximum } \\
\text { horizontal principal stress }\end{array}$ & $071^{\circ}$ & $054^{\circ}$ \\
\hline $\begin{array}{c}\text { Status of the pre-existing joint } \\
\text { during the formation of the } \\
\text { cross joint }\end{array}$ & open & closed \\
\hline $\begin{array}{c}\text { Range of the remote effective } \\
\text { principal stress ratio }\left(\eta^{*}\right)\end{array}$ & $-0.15<\eta^{*}<1.0$ & $\eta^{*}<-0.14$ \\
\hline
\end{tabular}


range of the remote effective principal stress ratio during the formation of cross joint (3b) (Fig. 4-15).

Cross joint (3a) shows a curving-perpendicular geometry, which belongs to the Open Case \#3 in Table 3-3. Using the equations provided in Table 3-3, we calculate that the range of the remote effective principal stress ratio during the formation of cross joint ( $3 a$ ) falls in the range of $-0.15<\eta^{*}<1.0$. It is not possible to derive an accurate remote effective principal stress ratio using the path-fit method because the bed thickness is not available. The results from the above discussion are summarized in Table 4-3.

\section{SUMMARY}

The above analyses show that our theoretical results can be useful in analyzing the remote principal stress orientations and relative stress magnitudes based on field observations of cross joint geometry. Two methods can be used to analyze the remote effective principal stress ratio, the path-fit method and the compressive zone dimension method. The path-fit method can be applied when cross joints belong to curving-parallel (Open Case \#1) (refer to Table 3-3), curving-perpendicular ( Open Cases $\# 2$ and \#3), quasi-curving-parallel (Closed Case \#1) and quasi-curvingperpendicular (Closed Cases \#2a and \#3a) categories. The compressive zone dimension method can be used in the Closed Case \#2a and \#3a, i.e., when compressive zones exist. The non-curving cases (Closed Cases \#2b 
and \#3b) can only occur when the pre-existing joint is closed. From the orientations of non-curving cross joints, we can only estimate the range of the remote effective principal stress ratio.

One noteworthy item to mention here is that the application of the theory is limited by its assumptions. In using this theory, the spacing between the pre-existing joints and between the cross joints needs to be relatively high because effects of joint interactions are not considered in our derivations. This limitation is clearly shown in discussing example 1 in this chapter. 


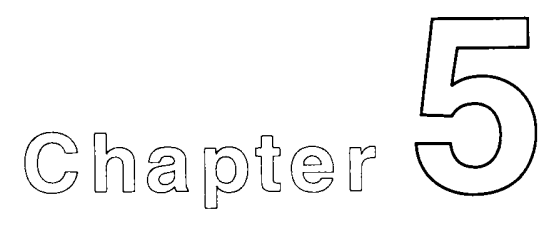

\section{DISCUSSION AND SUMMARY}

The study of the relationship between cross joint geometry and remote stress field as well as pore pressure in the previous chapters demonstrates that cross joint geometry can be used as an indicator of both the orientations and ratio of remote stresses during their formation. A detailed theoretical study based on the remote principal stress ratio, takeoff angle and the coefficient of friction along the pre-existing joint shows that cross joint geometries can vary among curving-parallel, curvingperpendicular, non-curving, quasi-curving-parallel and quasi-curvingperpendicular configurations. In some of the closed pre-existing joint cases, there exist compressive zones in the vicinity of the pre-existing joint. In a compressive environment, pore pressure plays a key role in propagating cross joints and in controlling cross joint geometry. Applying the analytical solutions to cross joints observed in the field shows that cross 
joint geometries and orientations can be used to constrain the stress state during cross joint development. Based on different cross joint geometries, two different methods, the path-fit method and the compressive zone dimension method, can be used to estimate the remote principal stress ratio.

I have attempted to build upon the advances of Dyer's (1988) work by (1) providing the general conditions for an open and a closed preexisting joint at an arbitrary take off angle $(\varsigma)$ (equations (3-4) and (3-5)); (2) developing a detailed cross joint classification. By studying the local cross joint angle ( $\theta$, i.e., the angle between cross joint along its path and the pre-existing joint), cross joint geometries can be classified as quasicurving-perpendicular, quasi-curving-parallel and non-curving in addition to Dyer's curving-parallel and curving-perpendicular geometries. For each of the above cross joint geometries, the general conditions with respect to the remote principal stress ratio at an arbitrary take off angle are provided (Table 3-3); (3) deriving a general formulation for the dimension of the compressive zone, which shows that the dimension of the compressive zone depends not only upon the take off angle and the remote principal stress ratio, but also upon the coefficient of friction along the pre-existing joint (equation (3-60)); and (4) accounting for fluid pressure in the analytical solution, which provides a method to study cross joint geometry variation in a compressive stress environment.

There are two significant aspects that result from this study. First, by detailed observation and measurement of cross joint geometry in the field, one can determine the principal stress orientations and estimate the stress ratios that prevailed during cross joint growth. Where cross joints 
are present, the regional documentation of cross joints can constrain the regional stress field, which may yield valuable information concerning the tectonic development of a region. Second, based on the stress orientations and ratios as well as the orientation of the pre-existing joint set in an area, one can predict the expected cross joint geometry. In other words, one can predict the type of cross joint geometry that would develop, for example, in the subsurface. Together with the pre-existing joints, the predicted cross joint geometry may provide a hypothetical picture of the fracture network in a region, which in turn is a main factor in controlling fluid conductivity and very important in groundwater modeling and oil and gas exploration and production.

In the next section, I will discuss the implications of cross joint geometry on fluid conductivity in more detail based on the different possible types of cross joint geometries. Following that, I will discuss the significance of the compressive zone. Then I will discuss relations between cross joint geometries and fluid pressure. In the last two sections I will analyze the limitations of the analytical solutions and propose some future work.

\section{CROSS JOINT GEOMETRY AND FLUID CONDUCTIVITY}

To study the effect of cross joint geometry on the hydraulic conductivity or conductivity for oil and gas, let us consider the following situations. Here I use the term fluid conductivity instead of hydraulic 
conductivity or conductivity for oil and gas. Assuming the fluid conductivity of the rock which contains the fluid is very low prior to developing any joints, the rock is homogeneous and isotropic in plan view (Fig. 5-1a). The coordinate system is designated as both $i$ and $j$ directions are parallel to the bedding plane (Fig. 5-1). Therefore, before any joint develops, fluid conductivity in both the $i$ and $j$ directions, i.e., $K_{i}$ and $K_{j}$, respectively, are very low. After that, a systematic joint set develops in the direction parallel to the $i$ direction (Fig. 5-1b). As a result, fluid conductivity in the $i$ direction $\left(K_{i}\right)$ increases dramatically (Fig. 5-1b) according to the theory of flow in fractures (Fetter, 1994). If we take the systematic joints as the pre-existing joints described in the previous sections, and assume there is a stress field rotation, then cross joints develop in between the systematic joint as shown in Fig. 5-1c, $d, e, f, g, h$ and $k$ Fig. 5-1c and $d$ depict compressive zones adjacent to the pre-existing joints. Because cross joints cannot propagate into a compressive zone, they cannot be connected with the pre-existing joints. As a result, fluid conductivity in the $j$ direction $\left(K_{j}\right)$ does not change. However, if compressive zones do not exist, the cross joints can be connected with the pre-existing joints, and fluid conductivity in the $j$ direction $\left(K_{j}\right)$ will increase considerably (Figs. 5-1e, $\mathrm{f}, \mathrm{g}, \mathrm{h}$ and $\mathrm{k}$ ). The relative magnitudes of $K_{j}$ in Figs. 5-1 $e, f, g, h$ and $k$ may vary according to the lengths of the cross joint paths, which are shown schematically in Fig. 5-2. 

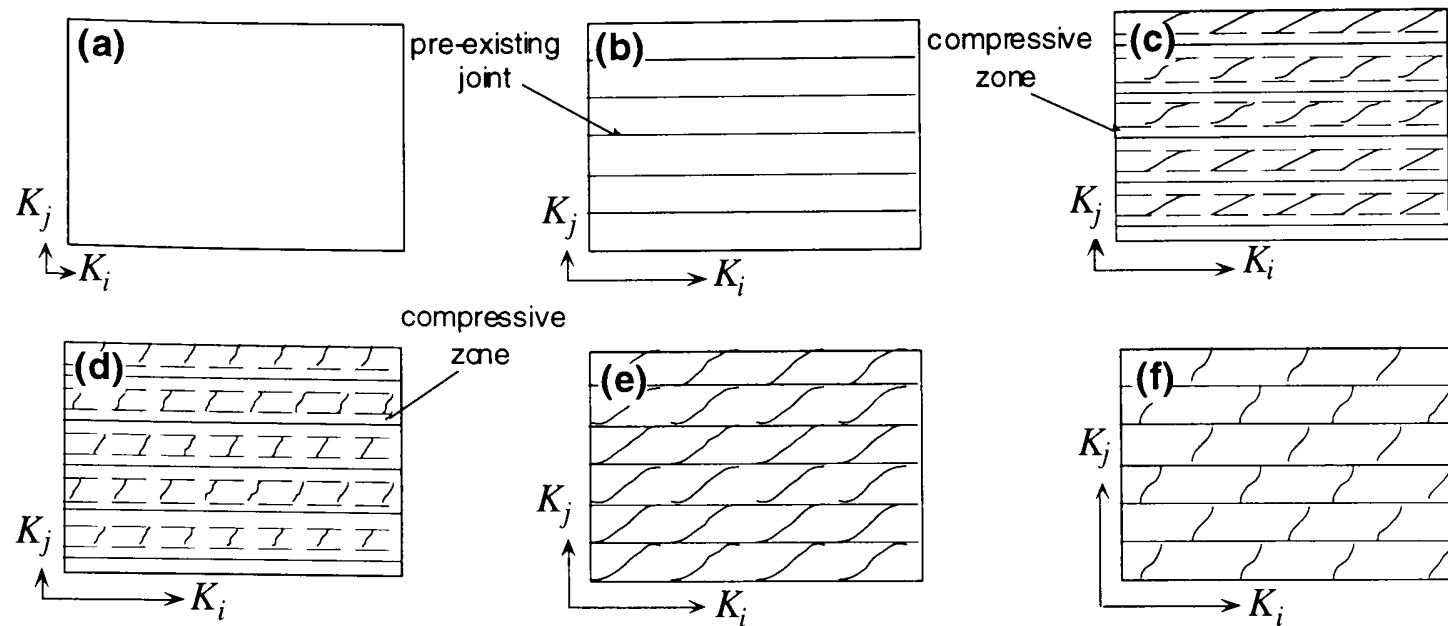

compressive
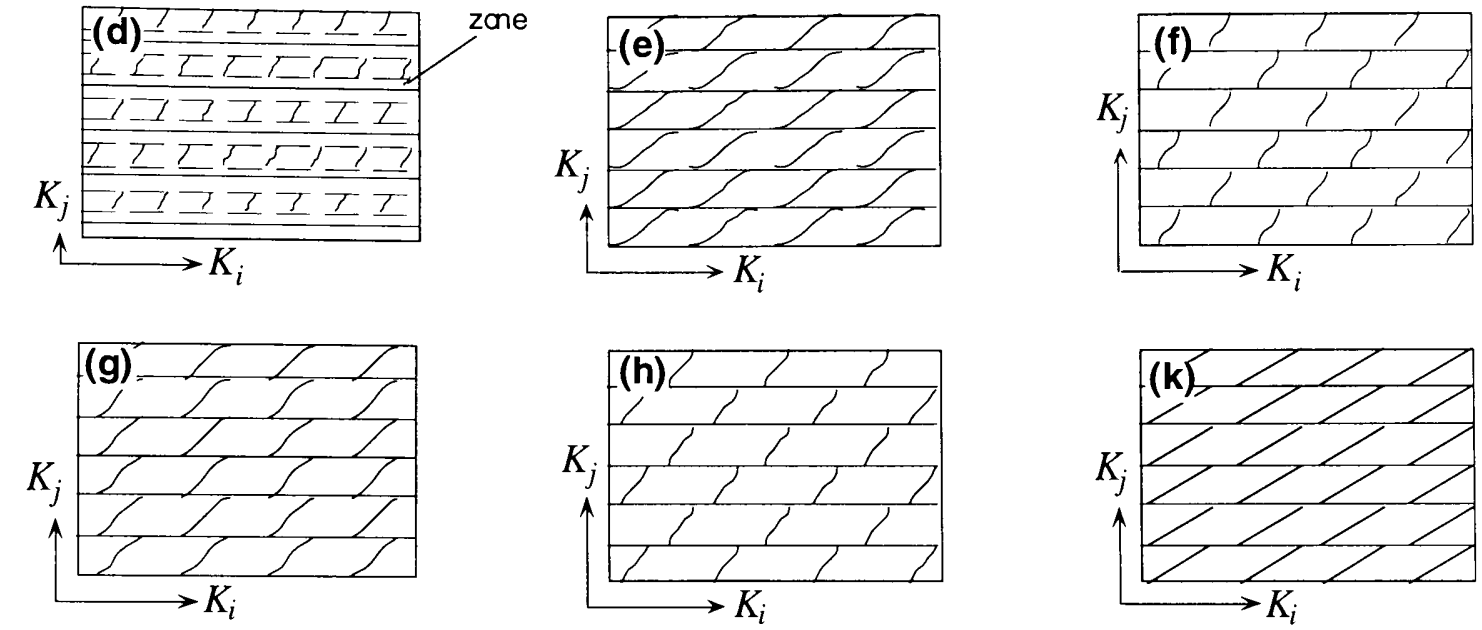

Fig. 5-1. Sketch diagrams showing the relationships between cross joint geometries and fluid conductivity of rocks. (a) No jointing.
(b) Only pre-existing joints.
(c) Pre-existing joints and quasi-

curving-parallel cross joints with compressive zones in the vicinity of pre-existing joints. (d) Pre-existing joints and quasi-curvingperpendicular cross joints with compressive zones in the vicinity of pre-existing joints. (e) Pre-existing joints and curving-parallel cross joints. (f) Pre-existing joints and curving-perpendicular cross joints. (g) Pre-existing joints and quasi-curving-parallel cross joints without compressive zones. (h) Pre-existing joints and quasi-curvingperpendicular cross joints. (k) Pre-existing joints and non-curving cross joints. The lengths of $K_{i}$ and $K_{j}$ depict the relative magnitudes of fluid conductivity in the corresponding directions. 


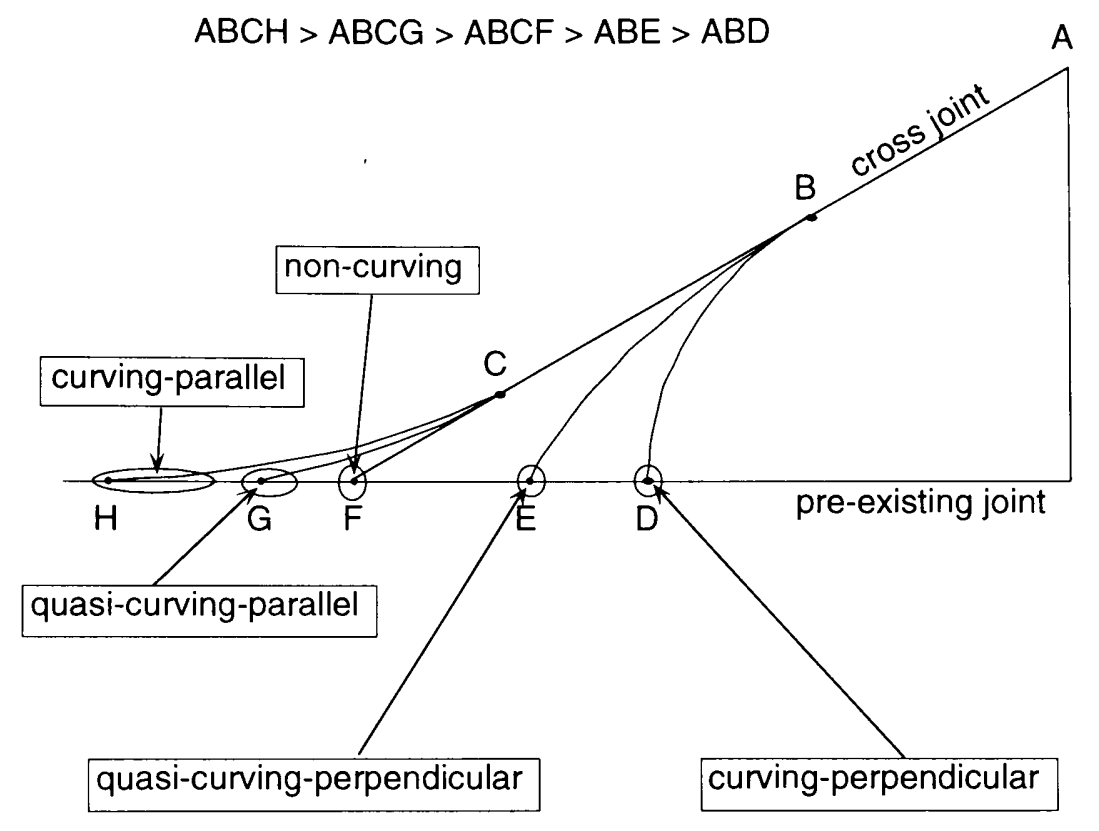

Fig. 5-2. Cross joint geometries and their relative lengths of their paths. Note that shorter paths will provide a higher conductivity. 


\section{SIGNIFICANCE OF COMPRESSIVE ZONE}

The significance of the compressive zone must be noted in two different ways. One is that the compressive zone can give information about the remote principal stress ratio. The other is that compressive zones serve as barriers for fluid flow.

As mentioned in the previous section, cross joints cannot propagate into a compressive zone where all the principal stresses are compressive. This provides the criterion for determining compressive zones in the field. Theoretically, the compressive zone lies in the vicinity of the pre-existing joint with a boundary line parallel to the pre-existing joint. In doing field work, one can determine the boundary of a compressive zone by connecting the termination points of cross joints belonging to the same cross joint set. The same cross joint set refers to cross joints that formed at approximately the same time under the same stress field. After determining the boundary of the compressive zone, one can measure the dimension of the compressive zone. Together with other measurements from the cross joints and the preexisting joint, such as orientations, one can determine the principal stress ratio during the formation of the cross joints using the compressive zone dimension method as shown in chapter 4 .

In the previous section I already discussed the implications of compressive zones on fluid conductivity. Now let us consider the following questions:

(1) How does one predict the existence of compressive zones?

(2) What kind of parameters are necessary to make this prediction? 
(3) Under what conditions are compressive zones most likely to exist? To answer these questions, let us recall the theoretical results in chapter 3.

Table 3-3 and Fig. 3-16 show the cross joint geometry classification based on the theoretical derivations. From the table and figure, we can see that in order to predict the existence of a compressive zone, one needs the take-off angle $(\varsigma)$, the remote principal stress ratio $(\eta)$, and the coefficient of friction along the pre-existing joint. The coefficient of friction along the pre-existing joint can be easily obtained from the literature (e.g. Handin, 1966) or by conducting some experimental work. In order to determine the take-off angle and remote principal stress ratio one requires the orientation of the pre-existing joints and the orientations and relative magnitudes of the principal stresses. With the knowledge of these parameters in hand, one can easily predict the category of the cross joints by using the inequalities in Table 3-3 or by diagrammatic plotting the remote principal stress ratio $(\eta)$ versus the take-off angle $(\varsigma)$ in Fig. 3-16. If the predicted cross joint geometry category is Closed Case \#1 or Closed Case \#2Ia, compressive zone exists. Otherwise, a compressive zone does not exist. The favorable conditions for the existence of a compressive zone can be seen from Fig. 3-15. Fig. 3-15a shows that the more negative the remote principal stress ratio, the more likely it is for the compressive zone to exist. Fig. 3-15b shows that compressive zones are most likely to exist when the take-off angle is a medium value $\left(\sim 40^{\circ}\right)$. 


\section{SIGNIFICANCE OF THE RELATION BETWEEN CROSS JOINT GEOMETRY AND FLUID PRESSURE}

The relation between pore pressure and cross joint geometry is a rather complicated problem, especially when the take off angle is greater than $45^{\circ}$ (Table 3-3 and Fig. 3-16). The general trend in the case of take off angle less than $45^{\circ}$ is that cross joint geometry changes from quasicurving-parallel with compressive zone to curving-parallel to curvingperpendicular with increasing pore pressure (Fig. 3-18). In this process, connectivity between cross joints and pre-existing joints is improved. The same can be said for the case when the take off angle is $50^{\circ}$ (Fig. 3-19). For the other cases described in chapter 3 (Fig. 3-20, 3-21, and 3-22), compressive zones do not exist, and the general change in cross joint trend is from non-curving to quasi-curving-perpendicular to curvingperpendicular. Therefore, on theoretical grounds all cross joints in these categories are initially connected. However, one thing that can be said is that fluid conductivity in the direction normal to the pre-existing joint is improved with increasing pore pressure because cross joint length is systematically reduced when cross joint geometry changes from noncurving to quasi-curving-perpendicular to curving-perpendicular (Fig. 52).

These results provide a tool for predicting cross joint geometry with the knowledge of remote principal stress ratio, orientations of the maximum horizontal stress and the pre-existing joints, as well as the magnitude of fluid pressure. Conversely, we can predict the magnitude of fluid pressure 
if we know the cross joint geometry, the remote principal stress ratio, and the orientations of the maximum horizontal stress and the pre-existing joints. Most interestingly, in an area where cross joint geometry changed through time (e.g., the Monterey cross joints in example 1 of chapter 4), we can garner some information about the history of fluid pressure variations in the area.

\section{LIMITATIONS IN USING THE THEORETICAL RESULTS}

In using the analytical results provided in chapter 3 , one must remember the assumptions in the theoretical derivations. The assumptions I made are (1) a cross joint path is perpendicular to the local least horizontal stress in terms of the theoretical model (Fig. 3-2), (2) there is no interaction between a growing cross joint and the pre-existing joint, and (3) there is no interaction between two cross joints. Under the limitations of these assumptions, we can only use the theoretical results when the spacing of the pre-existing joints and that of the cross joints are relatively high. In terms of fracture spacing index (FSI, i.e., the ratio of mechanical layer thickness, MLT, to median joint spacing, defined as the slope of the best-fit line on plots of MLT versus median joint spacing, with median joint spacing as the dependent variable) or fracture spacing ratio (FSR, i.e., the ratio of mechanical layer thickness to median joint spacing in a jointed layer) (Narr, 1991, Narr and Suppe, 1991, Gross, 1993a), the FSI or FSR must be relatively low. Otherwise, interactions between a growing cross joint and 
the pre-existing joint and that of adjacent cross joints must be considered. However, studying interaction between different joint sets is beyond the capacity of the analytical method. Numerically it is possible to solve this problem.

\section{FUTURE WORK}

Although the analytical work in this thesis builds upon Dyer's (1988) analysis, some further studies should be continued in the future, especially pertaining to verification of the assumptions.

As I stated in discussing the limitations of the analytical solutions, in order to satisfy the assumptions, the spacing of the pre-existing joints and that of the cross joints must be relatively high. One may ask the question "How high?" Frankly speaking, I do not know exactly what the answer is. Analytically, the only clue can be drawn from the Saint-Venant Principle (Timoshenko and Goodier, 1970). Based on the Saint-Venant Principle, we can say that the spacing of the pre-existing joints and that of the cross joints must be at least 5 to 10 times the height of the pre-existing joint. In order to get the exact answer to the question, I suggest the following work.

First, one needs to do some numerical modeling. Although it is impossible to include fracture interactions analytically, numerically it is possible to include a number of fractures in a single model. Therefore, fracture interactions can be studied using a numerical model. For the purpose of studying cross joint geometries, I suggest to use a 3-D model, 
because through fractures need to be introduced into the model to represent the pre-existing joints, and layered structures need to be used in order to represent the real situations in layered sedimentary rocks. Using a 3-D model, one can study cross joint propagation paths by actually propagating the cross joints in the model under different boundary conditions. Then one can verify the analytical solutions by comparing the modeled cross joint paths with the analytical solutions.

Second, one can verify the analytical solutions by doing some more detailed field work. For this purpose, one needs to select an area with welldeveloped cross joints where the orientations and relative magnitudes of principal stresses can be independently derived. By measuring the cross joint paths and the orientation of the pre-existing joints, one can use the analytical solutions in this thesis to obtain the orientations and ratio of the remote stress field during cross joint formation. By comparing the results of orientations and relative magnitudes of the remote principal stresses from the cross joints to those from other independent methods, one can verify the analytical solutions in this thesis.

To finish any of the above projects, much more effort needs to be devoted beyond solving for the equations in this thesis. To mark the end of this thesis, I wish to invoke a Chinese proverb. It says " No matter how long a road is, you are able to reach to its end, if you walk steadily step by step; no matter how high a mountain is, you are able to reach to its peak, if you climb steadily step by step." 


\section{REFERENCES}

Bahat, D., and Engelder, T., 1984, Surface morphology on cross-fold joints of the Appalachian Plateau, New York and Pennsylvania: Tectonophysics, v. 104, p. 293-313.

Byerlee, J. D., 1978, Friction of rock: Pure and Applied Geophysics, v. 116, p. 615-626.

Broek, D., 1986, Elementary engineering fracture mechanics, 4th edition: Martinus Nijhoff, Boston, 516p.

Cherepanov, G. P., 1979, Mechanics of brittle fractures: McGraw Hill, New York, 939p.

Cloos, H., 1922, Uber Ausbau und Anwendung der granittektonischen Methode: Preussischen Geologischen Landesanstalt, v. 89, p. 1-18.

Dunham, J. B., and Blake, G. H., 1987, Guide to coastal outcrops of the Monterey Formation of western Santa Barbara County, California: Field Trip Guide, Pacific Section SEPM, 35p.

Dyer, R., 1988, Using joint interactions to estimate paleostress ratios: Journal of Structural Geology, v. 10, p. 685-699. 
Engelder, T., 1987, Joints and shear fractures in rock: in Atkinson, B., ed., Fracture Mechanics of Rock, Academic Press, Orlando, Florida, p. 27-69.

Engelder, T., 1993, Stress Regimes in the Lithosphere: Princeton University Press, Princeton, New Jersey, 451 p.

Engelder, T., and Geiser, P., 1980, On the use of regional joint sets as trajectories of paleostress fields during the development of the Appalachian Plateau, New York: Journal of Geophysical Research, v. 85, p. 6319-6341.

Engelder, T., and Gross, M. R., 1993, Curving cross joints and the lithospheric stress field in eastern North America: Geology, v. 21, p. 817-820.

Engelder, T., and Lacazette, A., 1990, Natural hydraulic fracturing: in Barton, N., and Stephansson, O., eds., Rock Joints, Rotterdam, A. A. Balkema, p. 35-44.

Fischer, M. P., 1994, Application of linear elastic fracture mechanics to some problems of fracture propagation in rock and ice: $\mathrm{Ph} . \mathrm{D}$. thesis, Department of Geosciences, Pennsylvania State University.

Fischer, M. P., Gross, M. R., Engelder, T., and Greenfield, R. J., 1995, Finite-element analysis of the stress distribution around a pressurized crack in a layered elastic medium: implications for the spacing of 
fluid-driven joints in bedded sedimentary rock: Tectonophysics, v. 247 , p. 49-64.

Garfunkel, Z., 1978, The Neger-regional synthesis of sedimentary basins:

10th International Congress on Sedimentology, Jerusalem, Guidebook, p. 35-110.

Gross, M. R., 1993a, The origin and spacing of cross joints: examples from the Monterey Formation, Santa Barbara Coastline, California: Journal of Structural Geology, v. 15, p. 737-751.

Gross, M. R., 1993b, The effects of mechanical stratigraphy of failure mode and fracture spacing in the Monterey Formation of coastal California: Ph. D. thesis, Department of Geosciences, Pennsylvania State University.

Gross, M. R., 1995, Fracture partitioning: failure mode as a function of lithology in the Monterey Formation of coastal California: Geological Society of American Bulletin, v. 107, p. 779-792.

Gross, M. R., and Engelder, T., 1995, Curving cross joints and the lithospheric stress field in eastern North America: Geology, v. 21, p. 817-820.

Gross, M. R., Fischer, M. P., Engelder, T., and Greenfield, R. J., 1995, Factors controlling joint spacing in interbedded sedimentary rocks: integrating numerical models with field observations from the Monterey Formation, USA: in Ameen, M. S., ed. Fractography: 
fracture topography as a tool in fracture mechanics and stress analysis, Geological Society Special Publication, No. 92, p. 215-233.

Gudmundsson, A., 1983, Form and dimensions of dikes in eastern Iceland: Tectonophysics, v. 95, p. 295-304.

Hancock, P. L., 1985, Brittle microtectonics: principles and practice: Journal of Structural Geology, v. 7, p. 437-457.

Hobbs, D. W., 1967, The formation of tension joints in sedimentary rocks: an explanation: Geological Magazine, v. 104, p. 550-556.

Hodgson, R. A., 1961, Regional study of jointing in Comb Ridge-Navajo Mountain area, Arizona and Utah: American Association of Petroleum Geologists Bulletin, v. 45, p. 1-38.

Hornafius, S. J., 1985, Neogene tectonic rotation of Santa Ynez Range, Western Transverse Ranges, California, suggested by paleomagnetic investigation of the Monterey Formation: Journal of Geophysical Research, v. 90, p. 12,503-12,552.

Huang, Q., and Angelier, J., 1989, Fracture spacing and its relation to bed thickness: Geological Magazine, v. 126, p., 355-362.

Jaeger, J. C., and Cook, N. G. W., 1979, Fundamentals of rock mechanics (3rd edition): London, Chapman and Hall, 539 p. 
Johnson, R. B., 1968, Geology of the igneous rocks of the Spanish Peaks region, Colorado: U. S. Geological Survey Professional Paper 594$\mathrm{G}, 47 \mathrm{p}$.

Kulander, B. R., and Dean, S. L., 1985, Hackle plume geometry and joint propagation dynamics: in Ove Stephansson, ed., Fundamentals of Rock Joints, Sweden, Centek Publishers, p. 85-94.

Kulander, B. R., Dean, S. L., and Ward, Jr., B. T., 1990, AAPG Methods in Exploration Series, No. 8, The American Association of Petroleum Geologists, Tulsa, Oklahoma, U.S.A., 88p.

Lacazette, A., and Engelder, T., 1992, Fluid-driven cyclic propagation of a joint in the Ithaca siltstone, Appalachian Basin, New York: in Evans, B., and Wong, T., eds., Fault Mechanics and Transport Properties of Rocks, Academic Press, London, p. 297-323.

Lachenbruch, A. H., 1961, Depth and spacing of tension cracks: Journal of Geophysical Research, v. 66, p. 4273-4292.

Ladeira, F. L., and Price, N. J., 1981, Relationship between fracture spacing and bed thickness: Journal of Structural Geology, v. 3, p. 179-183.

Lawn, B. R., and Wilshaw, T. R., 1975, Fracture of brittle solids: Cambridge, Cambridge University Press, 204p.

Marshak, S., and Mitra, G., 1988, Basic methods of structural geology: Prentice Hall, Englewood Cliffs, New Jersey, 446p. 
Masuda, T., and Kuriyama, M., 1988, Successive "mid-point" fracturing during microboudinage: an estimate of the stress-strain relation during a natural deformation: Tectonophysics, v. 147, p. 171-177.

Masuda, T., Shibutani, T., Igarashi, T., and Kuriyama, M., 1989, Microboudin structure of piedmontite in quartz schists: a proposal for new indicator of relative paleodifferential stress: Tectonophysics, v. 163 , p. $169-180$.

Masuda, T., Shibutani, T., Kuriyama, M., and Igarashi, T., 1990, Development of microboudinage: an estimate of changing differential stress with increasing strain: Tectonophysics, v. 178, p. 379-387.

McQuillan, H., 1973, Small-scale fracture density in Asmari Formation of southwest Iran and its relation to bed thickness and structural setting: American Association of Petroleum Geologists Bulletin, v. 57, p. 2367-2385.

Muller, O. H., and Pollard, D. D., 1977, The stress state near Spanish Peaks, Colorado, determined from a dike pattern: Pure and Applied Geophysics, v. 115, p. 69-86.

Namson, J., and Davis, T. L., 1990, Late Cenozoic fold and thrust belt of the southern Coast Ranges and Santa Maria Basin, California: American Association of Petroleum Geologists Bulletin, v. 74, p. 467-492. 
Narr, W., and Suppe, J., 1991, Joint spacing in sedimentary rocks: Journal of Structural Geology, v. 13, p. 675-679.

Nickelsen, R. P., and Hough, V. D., 1967, Jointing in the Appalachian Plateau of Pennsylvania: Geological Society of American Bulletin, v. 78, p. 609-630.

Nur, A., and Byerlee, J. D., 1971, An exact effective stress law for elastic deformation of rock with fluids: Journal of Geophysical Research, v. 76, p. 6414-6419.

Odé, H., 1957, Mechanical analysis of the dike pattern of the Spanish Peaks area, Colorado: Geological Society of America Bulletin, v. 68, p. 567-576.

Olson, J., and Pollard, D. D., 1989, Inferring paleostress from natural fracture patterns: a new method: Geology, v. 17, p. 345-348.

Paterson, M. S., 1978, Experimental rock deformation--The brittle field: Springer-Verlag, New York, 254 p.

Pollard, D. D., and Aydin, A., 1988, Progress in understanding jointing over the past century: Geological Society of America Bulletin, v. 100, p. 1181-1204.

Pollard, D. D., and Segall, P., 1987, Theoretical displacements and stresses near fractures in rock: with applications to faults, joints, veins, dikes, and solution surfaces: in Atkinson, B. K., ed., Fracture Mechanics of Rock: Academic Press, London, p. 277-349. 
Price, N. J., 1966, Fault and joint development in brittle and semibrittle rock: Pergamon Press, Oxford.

Rawnsley, K. D., Rives, T., Petit, J.-P., Hencher, S. R., and Lumsden, A. C., 1992, Joint development in perturbed stress field near Fault: Journal of Structural Geology, v. 14, p. 939-951.

Rodgers, J., 1970, The tectonics of the Appalachians: John Wiley, New York, 271p.

Srivastava, D., and Engelder, T., 1990, Crack-propagation and pore-fluid conditions during fault bend folding in the Appalachian Valley and Ridge, Central Pennsylvania: Geological Society of America Bulletin, v. 102, p. 116-128.

Secor, D. T., Jr., 1965, Role of fluid pressure in jointing: American Journal of Science, v. 263, p. 633-646.

Segall, P., and Pollard, D. D., 1983, Joint formation in granitic rock of the Sierra Nevada: Geological Society of America Bulletin, v. 94, p. 563-575.

Suppe, J., 1985, Principles of structural geology: Prentice-Hall, Englewood Cliffs, New Jersey, 537 p.

Timoshenko, S. P., and Goodier, J. N., 1970, Theory of elasticity (3rd edition): McGraw-Hill, New York, 567 p. 
Twiss, R. J., and Moores, E. M., 1992, Structural geology: W. H. Freeman Company, New York, $532 \mathrm{p}$.

Wu, H., and Pollard, D. D., 1995, An experimental study of the relationship between joint spacing and layer thickness: Journal of Structural Geology, v. 17, p. 887-905. 


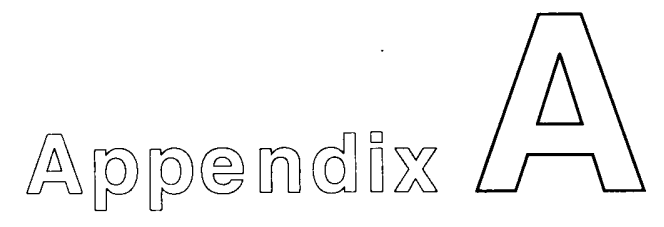

\section{HOW TO CONSTRUCT A THEORETICAL CROSS JOINT PATH}

In terms of the coordinate system defined in chapter 3 (Fig. 3-2), a cross joint path is a function of y versus z (Fig. 3-4). In chapter 3, I also provided the expressions of the local cross joint angle $(\theta)$ for the open preexisting joint case (eq. 3-24) and for the closed pre-existing joint case (eq. 3-38).

By the definition of the local cross joint angle $(\theta)$, we have

$$
\frac{\mathrm{dy}}{\mathrm{dz}}=\tan \theta \text {. }
$$

From equations (3-24) and (3-38), we can see that for a given take-off angle $(\varsigma)$ and a given remote principal stress ratio $(\eta), \theta$ is only a function of $y$, i.e., $\theta=\theta(y)$, because the half height of the pre-existing joint (c) and the 
coefficient of friction along the pre-existing joint $\left(\mathrm{C}_{0}\right)$ are constants.

Therefore, we have

$$
z=\int \frac{1}{\tan \theta} d y
$$

This equation provides the theoretical relation of $z$ versus $y$. By plotting $z$ versus $y$, we can get the theoretical cross joint path at a given take-off angle $(\varsigma)$ and a specified remote principal stress ratio $(\eta)$.

However, in reality it is not easy to plot $\mathrm{z}$ versus y according to (A2 ) because $\theta$ is a very complicated function. The cross joint paths can only be calculated numerically. The numerical approximation of equation (A-2) is

$$
\mathrm{z}_{\mathrm{n}}=\sum_{\mathrm{i}=1}^{\mathrm{n}}\left[\Delta \mathrm{y} / \tan \theta\left(\mathrm{y}_{\mathrm{i}}\right)\right]
$$

In using equation (A-3) to plot the theoretical cross joint paths in this thesis (e.g., Figs. 3-6, 4-4b), I used KaleidaGraph ${ }^{\mathrm{TM}} 3.0$ and followed the following procedures:

Step 1: Generate $y_{1}, y_{2}, \ldots, y_{m}$, with a small increment, $\Delta y$. Here $y_{1}$ is zero and $y_{m}=m \Delta y$, which is the maximum value of the distance from the pre-existing joint you want to use.

Step 2: Calculate $\Delta y / \tan \theta\left(y_{i}\right)$ for each $y_{i}(i=1,2, \ldots, m)$.

Step 3: Calculate $z_{n}$, using equation (A-3).

Step 4: Plot $y_{n}$ versus $z_{n}(n=1,2, \ldots, m)$, which gives the theoretical cross joint path. 


\section{VITA}

BORN: June 17, 1965

EDUCATION: Institute of Geology

State Seismological Bureau

Beijing, People's Republic of China

Master of Science in Geodynamics and Tectonophysics, July 1988

\section{Peking University}

Beijing, People's Republic of China

Bachelor of Science in Geology, July 1985

EXPERIENCE: Department of Geology, Florida International

University, Miami Florida

Teaching Assistant

08/94-05/96

Research Assistant

05/96-09/96

Department of Geology, Peking University

Beijing, People's Republic of China

Lecturer in Geology

1988-1994

MEMBERSHIP: Geological Society of America

Geological Society of China

Chinese Society of Rock Mechanics and Engineering 


\section{SELECTED PUBLICATIONS:}

Bai, T., Hou, J., and Wang, S., 1993, Mechanical properties of compressed rock-mud materials, Lithospheric Geosciences, Seismological Press, Beijing.

Bai, T., Hou, J., Tang, Wei, and Wang, Y., 1992, Primary study of neotectonic movement and earthquake activity in Sanmenxia Basin, Exploration of Geosciences, No.7.

Bai, T., and Wang, S., 1990, Numerical modeling of stress distribution in rock specimens under Triaxial compression with solid confining medium, Journal of Rock Mechanics and Engineering, Vol.9, No.2.

Hou, J., and Bai, T., 1993, The active feature on hanging wall of the southeastern edge listric normal fault of Wei He Basin, Shannxi Province, Earthquake Research in China, Vol.9, No.4.

Hou, J., and Bai, T., 1993, The study on geometry and kinematics in forming and evolution of extensional tectonic basins, Lithospheric Geosciences, Seismological Press, Beijing.

Hou, J., Bai., and Dong, W., 1993, The deformation, displacement and mechanism of Shabanliang new uplift in Datong Basin, Shanxi Province, Geosciences, Vol.7. No.2. 